\title{
NUMERICAL SCHEMES FOR A THREE COMPONENT CAHN-HILLIARD MODEL
}

\author{
FranCK Boyer $^{1}$ And Sebastian Minjeaud ${ }^{2}$
}

\begin{abstract}
In this article, we investigate numerical schemes for solving a three component Cahn-Hilliard model. The space discretization is performed by using a Galerkin formulation and the finite element method. Concerning the time discretization, the main difficulty is to write a scheme ensuring, at the discrete level, the decrease of the free energy and thus the stability of the method. We study three different schemes and prove existence and convergence theorems. Theoretical results are illustrated by various numerical examples showing that the new semi-implicit discretization that we propose seems to be a good compromise between robustness and accuracy.
\end{abstract}

Mathematics Subject Classification. 35K55, 65M60, 65M12, 76T30.

Received June 1, 2009. Revised July 22, 2010.

Published online December 10, 2010.

\section{INTRODUCTION}

Multiphase flows are involved in many industrial applications. For instance, in nuclear safety [31], during a hypothetical major accident in a reactor, the degradation of the core may produce multicomponent flows where interfaces undergo extreme topological changes, e.g. break-up and coalescence. Because of their ability to capture interfaces implicitly, diffuse-interface models are attractive for the numerical simulation of such phenomena. They consist in assuming that the interfaces between phases in the system have a small but positive thickness. Each phase $i$ is represented by a smooth function $c_{i}$ called the order parameter. The evolution of the system is then driven by the gradient of the total free energy, which is a sum of two terms: the bulk free energy term with a "multiple-well" shape and the capillary term depending on the gradients of the order parameters and accounting for the energy of the interfaces, that is the surface tension. For two phase situations, there has been much algorithm development and many simulations of the Cahn-Hilliard equations $[2,3,5,7,12,13,16,19,20,24]$. Generalizations of diffuse-interface models to any number of components have been recently introduced and studied as well as associated numerical methods and simulations, see for instance $[1,4,6,8,14,15,21-23,25,27,28]$.

In this article we investigate numerical schemes for solving the three component Cahn-Hilliard model fully derived and studied in [8]. We recall its main properties in Sections 1.1-1.3. One of the key features of this model

\footnotetext{
Keywords and phrases. Finite element, Cahn-Hilliard model, numerical scheme, energy estimate.

1 Université Paul Cézanne, FST Saint-Jérôme, Case cour A, LATP, Avenue Escadrille Normandie-Niemen, 13397 Marseille

Cedex 20, France. fboyer@latp.univ-mrs.fr

${ }^{2}$ Institut de Radioprotection et de Sûreté Nucléaire, Bât. 702, BP3, 13115 Saint Paul lez Durance, France.

sebastian.minjeaud@irsn.fr
} 
is a relevant choice of the bulk free energy which enables its exact coincidence with the diphasic Cahn-Hilliard model when only two phases are present in the mixture.

The space discretization is performed by using the finite element method. Concerning the time discretization, the main difficulty is to write a scheme ensuring, at the discrete level, the decrease of the free energy which is crucial to establish the existence and the convergence of approximate solutions. In some physical situations, the implicit Euler time discretization does not satisfy an energy inequality and the corresponding numerical solvers do not converge. To tackle this issue, various semi-implicit schemes are proposed and studied in Sections 2 and 3 .

We state a convergence theorem for these schemes, which enables in particular to get a proof (different from the one in [8]) of the existence of a weak solution of the Cahn-Hilliard model. Note that more general boundary conditions are taken into account here since we allow Dirichlet boundary conditions on the order parameters on some part of the boundary of the domain. Finally, in Section 5, the three schemes are numerically compared on various test cases.

\subsection{Three component Cahn-Hilliard model}

The domain $\Omega$ is an open bounded, connected, subset of $\mathbb{R}^{d}$ with $d=2$ or $d=3$. The Cahn-Hilliard approach consists in assuming that the interfaces between phases in the system have a small but positive thickness $\varepsilon$. Each phase $i$ is represented by a smooth function $c_{i}$ called the order parameter (which is taken to be the volumic fraction of the component in the mixture). The three unknowns $c_{1}, c_{2}$ and $c_{3}$ are linked though the relationship:

$$
c_{1}+c_{2}+c_{3}=1
$$

In other words, the vector $\mathbf{c}=\left(c_{1}, c_{2}, c_{3}\right)$ belongs to the hyperplane $\mathcal{S}=\left\{\left(c_{1}, c_{2}, c_{3}\right) \in \mathbb{R}^{3} ; c_{1}+c_{2}+c_{3}=1\right\}$ of $\mathbb{R}^{3}$.

The model we propose to study has been introduced in [8] (see also [10]) as a generalization of the two-phase Cahn-Hilliard model. In the diphasic case, the free energy of the mixture depends on two parameters: the interface width $\varepsilon$ and the surface tension $\sigma$. It can be written as follows:

$$
\mathcal{F}_{\sigma, \varepsilon}^{\mathrm{diph}}(c)=\int_{\Omega} 12 \frac{\sigma}{\varepsilon} c^{2}(1-c)^{2}+\frac{3}{4} \sigma \varepsilon|\nabla c|^{2} \mathrm{~d} x
$$

Therefore, in [8], the authors have postulated that the three-phase free energy can be written as follows:

$$
\mathcal{F}_{\Sigma, \varepsilon}^{\text {triph }}\left(c_{1}, c_{2}, c_{3}\right)=\int_{\Omega} \frac{12}{\varepsilon} F\left(c_{1}, c_{2}, c_{3}\right)+\frac{3}{8} \varepsilon \Sigma_{1}\left|\nabla c_{1}\right|^{2}+\frac{3}{8} \varepsilon \Sigma_{2}\left|\nabla c_{2}\right|^{2}+\frac{3}{8} \varepsilon \Sigma_{3}\left|\nabla c_{3}\right|^{2} \mathrm{~d} x .
$$

The triple of constant parameters $\boldsymbol{\Sigma}=\left(\Sigma_{1}, \Sigma_{2}, \Sigma_{3}\right)$ and the bulk energy $F$ have been determined so that the model fits with the prescribed surface tension $\sigma_{12}, \sigma_{13}$ and $\sigma_{23}$ and is "consistent" with the two-component situation (Sect. 1.2).

The evolution of the system is then driven by the gradient of the total free energy $\mathcal{F}_{\boldsymbol{\Sigma}, \varepsilon}^{\text {triph }}$ and the time evolution of $\mathbf{c}=\left(c_{1}, c_{2}, c_{3}\right)$ is governed by the following system of equations:

$$
\left\{\begin{aligned}
\frac{\partial c_{i}}{\partial t} & =\nabla \cdot\left(\frac{M_{0}(\mathbf{c})}{\Sigma_{i}} \nabla \mu_{i}\right), & & \text { for } i=1,2,3 \\
\mu_{i} & =f_{i}^{F}(\mathbf{c})-\frac{3}{4} \varepsilon \Sigma_{i} \Delta c_{i}, & & \text { for } i=1,2,3
\end{aligned}\right.
$$

where $M_{0}(\mathbf{c})$ is a diffusion coefficient called mobility which may depend on $\mathbf{c}$ and

$$
f_{i}^{F}(\mathbf{c})=\frac{4 \Sigma_{T}}{\varepsilon} \sum_{j \neq i}\left(\frac{1}{\Sigma_{j}}\left(\partial_{i} F(\mathbf{c})-\partial_{j} F(\mathbf{c})\right)\right) \text { with } \Sigma_{T} \text { defined by } \frac{3}{\Sigma_{T}}=\frac{1}{\Sigma_{1}}+\frac{1}{\Sigma_{2}}+\frac{1}{\Sigma_{3}} .
$$


This choice of $f_{i}^{F}$, obtained by the use of a Lagrange multipliers technique, enforces the condition (1.1) all along the time. Thus, one of the unknowns can be arbitrarily eliminated from the system (1.3). In Section 2.3, we will prove that we can only discretize equations satisfied by $\left(c_{1}, c_{2}, \mu_{1}, \mu_{2}\right)$ and use the relationship (1.1) to deduce $c_{3}$.

\subsection{Algebraic consistency}

At this point, it remains to specify the expression of the triple of constant parameters $\boldsymbol{\Sigma}$ and of the bulk energy $F$. These parameters have been determined so that the three phase model (defined by (1.2) and (1.3)) coincide with the diphasic model when one of the order parameters is zero. More precisely, the consistency (or algebraic consistency) of the three-phase model with the diphasic systems corresponding to one of the given surface tensions $\sigma_{12}, \sigma_{13}, \sigma_{23}$ respectively means that the following properties hold:

- When the component $i$ is not present, that is $c_{i} \equiv 0$, the total free energy $\mathcal{F}_{\boldsymbol{\Sigma}, \varepsilon}^{\text {triph }}\left(c_{1}, c_{2}, c_{3}\right)$ of the system has to be exactly equal to the total free energy $\mathcal{F}_{\sigma_{j k}, \varepsilon}^{\text {diph }}\left(c_{j}\right)$ of the diphasic system corresponding to the two other phases.

- When the component $i$ is not present in the mixture at the initial time, the component $i$ must not appear during the time evolution of the system.

It is shown in [8] that the model defined by (1.2) and (1.3) is algebraically consistent with the diphasic systems of surface tensions $\sigma_{12}, \sigma_{13}, \sigma_{23}$ respectively if and only if we have

$$
\Sigma_{i}=\sigma_{i j}+\sigma_{i k}-\sigma_{j k}, \quad \forall i \in\{1,2,3\},
$$

and there exists a smooth function $\Psi$ such that

$$
F(\mathbf{c})=\sigma_{12} c_{1}^{2} c_{2}^{2}+\sigma_{13} c_{1}^{2} c_{3}^{2}+\sigma_{23} c_{2}^{2} c_{3}^{2}+c_{1} c_{2} c_{3}\left(\Sigma_{1} c_{1}+\Sigma_{2} c_{2}+\Sigma_{3} c_{3}\right)+c_{1}^{2} c_{2}^{2} c_{3}^{2} \Psi(\mathbf{c}), \quad \forall \mathbf{c} \in \mathcal{S} .
$$

In the physical literature, the coefficient $S_{i}=-\Sigma_{i}$ defined by (1.4) is well known [30] and called the spreading coefficient of the phase $i$ at the interface between phases $j$ and $k$. If $S_{i}$ is positive (that is $\Sigma_{i}<0$ ), the spreading is said to be total and if $S_{i}$ is negative, it is said to be partial.

Notice that, in the following study, the coefficients $\Sigma_{i}$ are not assumed to be positive, so that the model presented above lets us cope with some total spreading situations (see numerical illustrations in Sects. 5.2.1 and 5.2.2). However, as shown in [8], in order for the system to be well-posed, it is needed to assume that the following condition holds:

$$
\Sigma_{1} \Sigma_{2}+\Sigma_{1} \Sigma_{3}+\Sigma_{2} \Sigma_{3}>0
$$

This condition is equivalent to the coercivity of capillary terms and consequently ensures that these terms bring a positive contribution to the total free energy. This is detailed in the following proposition.

Proposition 1.1. Let $\boldsymbol{\Sigma}=\left(\Sigma_{1}, \Sigma_{2}, \Sigma_{3}\right) \in \mathbb{R}^{3}$. There exists $\underline{\Sigma}>0$ such that, for all $n \geq 1$, for all $\left(\boldsymbol{\xi}_{\mathbf{1}}, \boldsymbol{\xi}_{\mathbf{2}}, \boldsymbol{\xi}_{\mathbf{3}}\right) \in$ $\left(\mathbb{R}^{n}\right)^{3}$ such that $\boldsymbol{\xi}_{1}+\boldsymbol{\xi}_{\mathbf{2}}+\boldsymbol{\xi}_{\mathbf{3}}=0$,

$$
\Sigma_{1}\left|\boldsymbol{\xi}_{\mathbf{1}}\right|^{2}+\Sigma_{2}\left|\boldsymbol{\xi}_{\mathbf{2}}\right|^{2}+\Sigma_{3}\left|\boldsymbol{\xi}_{\mathbf{3}}\right|^{2} \geqslant \underline{\Sigma}\left(\left|\boldsymbol{\xi}_{\mathbf{1}}\right|^{2}+\left|\boldsymbol{\xi}_{\mathbf{2}}\right|^{2}+\left|\boldsymbol{\xi}_{\mathbf{3}}\right|^{2}\right)
$$

if and only if the two following conditions are satisfied

$$
\Sigma_{1} \Sigma_{2}+\Sigma_{1} \Sigma_{3}+\Sigma_{2} \Sigma_{3}>0 \text { and } \Sigma_{i}+\Sigma_{j}>0, \forall i \neq j .
$$

This proposition and the following corollary will be useful in the sequel. In particular, under condition (1.6), Proposition 1.1 shows that the bilinear form defined by $\left(\left(\boldsymbol{\xi}_{\mathbf{1}}, \boldsymbol{\xi}_{\mathbf{2}}, \boldsymbol{\xi}_{\mathbf{3}}\right),\left(\boldsymbol{\eta}_{\mathbf{1}}, \boldsymbol{\eta}_{\mathbf{2}}, \boldsymbol{\eta}_{\mathbf{3}}\right)\right) \mapsto \sum_{i=1}^{3} \Sigma_{i} \boldsymbol{\xi}_{\boldsymbol{i}} \cdot \boldsymbol{\eta}_{\boldsymbol{i}}$ is a scalar product on $\left\{\left(\boldsymbol{\xi}_{\mathbf{1}}, \boldsymbol{\xi}_{\mathbf{2}}, \boldsymbol{\xi}_{\mathbf{3}}\right) \in\left(\mathbb{R}^{n}\right)^{3}\right.$ such that $\left.\boldsymbol{\xi}_{\mathbf{1}}+\boldsymbol{\xi}_{\mathbf{2}}+\boldsymbol{\xi}_{\mathbf{3}}=0\right\}$. The following corollary is then deduced applying the Cauchy-Schwarz inequality for this scalar product and the Young inequality. 
Corollary 1.2. Let $\boldsymbol{\Sigma}=\left(\Sigma_{1}, \Sigma_{2}, \Sigma_{3}\right) \in \mathbb{R}^{3}$ satisfying the condition (1.6). Then, for all $\left(\boldsymbol{\xi}_{\mathbf{1}}, \boldsymbol{\xi}_{\mathbf{2}}, \boldsymbol{\xi}_{\mathbf{3}}\right) \in\left(\mathbb{R}^{n}\right)^{3}$, satisfying $\boldsymbol{\xi}_{\mathbf{1}}+\boldsymbol{\xi}_{\mathbf{2}}+\boldsymbol{\xi}_{\mathbf{3}}=0$, for all $\left(\boldsymbol{\eta}_{\mathbf{1}}, \boldsymbol{\eta}_{\mathbf{2}}, \boldsymbol{\eta}_{\mathbf{3}}\right) \in\left(\mathbb{R}^{n}\right)^{3}$, satisfying $\boldsymbol{\eta}_{\mathbf{1}}+\boldsymbol{\eta}_{\mathbf{2}}+\boldsymbol{\eta}_{\mathbf{3}}=0$,

$$
\left|\sum_{i=1}^{3} \Sigma_{i} \boldsymbol{\xi}_{\boldsymbol{i}} \cdot \boldsymbol{\eta}_{\boldsymbol{i}}\right| \leqslant \frac{1}{2}\left(\sum_{i=1}^{3} \Sigma_{i}\left|\boldsymbol{\xi}_{\boldsymbol{i}}\right|^{2}+\sum_{i=1}^{3} \Sigma_{i}\left|\boldsymbol{\eta}_{\boldsymbol{i}}\right|^{2}\right) .
$$

\subsection{Existence of weak solutions}

We denote by $\Gamma$ the boundary of the domain $\Omega$ and we assume that $\Gamma$ is divided in two distinct parts $\Gamma=\Gamma_{D}^{c} \cup \Gamma_{N}^{c}$. We supplement the previous system with mixed Dirichlet-Neumann boundary conditions for each order parameter $c_{i}$ and with Neumann boundary conditions for each chemical potential $\mu_{i}$. That is, for $i=1,2$ and 3 ,

$$
\begin{aligned}
c_{i}=c_{i D} & \text { and } M_{0} \nabla \mu_{i} \cdot \mathbf{n}=0, \text { on } \Gamma_{D}^{c}, \\
\nabla c_{i} \cdot \mathbf{n}=0 & \text { and } M_{0} \nabla \mu_{i} \cdot \mathbf{n}=0, \text { on } \Gamma_{N}^{c},
\end{aligned}
$$

where $\mathbf{c}_{D}=\left(c_{1 D}, c_{2 D}, c_{3 D}\right) \in\left(\mathrm{H}^{\frac{1}{2}}(\Gamma)\right)^{3}$ is given such that $\mathbf{c}_{D}(x) \in \mathcal{S}$ for almost every $x \in \Gamma$.

Remark 1.3. The Neumann boundary condition for $\mu_{i}$ ensures in particular the conservation of the volume of the phase $i$. Indeed, we have,

$$
\frac{\mathrm{d}}{\mathrm{d} t}\left(\int_{\Omega} c_{i} \mathrm{~d} x\right)=\int_{\Gamma} \frac{1}{\Sigma_{i}}\left(-M_{0} \nabla \mu_{i}\right) \cdot \mathbf{n}=0 .
$$

The Neumann boundary conditions for $c_{i}$ impose that interfaces are normal to the boundaries of the domain and the Dirichlet boundary conditions for $c_{i}$, less classical, are used on inflow boundaries to simulate the injection of the phase $i$ (when the Cahn-Hilliard model is coupled to the Navier-Stokes equations [10]).

In view of boundary conditions (1.7)-(1.8), we introduce the following functional spaces:

$$
\begin{aligned}
\mathcal{V}^{c} & =\mathcal{V}^{\mu}=\mathrm{H}^{1}(\Omega), \\
\mathcal{V}_{D}^{c_{i}} & =\left\{\nu^{c_{i}} \in \mathrm{H}^{1}(\Omega) ; \nu^{c_{i}}=c_{i D} \text { on } \Gamma_{D}^{c}\right\}, \quad \text { for } i=1,2 \text { and } 3, \\
\mathcal{V}_{D, 0}^{c} & =\left\{\nu^{c} \in \mathrm{H}^{1}(\Omega) ; \nu^{c}=0 \text { on } \Gamma_{D}^{c}\right\}, \\
\mathcal{V}_{D, \mathcal{S}}^{\mathbf{c}} & =\left\{\mathbf{c}=\left(c_{1}, c_{2}, c_{3}\right) \in \mathcal{V}_{D}^{c_{1}} \times \mathcal{V}_{D}^{c_{2}} \times \mathcal{V}_{D}^{c_{3}} ; \mathbf{c}(x) \in \mathcal{S} \text { for a.e. } x \in \Omega\right\} .
\end{aligned}
$$

Finally, we assume that at the initial time, we have

$$
c_{i}(t=0)=c_{i}^{0},
$$

where $\mathbf{c}^{0}=\left(c_{1}^{0}, c_{2}^{0}, c_{3}^{0}\right) \in \mathcal{V}_{D, \mathcal{S}}^{\mathbf{c}}$ is given.

The existence of weak solutions of the problem (1.3) together with the initial condition (1.9) and the Neumann boundary conditions $(1.8)\left(\Gamma=\Gamma_{N}^{c}\right)$ for each unknowns $\left(c_{i}, \mu_{i}\right)$, was proved in [8] under the following general assumptions in $2 \mathrm{D}$ and $3 \mathrm{D}$ :

- The mobility $M_{0}$ is a bounded $\mathrm{C}^{1}\left(\mathbb{R}^{3}\right)$ class function and there exists three positive constants $M_{1}, M_{2}$ and $M_{3}$ such that:

$$
\begin{array}{r}
\forall \mathbf{c} \in \mathcal{S}, \quad 0<M_{1} \leqslant M_{0}(\mathbf{c}) \leqslant M_{2}, \\
\left|D M_{0}(\mathbf{c})\right| \leqslant M_{3} .
\end{array}
$$


- The bulk energy $F$ is a non negative $\mathrm{C}^{2}\left(\mathbb{R}^{3}\right)$ class function which satisfies the following polynomial growth assumptions: there exist $B_{1}>0$ and a real $p$ such that $2 \leqslant p<+\infty$ if $d=2$ or $2 \leqslant p \leqslant 6$ if $d=3$, and

$$
\begin{aligned}
\forall \mathbf{c} \in \mathcal{S}, \quad|F(\mathbf{c})| & \leqslant B_{1}\left(1+|\mathbf{c}|^{p}\right), \\
|D F(\mathbf{c})| & \leqslant B_{1}\left(1+|\mathbf{c}|^{p-1}\right), \\
\left|D^{2} F(\mathbf{c})\right| & \leqslant B_{1}\left(1+|\mathbf{c}|^{p-2}\right) .
\end{aligned}
$$

Theorem 1.4. Assume that conditions (1.5), (1.10), (1.11) hold. Consider the problem (1.3) together with the initial condition (1.9) and the Neumann boundary conditions $(1.8)\left(\Gamma=\Gamma_{N}^{c}\right)$ for each unknowns $\left(c_{i}, \mu_{i}\right)$. Then, there exists a weak solution $(\mathbf{c}, \boldsymbol{\mu})$ on $[0,+\infty[$ such that

$$
\begin{gathered}
\mathbf{c} \in \mathrm{L}^{\infty}\left(0,+\infty ;\left(\mathrm{H}^{1}(\Omega)\right)^{3}\right) \cap \mathrm{C}^{0}\left(\left[0,+\infty\left[;\left(\mathrm{L}^{q}(\Omega)\right)^{3}\right), \text { for all } q<6\right.\right. \\
\boldsymbol{\mu} \in \mathrm{L}^{2}\left(0,+\infty ;\left(\mathrm{H}^{1}(\Omega)\right)^{3}\right), \\
\mathbf{c}(t, x) \in \mathcal{S}, \text { for a.e. }(t, x) \in[0,+\infty[\times \Omega .
\end{gathered}
$$

Remark 1.5. In [8], a uniqueness theorem is also available under additional assumptions on the Hessian of the potential $F$. Notice that, in three dimensions, the proof requires a constant mobility coefficient and a slight modification of the potential $F$ that we do not consider here.

In this article we will consider Cahn-Hilliard potentials with the following form

$$
F(\mathbf{c})=\underbrace{\sigma_{12} c_{1}^{2} c_{2}^{2}+\sigma_{13} c_{1}^{2} c_{3}^{2}+\sigma_{23} c_{2}^{2} c_{3}^{2}+c_{1} c_{2} c_{3}\left(\Sigma_{1} c_{1}+\Sigma_{2} c_{2}+\Sigma_{3} c_{3}\right)}_{F_{0}(\mathbf{c})}+\underbrace{3 \Lambda c_{1}^{2} c_{2}^{2} c_{3}^{2}}_{P(\mathbf{c})} .
$$

It is important to note that in the case of partial spreading situations, i.e. $\Sigma_{i}>0, \forall i=1,2,3$, that the potential $F_{0}$ satisfies assumptions (1.11) and, consequently, the simplest choice $F=F_{0}$ is always acceptable. However, in the case of total spreading situations, i.e. one of $\Sigma_{i}$ is negative, the potential $F_{0}$ may be unbounded from below. Nevertheless, the following proposition, from [8], ensures that $F=F_{0}+P$ satisfies (1.11) provided that $\Lambda$ is large enough.

Proposition 1.6. Under condition (1.5), there exists $\Lambda_{0}>0$ such that for all $\Lambda \geqslant \Lambda_{0}$ the potential $F$ defined by (1.12) is non negative and satisfies properties (1.11).

The outline of the rest of this article is the following. In Section 2, we give the numerical scheme that we use to approximate the solution of system (1.3). The discretization of the non linear terms is stated in a general form, and we give sufficient conditions on this discretization to ensure existence of the approximate solution and its convergence towards a solution of (1.3). In Section 3, we provide several possible choices of these discretizations and we describe their main properties. In Section 4, we give the proofs of the existence and convergence theorems stated in Section 2. Note that we do not need to assume the existence of solutions of the continuous problem: we get it as a by-product of the convergence of the scheme. Hence, we provide a new proof of Theorem 1.4 considering more general boundary conditions (1.7)-(1.8). Finally, Section 5 is dedicated to some numerical experiments, in particular for the simulation of a spreading lens between two stratified other phases. The conclusion of these simulations is that the semi-implicit time discretization method we propose is a good compromise between accuracy and robustness.

\section{Discretization, existence And COnVERGEnCE of APproximate solutions}

In this section, we present the discretization of the Cahn-Hilliard system (1.3) that we will study. We first describe a semi-discretization in time in Section 2.1. Time discretization of nonlinear terms is stated in a general form; several particular possible choices will be given in Section 3. In Section 2.2, we give the space 
discretization which is performed thanks to a Galerkin approximation and the finite element method. The full discrete problem is first formulated using the three couple of unknowns $\left(c_{i h}^{n}, \mu_{i h}^{n}\right), i=1,2,3$, and then we show in Section 2.3 that this problem can be formulated using only two chosen couples of unknowns, the third one being a posteriori deduced. Finally, the rest of this section is devoted to the study of the full discrete problem. The following approach is used:

- Some a priori estimates follows from the equality of energy given in Section 2.4.

- The nonlinear discrete problem is linked by homotopy to a linear problem. The existence of an approximate solution is then deduced from the above mentioned a priori estimates and the existence of a solution of the linear problem (by applying the topological degree theory).

- The convergence of the approximate solution is obtained from the above mentioned a priori estimates by using compactness results.

Existence and convergence theorems are stated in Section 2.5. Their proofs are postponed to Section 4.

\subsection{Time discretization}

Let $N \in \mathbb{N}^{*}$ and $\left.t_{f} \in\right] 0,+\infty\left[\right.$. The time interval $\left[0, t_{f}\right]$ is uniformly discretized with a fixed time step $\Delta t=\frac{t_{f}}{N}$. For $n \in \llbracket 0, N \rrbracket$, we define $t_{n}=n \Delta t$.

Let $n \in \mathbb{N}$. We assume that functions $\left(c_{1}^{n}, c_{2}^{n}, c_{3}^{n}\right) \in \mathcal{V}_{D, \mathcal{S}}^{\mathbf{c}}$ are given. We use a semi-implicit time discretization with a special care for nonlinear terms. The scheme is written in a general way as follows, for $i=1,2,3$,

$$
\left\{\begin{aligned}
\frac{c_{i}^{n+1}-c_{i}^{n}}{\Delta t} & =\nabla \cdot\left(\frac{M_{0}^{n+\alpha}}{\Sigma_{i}} \nabla \mu_{i}^{n+1}\right), \\
\mu_{i}^{n+1} & =D_{i}^{F}\left(\mathbf{c}^{n}, \mathbf{c}^{n+1}\right)-\frac{3}{4} \varepsilon \Sigma_{i} \Delta c_{i}^{n+\beta},
\end{aligned}\right.
$$

where: • $M_{0}^{n+\alpha}=M_{0}\left((1-\alpha) \mathbf{c}^{n}+\alpha \mathbf{c}^{n+1}\right)$ with $\alpha \in[0,1]$

- $c_{i}^{n+\beta}=(1-\beta) c_{i}^{n}+\beta c_{i}^{n+1}$ with $\beta \in\left[\frac{1}{2}, 1\right]$;

- $D_{i}^{F}\left(\mathbf{a}^{n}, \mathbf{a}^{n+1}\right)=\frac{4 \Sigma_{T}}{\varepsilon} \sum_{j \neq i}\left(\frac{1}{\Sigma_{j}}\left(d_{i}^{F}\left(\mathbf{a}^{n}, \mathbf{a}^{n+1}\right)-d_{j}^{F}\left(\mathbf{a}^{n}, \mathbf{a}^{n+1}\right)\right)\right), \quad \forall\left(\mathbf{a}^{n}, \mathbf{a}^{n+1}\right) \in \mathcal{S}^{2}$.

The functions $d_{i}^{F}$ represent a semi-implicit discretization of $\partial_{c_{i}} F$. At this point, in order to ensure consistency, we only assume that

$$
D_{i}^{F}(\mathbf{c}, \mathbf{c})=f_{i}^{F}(\mathbf{c}), \forall \mathbf{c} \in \mathcal{S} .
$$

Various possible choices for these nonlinear terms will be proposed and studied in Section 3.

Following (1.7) and (1.8), the discrete boundary conditions are, for $i=1,2,3$,

$$
\begin{gathered}
c_{i}^{n+1}=c_{i D} \text { and } M_{0} \nabla \mu_{i}^{n+1} \cdot n=0, \text { on } \Gamma_{D}^{c}, \\
\nabla c_{i}^{n+1} \cdot n=0 \text { and } M_{0} \nabla \mu_{i}^{n+1} \cdot n=0, \text { on } \Gamma_{N}^{c} .
\end{gathered}
$$

\subsection{Space discretization}

For the space discretization, we use a Galerkin approximation and the finite element method. Let $\mathcal{V}_{h}^{c}$ and $\mathcal{V}_{h}^{\mu}$ be two finite element approximation subspaces of $\mathcal{V}^{c}$ and $\mathcal{V}^{\mu}$ respectively. Since order parameters verify non-homogeneous Dirichlet boundary conditions on $\Gamma_{D}^{c}$, we use $c_{i}^{0}$ as a lifting of $c_{i D}$ in $\mathcal{V}^{c}$ and we assume that functions $c_{i h}^{0} \in \mathcal{V}_{h}^{c}$ are given for all $i \in\{1,2,3\}$, for all $h>0$ such that

$$
\mathbf{c}_{h}^{0}(x) \in \mathcal{S}, \forall h>0 \text {, a.e. } x \in \Omega \text { and }\left|\mathbf{c}_{h}^{0}-\mathbf{c}^{0}\right|_{\left(\mathrm{H}^{1}(\Omega)\right)^{3}} \underset{h \rightarrow 0}{\longrightarrow} 0 .
$$


These functions $c_{i h}^{0}$ can be obtained from $c_{i}^{0}$ by $\mathrm{H}^{1}(\Omega)$-projection or, as this is the case in practice, by finite element interpolation provided that $c_{i}^{0}$ is smooth enough. We then define the following spaces:

$$
\begin{aligned}
& \mathcal{V}_{D h, 0}^{c}=\left\{\nu_{h}^{c} \in \mathcal{V}_{h}^{c} ; \nu_{h}^{c}=0 \text { on } \Gamma_{D}^{c}\right\}, \\
& \mathcal{V}_{D h}^{c_{i}}=c_{i h}^{0}+\mathcal{V}_{D h, 0}^{c}, \\
& \mathcal{V}_{D h, \mathcal{S}}^{\mathrm{c}}=\left\{\mathbf{c}_{h}=\left(c_{1 h}, c_{2 h}, c_{3 h}\right) \in \mathcal{V}_{D h}^{c_{1}} \times \mathcal{V}_{D h}^{c_{2}} \times \mathcal{V}_{D h}^{c_{3}} ; \mathbf{c}_{h}(x) \in \mathcal{S} \text { for a.e. } x \in \Omega\right\} .
\end{aligned}
$$

The general assumptions concerning the approximation spaces that we need are the following:

- $1 \in \mathcal{V}_{h}^{c} \quad$ and $\quad 1 \in \mathcal{V}_{h}^{\mu}$;

- $\forall \nu^{\mu} \in \mathcal{V}^{\mu}, \inf _{\nu_{h}^{\mu} \in \mathcal{V}_{h}^{\mu}}\left|\nu^{\mu}-\nu_{h}^{\mu}\right|_{\mathrm{H}^{1}(\Omega)} \underset{h \rightarrow 0}{\longrightarrow} 0 \quad$ and $\quad \forall \nu^{c} \in \mathcal{V}_{D, 0}^{c}, \inf _{\nu_{h}^{c} \in \mathcal{V}_{D h, 0}^{c}}\left|\nu^{c}-\nu_{h}^{c}\right|_{\mathrm{H}^{1}(\Omega)} \underset{h \rightarrow 0}{\longrightarrow} 0$;

- there exists a positive constant $C$ independent of $h$ such that:

$$
\forall \nu^{\mu} \in \mathcal{V}^{\mu},\left|\Pi_{0}^{\mathcal{V}_{h}^{\mu}}\left(\nu^{\mu}\right)\right|_{\mathrm{H}^{1}(\Omega)} \leqslant C\left|\nu^{\mu}\right|_{\mathrm{H}^{1}(\Omega)},
$$

where $\Pi_{0}^{\mathcal{V}_{h}^{\mu}}$ denote the $\mathrm{L}^{2}(\Omega)$-projection on $\mathcal{V}_{h}^{\mu}$;

- $\mathcal{V}_{h}^{c} \subset \mathcal{V}_{h}^{\mu}$.

Remark 2.1. Assumption (2.6) is available, for instance, for a family of quasi-uniform triangulations and the corresponding conforming Lagrange finite element approximation spaces [17], p. 72, (1.117).

We assume that $\mathbf{c}_{h}^{n} \in \mathcal{V}_{D h, \mathcal{S}}^{\mathbf{c}}$ is given and the Galerkin approximation of problem (2.1) at time $t_{n+1}$ is written as follows:

Problem 2.2 (formulation with three order parameters). Find $\left(\mathbf{c}_{h}^{n+1}, \boldsymbol{\mu}_{h}^{n+1}\right) \in \mathcal{V}_{D h}^{c_{1}} \times \mathcal{V}_{D h}^{c_{2}} \times \mathcal{V}_{D h}^{c_{3}} \times\left(\mathcal{V}_{h}^{\mu}\right)^{3}$ such that $\forall \nu_{h}^{c} \in \mathcal{V}_{D h, 0}^{c}, \forall \nu_{h}^{\mu} \in \mathcal{V}_{h}^{\mu}$, we have, for $i=1,2,3$,

$$
\left\{\begin{aligned}
\int_{\Omega} \frac{c_{i h}^{n+1}-c_{i h}^{n}}{\Delta t} \nu_{h}^{\mu} \mathrm{d} x & =-\int_{\Omega} \frac{M_{0 h}^{n+\alpha}}{\Sigma_{i}} \nabla \mu_{i h}^{n+1} \cdot \nabla \nu_{h}^{\mu} \mathrm{d} x, \\
\int_{\Omega} \mu_{i h}^{n+1} \nu_{h}^{c} \mathrm{~d} x & =\int_{\Omega} D_{i}^{F}\left(\mathbf{c}_{h}^{n}, \mathbf{c}_{h}^{n+1}\right) \nu_{h}^{c} \mathrm{~d} x+\int_{\Omega} \frac{3}{4} \Sigma_{i} \varepsilon \nabla c_{i h}^{n+\beta} \cdot \nabla \nu_{h}^{c} \mathrm{~d} x,
\end{aligned}\right.
$$

where $M_{0 h}^{n+\alpha}=M_{0}\left((1-\alpha) \mathbf{c}_{h}^{n}+\alpha \mathbf{c}_{h}^{n+1}\right)$ and $c_{i h}^{n+\beta}=(1-\beta) c_{i h}^{n}+\beta c_{i h}^{n+1}$.

Note that we do not seek $\mathbf{c}_{h}^{n+1}$ in $\mathcal{V}_{D h, \mathcal{S}}^{\mathrm{c}}$. The constraint $c_{1 h}^{n+1}+c_{2 h}^{n+1}+c_{3 h}^{n+1}=1$ is imposed thanks to the particular form of $D_{i}^{F}$ in the model (see Thm. 2.6).

Remark 2.3. Assumption (2.4) allows to take $\nu_{h}^{\mu} \equiv 1$ in the first equation of (2.8). This yields the exact conservation of the volume of the phases at the discrete level:

$$
\int_{\Omega} c_{i h}^{n+1} \mathrm{~d} x=\int_{\Omega} c_{i h}^{n} \mathrm{~d} x, \quad \forall i \in\{1,2,3\}, \quad \forall n \in \llbracket 0, N-1 \rrbracket .
$$




\subsection{Equivalence with a system of two coupled equations}

In practice, only the two coupled Cahn-Hilliard equations satisfied by $\left(c_{1}, c_{2}, \mu_{1}, \mu_{2}\right)$ have to be solved. Indeed, Problem 2.2 is equivalent to the following one:

Problem 2.4 (formulation with two order parameters). Find $\left(c_{1 h}^{n+1}, c_{2 h}^{n+1}, \mu_{1 h}^{n+1}, \mu_{2 h}^{n+1}\right) \in \mathcal{V}_{D h}^{c_{1}} \times \mathcal{V}_{D h}^{c_{2}} \times\left(\mathcal{V}_{h}^{\mu}\right)^{2}$ such that $\forall \nu_{h}^{c} \in \mathcal{V}_{D h, 0}^{c}, \forall \nu_{h}^{\mu} \in \mathcal{V}_{h}^{\mu}$, we have, for $i=1$ and 2 ,

$$
\left\{\begin{array}{l}
\int_{\Omega} \frac{c_{i h}^{n+1}-c_{i h}^{n}}{\Delta t} \nu_{h}^{\mu} \mathrm{d} x=-\int_{\Omega} \frac{M_{0 h}^{n+\alpha}}{\Sigma_{i}} \nabla \mu_{i h}^{n+1} \cdot \nabla \nu_{h}^{\mu} \mathrm{d} x \\
\int_{\Omega} \mu_{i h}^{n+1} \nu_{h}^{c} \mathrm{~d} x=\int_{\Omega} D_{i}^{F}\left(\mathbf{c}_{h}^{n}, \mathbf{c}_{h}^{n+1}\right) \nu_{h}^{c} \mathrm{~d} x+\int_{\Omega} \frac{3}{4} \Sigma_{i} \varepsilon \nabla c_{i h}^{n+\beta} \cdot \nabla \nu_{h}^{c} \mathrm{~d} x
\end{array}\right.
$$

with $\mathbf{c}_{h}^{n+1}=\left(c_{1 h}^{n+1}, c_{2 h}^{n+1}, 1-c_{1 h}^{n+1}-c_{2 h}^{n+1}\right)$.

Then, it remains to define

$$
c_{3 h}^{n+1}=1-c_{1 h}^{n+1}-c_{2 h}^{n+1} \quad \text { and } \quad \mu_{3 h}^{n+1}=-\left(\frac{\Sigma_{3}}{\Sigma_{1}} \mu_{1 h}^{n+1}+\frac{\Sigma_{3}}{\Sigma_{2}} \mu_{2 h}^{n+1}\right) .
$$

Remark 2.5. Notice that until the end of this article, in the systems where only the unknowns $\left(c_{1 h}^{n+1}, \mu_{1 h}^{n+1}\right.$, $\left.c_{2 h}^{n+1}, \mu_{2 h}^{n+1}\right)$ appear, the notation $\mathbf{c}_{h}^{n+1}$ represents the vector $\left(c_{1 h}^{n+1}, c_{2 h}^{n+1}, 1-c_{1 h}^{n+1}-c_{2 h}^{n+1}\right)$.

Theorem 2.6. Problem (2.8) is equivalent to problem (2.10)-(2.11). In particular, notice that any solution $\left(\mathbf{c}_{h}^{n+1}, \boldsymbol{\mu}_{h}^{n+1}\right)$ of Problem 2.2 satisfies

$$
\sum_{i=1}^{3} c_{i h}^{n+1}=1 \quad \text { and } \quad \sum_{i=1}^{3} \frac{\mu_{i h}^{n+1}}{\Sigma_{i}}=0
$$

Proof. First, by using definition (2.2) and after a reordering of terms, we find ( $j$ and $k$ are the two indices different from $i$ ):

$$
\sum_{i=1}^{3} \frac{1}{\Sigma_{i}} D_{i}^{F}\left(\mathbf{c}_{h}^{n}, \mathbf{c}_{h}^{n+1}\right)=\frac{4 \Sigma_{T}}{\varepsilon} \sum_{i=1}^{3}\left(\frac{1}{\Sigma_{i}}\left(\frac{1}{\Sigma_{j}}+\frac{1}{\Sigma_{k}}\right)-\frac{1}{\Sigma_{i} \Sigma_{j}}-\frac{1}{\Sigma_{i} \Sigma_{k}}\right) d_{i}^{F}\left(\mathbf{c}_{h}^{n}, \mathbf{c}_{h}^{n+1}\right)=0 .
$$

Assume now that problem (2.10)-(2.11) is satisfied. Then, adding equations of (2.10) for $i=1,2$ and using (2.11) and (2.13) yields to

$$
\left\{\begin{aligned}
\int_{\Omega} \frac{\left(1-c_{3 h}^{n+1}\right)-\left(1-c_{3 h}^{n}\right)}{\Delta t} \nu_{h}^{\mu} \mathrm{d} x & =-\int_{\Omega} M_{0 h}^{n+\alpha} \nabla\left(-\frac{\mu_{3 h}^{n+1}}{\Sigma_{3}}\right) \cdot \nabla \nu_{h}^{\mu} \mathrm{d} x \\
\int_{\Omega}\left(-\frac{\mu_{3 h}^{n+1}}{\Sigma_{3}}\right) \nu_{h}^{c} \mathrm{~d} x & =\int_{\Omega}\left(-\frac{1}{\Sigma_{3}} D_{3}\left(\mathbf{c}_{h}^{n}, \mathbf{c}_{h}^{n+1}\right)\right) \nu_{h}^{c} \mathrm{~d} x+\frac{3}{4} \varepsilon \int_{\Omega} \nabla\left(1-c_{3 h}^{n+\beta}\right) \cdot \nabla \nu_{h}^{c} \mathrm{~d} x .
\end{aligned}\right.
$$

This proves that $c_{3 h}^{n+1}$ satisfies (2.8) for $i=3$.

Conversely, if we assume that (2.8) is satisfied, then by adding the equations for $i=1,2,3$, thanks to (2.13), we get

$$
\left\{\begin{aligned}
\int_{\Omega} \frac{S_{h}^{n+1}-S_{h}^{n}}{\Delta t} \nu_{h}^{\mu} \mathrm{d} x & =-\int_{\Omega} M_{0 h}^{n+\alpha} \nabla \Theta_{h}^{n+1} \cdot \nabla \nu_{h}^{\mu} \mathrm{d} x \\
\int_{\Omega} \Theta_{h}^{n+1} \nu_{h}^{c} \mathrm{~d} x & =\frac{3}{4} \varepsilon \int_{\Omega}\left[(1-\beta) \nabla S_{h}^{n}+\beta \nabla S_{h}^{n+1}\right] \cdot \nabla \nu_{h}^{c} \mathrm{~d} x,
\end{aligned}\right.
$$


where $S_{h}^{\ell}=\sum_{i=1}^{3} c_{i h}^{\ell}$ and $\Theta_{h}^{\ell}=\sum_{i=1}^{3} \frac{\mu_{i h}^{\ell}}{\Sigma_{i}}$ for $\ell=n$ and $\ell=n+1$. These equations are satisfied for all $\nu_{h}^{\mu} \in \mathcal{V}_{h}^{\mu}$ and for all $\nu_{h}^{c} \in \mathcal{V}_{D h, 0}^{c}$. In particular, we take $\nu_{h}^{\mu}=\Theta_{h}^{n+1}$ and $\nu_{h}^{c}=\frac{S_{h}^{n+1}-S_{h}^{n}}{\Delta t} \in \mathcal{V}_{D h, 0}$, and get the equality

$$
\frac{3}{8} \varepsilon \int_{\Omega}\left(\left|\nabla S_{h}^{n+1}\right|^{2}-\left|\nabla S_{h}^{n}\right|^{2}+(2 \beta-1)\left|\nabla S_{h}^{n+1}-\nabla S_{h}^{n}\right|^{2}\right) \mathrm{d} x+\Delta t \int_{\Omega} M_{0 h}^{n+\alpha}\left|\nabla \Theta_{h}^{n+1}\right|^{2} \mathrm{~d} x=0 .
$$

Since $S_{h}^{n} \equiv 1\left(\mathbf{c}_{h}^{n} \in \mathcal{V}_{D h, \mathcal{S}}\right), M_{0}$ is positive and $\beta \geqslant \frac{1}{2}$, the left hand side of $(2.15)$ is a sum of non negative terms. In particular, $\nabla S_{h}^{n+1} \equiv 0$ and $\nabla \Theta_{h}^{n+1} \equiv 0$. Hence, the functions $S_{h}^{n+1}$ and $\Theta_{h}^{n+1}$ are constant. By putting these constants in the equations of $(2.14)$, we get $S_{h}^{n+1} \equiv 1$ and $\Theta_{h}^{n+1} \equiv 0$. Hence, the couple $\left(\mathbf{c}_{h}^{n+1}, \boldsymbol{\mu}_{h}^{n+1}\right)$ satisfies (2.12) and then the system (2.10)-(2.11).

\subsection{Discrete energy estimate}

The general energy estimate for our problem is obtained by a calculation similar to the one used to prove the equivalence between Problems 2.2 and 2.4 in the proof of Theorem 2.6 (see [26]).

Proposition 2.7 (discrete energy equality). Let $\mathbf{c}_{h}^{n} \in \mathcal{V}_{D h, \mathcal{S}}^{\mathbf{c}}$. Assume that there exists a solution $\left(\mathbf{c}_{h}^{n+1}, \boldsymbol{\mu}_{h}^{n+1}\right)$ of Problem 2.2. Then, the following equality holds:

$$
\begin{array}{r}
\mathcal{F}_{\mathbf{\Sigma}, \varepsilon}^{\mathrm{triph}}\left(\mathbf{c}_{h}^{n+1}\right)-\mathcal{F}_{\mathbf{\Sigma}, \varepsilon}^{\mathrm{triph}}\left(\mathbf{c}_{h}^{n}\right)+\Delta t \sum_{i=1}^{3} \int_{\Omega} \frac{M_{0 h}^{n+\alpha}}{\Sigma_{i}}\left|\nabla \mu_{i h}^{n+1}\right|^{2} \mathrm{~d} x+\frac{3}{8}(2 \beta-1) \varepsilon \int_{\Omega} \sum_{i=1}^{3} \Sigma_{i}\left|\nabla c_{i h}^{n+1}-\nabla c_{i h}^{n}\right|^{2} \mathrm{~d} x \\
=\frac{12}{\varepsilon} \int_{\Omega}\left[F\left(\mathbf{c}_{h}^{n+1}\right)-F\left(\mathbf{c}_{h}^{n}\right)-\mathbf{d}^{F}\left(\mathbf{c}_{h}^{n}, \mathbf{c}_{h}^{n+1}\right) \cdot\left(\mathbf{c}_{h}^{n+1}-\mathbf{c}_{h}^{n}\right)\right] \mathrm{d} x
\end{array}
$$

where $\mathbf{d}^{F}(\cdot, \cdot)$ is the vector $\left(d_{i}^{F}(\cdot, \cdot)\right)_{i=1,2,3}$.

Proof. On the one hand, using definition (1.2), we have

$$
\mathcal{F}_{\boldsymbol{\Sigma}, \varepsilon}^{\operatorname{triph}}\left(\mathbf{c}_{h}^{n+1}\right)-\mathcal{F}_{\boldsymbol{\Sigma}, \varepsilon}^{\operatorname{triph}}\left(\mathbf{c}_{h}^{n}\right)=\int_{\Omega} \frac{12}{\varepsilon}\left(F\left(\mathbf{c}_{h}^{n+1}\right)-F\left(\mathbf{c}_{h}^{n}\right)\right) \mathrm{d} x+\int_{\Omega} \sum_{i=1}^{3} \frac{3}{8} \Sigma_{i} \varepsilon\left(\left|\nabla c_{i h}^{n+1}\right|^{2}-\left|\nabla c_{i h}^{n}\right|^{2}\right) \mathrm{d} x
$$

On the other hand, taking $\nu_{h}^{\mu}=\mu_{i h}^{n+1}$ and $\nu_{h}^{c}=\frac{c_{i h}^{n+1}-c_{i h}^{n}}{\Delta t}$ in $(2.8)$, we get for $i=1,2,3$,

$$
\left\{\begin{aligned}
\int_{\Omega} \frac{c_{i h}^{n+1}-c_{i h}^{n}}{\Delta t} \mu_{i h}^{n+1} \mathrm{~d} x= & -\int_{\Omega} \frac{M_{0 h}^{n+\alpha}}{\Sigma_{i}}\left|\nabla \mu_{i h}^{n+1}\right|^{2} \mathrm{~d} x \\
\int_{\Omega} \mu_{i h}^{n+1} \frac{c_{i h}^{n+1}-c_{i h}^{n}}{\Delta t} \mathrm{~d} x= & \int_{\Omega} \frac{4 \Sigma_{T}}{\varepsilon} \sum_{j \neq i}\left(\frac{1}{\Sigma_{j}}\left(d_{i}^{F}\left(\mathbf{c}_{h}^{n}, \mathbf{c}_{h}^{n+1}\right)-d_{j}^{F}\left(\mathbf{c}_{h}^{n}, \mathbf{c}_{h}^{n+1}\right)\right)\right) \frac{c_{i h}^{n+1}-c_{i h}^{n}}{\Delta t} \mathrm{~d} x \\
& +\int_{\Omega} \frac{3}{4} \Sigma_{i} \varepsilon \nabla c_{i h}^{n+\beta} \cdot \nabla\left(\frac{c_{i h}^{n+1}-c_{i h}^{n}}{\Delta t}\right) \mathrm{d} x .
\end{aligned}\right.
$$

Recall that $c_{i h}^{n+\beta}=(1-\beta) c_{i h}^{n}+\beta c_{i h}^{n+1}$, so that we have

$$
\nabla c_{i h}^{n+\beta} \cdot \nabla\left(c_{i h}^{n+1}-c_{i h}^{n}\right)=\frac{1}{2}\left(\left|\nabla c_{i h}^{n+1}\right|^{2}-\left|\nabla c_{i h}^{n}\right|^{2}+(2 \beta-1)\left|\nabla c_{i h}^{n+1}-\nabla c_{i h}^{n}\right|^{2}\right) .
$$


By reordering the terms and using $\sum_{i=1}^{3}\left(c_{i h}^{n+1}-c_{i h}^{n}\right)=0$ (Thm. 2.6), we also obtain

$$
\sum_{i=1}^{3} \sum_{j \neq i}\left(\frac{1}{\Sigma_{j}}\left(d_{i}^{F}\left(\mathbf{c}_{h}^{n}, \mathbf{c}_{h}^{n+1}\right)-d_{j}^{F}\left(\mathbf{c}_{h}^{n}, \mathbf{c}_{h}^{n+1}\right)\right)\right)\left(c_{i h}^{n+1}-c_{i h}^{n}\right)=\frac{3}{\Sigma_{T}} \sum_{i=1}^{3}\left(c_{i h}^{n+1}-c_{i h}^{n}\right) d_{i}^{F}\left(\mathbf{c}_{h}^{n}, \mathbf{c}_{h}^{n+1}\right) .
$$

Hence, we deduce from (2.18) that

$$
\begin{aligned}
\Delta t \sum_{i=1}^{3} \int_{\Omega} \frac{M_{0 h}^{n+\alpha}}{\Sigma_{i}}\left|\nabla \mu_{i h}^{n+1}\right|^{2} \mathrm{~d} x= & -\frac{12}{\varepsilon} \int_{\Omega} \sum_{i=1}^{3} d_{i}^{F}\left(\mathbf{c}_{h}^{n}, \mathbf{c}_{h}^{n+1}\right)\left(c_{i h}^{n+1}-c_{i h}^{n}\right) \mathrm{d} x \\
& -\frac{3}{8} \varepsilon \int_{\Omega} \sum_{i=1}^{3} \Sigma_{i}\left(\left|\nabla c_{i h}^{n+1}\right|^{2}-\left|\nabla c_{i h}^{n}\right|^{2}\right) \mathrm{d} x \\
& -\frac{3}{8}(2 \beta-1) \varepsilon \int_{\Omega} \sum_{i=1}^{3} \Sigma_{i}\left|\nabla c_{i h}^{n+1}-\nabla c_{i h}^{n}\right|^{2} \mathrm{~d} x .
\end{aligned}
$$

The claim follows by adding (2.17) and (2.19).

Remark 2.8. Even though the $\Sigma_{i}$ are not necessarily positive, the two terms $\sum_{i=1}^{3} \Sigma_{i}\left|\nabla c_{i h}^{n+1}-\nabla c_{i h}^{n}\right|^{2}$ and $\sum_{i=1}^{3} \frac{\left|\nabla \mu_{i h}^{n+1}\right|^{2}}{\Sigma_{i}}$, involved in the left-hand side of equation (2.16), are non negative when condition (1.5) holds. Indeed, in this case, Proposition 1.1 shows that

$$
\sum_{i=1}^{3} \frac{\left|\nabla \mu_{i h}^{n+1}\right|^{2}}{\Sigma_{i}}=\sum_{i=1}^{3} \Sigma_{i} \frac{\left|\nabla \mu_{i h}^{n+1}\right|^{2}}{\Sigma_{i}^{2}} \geqslant \Sigma \sum_{i=1}^{3} \frac{\left|\nabla \mu_{i h}^{n+1}\right|^{2}}{\Sigma_{i}^{2}} \geqslant 0, \quad \text { since } \sum_{i=1}^{3} \frac{\nabla \mu_{i h}^{n+1}}{\Sigma_{i}}=0 \text { (Thm. 2.6), }
$$

and

$$
\sum_{i=1}^{3} \Sigma_{i}\left|\nabla c_{i h}^{n+1}-\nabla c_{i h}^{n}\right|^{2} \geqslant \underline{\Sigma} \sum_{i=1}^{3}\left|\nabla c_{i h}^{n+1}-\nabla c_{i h}^{n}\right|^{2} \geqslant 0, \quad \text { since } \sum_{i=1}^{3} \nabla\left(c_{i h}^{n+1}-c_{i h}^{n}\right)=0 \text { (Thm. 2.6). }
$$

Equality (2.16) is a discrete version of the energy equality satisfied by solutions $(\mathbf{c}, \boldsymbol{\mu})$ of the continuous Cahn-Hilliard system (1.3):

$$
\frac{\mathrm{d}}{\mathrm{d} t}\left[\mathcal{F}_{\mathbf{\Sigma}, \varepsilon}^{\text {triph }}(\mathbf{c})\right]=-\int_{\Omega} \sum_{i=1}^{3} \frac{M_{0}(\mathbf{c})}{\Sigma_{i}}\left|\nabla \mu_{i}\right|^{2} \mathrm{~d} x
$$

This equality shows in particular that the energy of solutions of system (1.3) decreases in time. At the discrete level, the energy equality (2.16) may provide not only the decrease of the discrete energy but also the a priori estimates required to prove existence of approximate solutions and their convergence towards a weak solution of (1.3). However, two additional terms appear in the discrete counterpart (2.16) and, consequently, the validity of the discrete free energy decrease and a priori estimates depend on the sign of these terms:

- The last term in the left-hand side of (2.16) is a standard numerical diffusion term due to the time discretization of " $\Delta c_{i}$ " in the second equation of (1.3). This term has a "good sign" when $\beta \geq 0.5$ (Rem. 2.8) and can be removed by setting $\beta=0.5$.

- The right-hand side of (2.16) involves the time discretization $\mathbf{d}^{F}$ of non linear terms and, consequently its sign depends on particular choices of $\mathbf{d}^{F}$. 
Thus, the discretization of nonlinear terms $\mathbf{d}^{F}$ may be chosen thanks to a study of the right-hand side of (2.16). The simplest situation is when $\mathbf{d}^{F}$ is such that this term is zero. In this case, the discrete energy equality exactly mimics the continuous one. When the right-hand side of (2.16) has a "good sign", i.e. is negative, it is still possible to eliminate it in order to obtain an energy inequality. More generally, it is sufficient to be able to control the right-hand side of (2.16) in order to obtain convenient a priori estimates (see Sect. 3.2). This is the reason why, in the following section, assumptions on the discretization of nonlinear terms are given under the form of estimates involving the terms of the energy equality (2.16). In both Theorems 2.9 and 2.10 , these assumptions are used to bound the approximate solution $\left(\mathbf{c}_{h}^{n+1}, \boldsymbol{\mu}_{h}^{n+1}\right.$ ) (in convenient norms). A key point is that, in existence theorem, bounds may depend on the solution at previous time $\mathbf{c}_{h}^{n}$, on the time step $\Delta t$ or on the mesh size $h$ (all these quantities are fixed here) whereas, in convergence theorem, this is crucial that these a priori estimates lead to bounds which are independent on the time step $\Delta t$ and the mesh size $h$. The different assumptions will be validated for all the schemes presented in Section 3.

\subsection{Existence and convergence theorems}

This subsection is devoted to state general existence and convergence theorems whose proofs are given in Section 4. First of all, we give general assumptions on the discretization of non linear terms $\mathbf{d}^{F}: \mathbb{R}^{3} \times \mathbb{R}^{3} \rightarrow \mathbb{R}^{3}$. The function $\mathbf{d}^{F}$ belongs to the $\mathrm{C}^{1}\left(\mathbb{R}^{3} \times \mathbb{R}^{3}\right)$ class and satisfies the following assumption of polynomial growth: there exist a constant $B_{1} \geqslant 0$ and a real $p$ such that $2 \leq p<+\infty$ if $d=2$ or $p=6$ if $d=3$ and

$$
\begin{array}{r}
\forall i \in\{1,2,3\}, \forall\left(\mathbf{a}^{n}, \mathbf{a}^{n+1}\right) \in \mathcal{S}^{2}, \quad\left|d_{i}^{F}\left(\mathbf{a}^{n}, \mathbf{a}^{n+1}\right)\right| \leqslant B_{1}\left(1+\left|\mathbf{a}^{n}\right|^{p-1}+\left|\mathbf{a}^{n+1}\right|^{p-1}\right), \\
\left|D\left(d_{i}^{F}\left(\mathbf{a}^{n}, \cdot\right)\right)\left(\mathbf{a}^{n+1}\right)\right| \leqslant B_{1}\left(1+\left|\mathbf{a}^{n}\right|^{p-2}+\left|\mathbf{a}^{n+1}\right|^{p-2}\right) .
\end{array}
$$

Theorem 2.9 (existence of a discrete solution). Let $\mathbf{c}_{h}^{n} \in \mathcal{V}_{D h, \mathcal{S}}^{\mathbf{c}}$ given. Assume that:

- the coefficients $\left(\Sigma_{1}, \Sigma_{2}, \Sigma_{3}\right)$ satisfy (1.5), the mobility satisfies (1.10), and the bulk energy $F$ satisfies (1.11);

- the discretization of the non linear terms $\mathbf{d}^{F}$ satisfies (2.20) and the following property: there exists $K_{1}^{\mathbf{c}_{h}^{n}}>0$ (possibly depending on $\mathbf{c}_{h}^{n}$ ) such that

$$
\int_{\Omega}\left[F\left(\mathbf{a}_{h}^{n+1}\right)-F\left(\mathbf{c}_{h}^{n}\right)-\mathbf{d}^{F}\left(\mathbf{c}_{h}^{n}, \mathbf{a}_{h}^{n+1}\right) \cdot\left(\mathbf{a}_{h}^{n+1}-\mathbf{c}_{h}^{n}\right)\right] \mathrm{d} x \leqslant K_{1}^{\mathbf{c}_{h}^{n}}, \quad \forall \mathbf{a}_{h}^{n+1} \in \mathcal{V}_{D h, \mathcal{S}}^{\mathbf{c}} .
$$

Then, there exists at least one solution $\left(\mathbf{c}_{h}^{n+1}, \boldsymbol{\mu}_{h}^{n+1}\right)$ of Problem 2.2.

For each $N \in \mathbb{N}$, we can now introduce piecewise defined functions of time, on $\left[0, t_{f}\right]$, defined as follows:

$$
\begin{gathered}
\left.\underline{c}_{i h}^{N}(t, \cdot)=c_{i h}^{n}(\cdot), \quad \text { if } t \in\right] t_{n}, t_{n+1}[, \\
\left.\bar{c}_{i h}^{N}(t, \cdot)=c_{i h}^{n+1}(\cdot), \quad \text { if } t \in\right] t_{n}, t_{n+1}[, \\
\left.c_{i h}^{N}(t, \cdot)=\frac{t_{n+1}-t}{\Delta t} c_{i h}^{n}(\cdot)+\frac{t-t_{n}}{\Delta t} c_{i h}^{n+1}(\cdot), \quad \text { if } t \in\right] t_{n}, t_{n+1}[.
\end{gathered}
$$

For the chemical potential, we introduce piecewise-constant functions in time: for each $N \in \mathbb{N}$, let

$$
\left.\mu_{i h}^{N}(t, \cdot)=\mu_{i h}^{n+1}(\cdot), \quad \text { if } t \in\right] t_{n}, t_{n+1}[\cdot
$$

Theorem 2.10 (convergence theorem). Assume that assumptions of Theorem 2.9 are satisfied so that the approximate solutions $\left(\mathbf{c}_{h}^{N}, \boldsymbol{\mu}_{h}^{N}\right)$ of Problem 2.2 exists for all $N \in \mathbb{N}^{*}$ and for all $h>0$. Assume that $\left.\left.\beta \in\right] \frac{1}{2}, 1\right]$, 
that the consistency property (2.3) holds and that there exists constants $C>0$ and $\Delta t_{0}>0$ such that for all $\Delta t \leqslant \Delta t_{0}$ and for all $n \in \llbracket 0, N-1 \rrbracket$,

$$
\begin{aligned}
\mathcal{F}_{\boldsymbol{\Sigma}, \varepsilon}^{\operatorname{triph}}\left(\mathbf{c}_{h}^{n+1}\right)-\mathcal{F}_{\boldsymbol{\Sigma}, \varepsilon}^{\operatorname{triph}}\left(\mathbf{c}_{h}^{n}\right) & \\
+C & {\left[\Delta t \sum_{i=1}^{3} \int_{\Omega} \frac{M_{0 h}^{n+\alpha}}{\Sigma_{i}}\left|\nabla \mu_{i h}^{n+1}\right|^{2} \mathrm{~d} x+\frac{3}{8}(2 \beta-1) \varepsilon \int_{\Omega} \sum_{i=1}^{3} \Sigma_{i}\left|\nabla c_{i h}^{n+1}-\nabla c_{i h}^{n}\right|^{2} \mathrm{~d} x\right] \leqslant 0 }
\end{aligned}
$$

Consider the problem (1.3) together with the initial condition (1.9) and boundary conditions (1.8). Then, there exists a weak solution $(\mathbf{c}, \boldsymbol{\mu})$ on $\left[0, t_{f}[\right.$ such that

$$
\begin{gathered}
\mathbf{c} \in \mathrm{L}^{\infty}\left(0, t_{f} ;\left(\mathrm{H}^{1}(\Omega)\right)^{3}\right) \cap \mathrm{C}^{0}\left(\left[0, t_{f}\left[;\left(\mathrm{L}^{q}(\Omega)\right)^{3}\right), \text { for all } q<6\right.\right. \\
\boldsymbol{\mu} \in \mathrm{L}^{2}\left(0, t_{f} ;\left(\mathrm{H}^{1}(\Omega)\right)^{3}\right), \\
\mathbf{c}(t, x) \in \mathcal{S}, \text { for a.e. }(t, x) \in\left[0, t_{f}[\times \Omega .\right.
\end{gathered}
$$

and for all sequences $\left(h_{K}\right)_{K \in \mathbb{N}^{*}}$ such that $h_{K} \underset{K \rightarrow+\infty}{\longrightarrow} 0$, the sequences $\left(\mathbf{c}_{h_{K}}^{N}\right)_{(N, K) \in\left(\mathbb{N}^{*}\right)^{2}}$ and $\left(\boldsymbol{\mu}_{h_{K}}^{N}\right)_{(N, K) \in\left(\mathbb{N}^{*}\right)^{2}}$, defined by (2.8), satisfy, up to a subsequence, the following convergences, when $\min (N, K) \longrightarrow+\infty$ :

$$
\begin{aligned}
& \mathbf{c}_{h_{K}}^{N} \rightarrow \mathbf{c} \quad \text { in } \mathrm{C}^{0}\left(0, t_{f},\left(\mathrm{~L}^{q}\right)^{3}\right) \text { strong, } \quad \text { for all } q<6 \\
& \boldsymbol{\mu}_{h_{K}}^{N} \rightarrow \boldsymbol{\mu} \quad \text { in } \mathrm{L}^{2}\left(0, t_{f},\left(\mathrm{H}^{1}\right)^{3}\right) \text { weak. }
\end{aligned}
$$

Remark 2.11. In Theorem 2.10, we assume that $\frac{1}{2}<\beta \leqslant 1$. Indeed, the last term in the left hand side of inequality (2.26) (which vanish in the case where $\beta$ is equal $\frac{1}{2}$ ) is crucial in the estimates of remainders (see Sect. 4.2.2) and in the proof of the energy estimate for the implicit scheme (see Sect. 3.2.2).

Remark 2.12. Under an additional assumption on the Hessian of the Cahn-Hilliard potential $F$, it is shown in [8] that the model (1.3) has a unique weak solution. In this case, we can conclude that the convergence in Theorem 2.10 holds for the entire sequences $\left(\mathbf{c}_{h_{K}}^{N}, \boldsymbol{\mu}_{h_{K}}^{N}\right)$.

\section{Different Discretizations FOR NON LinEAR TERMS}

In this section, we present different possible choices of the discretization of nonlinear terms $\mathbf{d}^{F}$. Since definition (1.12) provides a natural splitting of $F: F=F_{0}+P$, we will choose a discretization of the form $d_{i}^{F}=d_{i}^{F_{0}}+d_{i}^{P}$ where $d_{i}^{F_{0}}$ and $d_{i}^{P}$ represent a discretization of $\partial_{c_{i}} F_{0}$ and $\partial_{c_{i}} P$ respectively. We give three possible choices of the discretization of the contribution of $F_{0}$ in Sections 3.2, 3.3 and 3.4, and a semi-implicit discretization of the contribution of $P$ in Section 3.5. In each of these subsection, estimates on the corresponding contribution in the right-hand side of (2.16) are proven. The results are then gathered in Section 3.6 in order to get the existence of approximate solutions and their convergence towards a weak solution of (1.3). Finally in Section 3.7, we show that the algebraic consistency property (see Sect. 1.2) has its discrete counterpart by identifying schemes that we obtained when only two phases are present.

\subsection{Preliminary remark}

The relationship $c_{1}+c_{2}+c_{3}=1$ allows to find a useful equivalent expression of $F_{0}$ on the hyperplane $\mathcal{S}$. This expression involves the diphasic Cahn-Hilliard potential $f$ defined by:

$$
f(x)=x^{2}(1-x)^{2}, \quad \forall x \in \mathbb{R} .
$$


Indeed, the function defined by:

$$
\hat{F}_{0}(\mathbf{c})=\sum_{i=1}^{3} \frac{\Sigma_{i}}{2} f\left(c_{i}\right), \quad \forall \mathbf{c} \in \mathbb{R}^{3}
$$

is equal to $F_{0}$ on the hyperplane $\mathcal{S}$ :

$$
\hat{F}_{0}(\mathbf{c})=F_{0}(\mathbf{c}), \forall \mathbf{c} \in \mathcal{S}
$$

These two different expressions can be equivalently used since we can readily prove that

$$
\nabla F_{0}(\mathbf{c}) \cdot \boldsymbol{\xi}=\nabla \hat{F}_{0}(\mathbf{c}) \cdot \boldsymbol{\xi}, \quad \forall(\mathbf{c}, \boldsymbol{\xi}) \in \mathcal{S}^{2}
$$

and consequently,

$$
f_{i}^{\hat{F}_{0}}(\mathbf{c})=f_{i}^{F_{0}}(\mathbf{c}), \quad \forall \mathbf{c} \in \mathcal{S} .
$$

\subsection{Implicit discretization for the contribution of $\boldsymbol{F}_{\mathbf{0}}$}

The implicit discretization corresponds to the following definition:

$$
\mathbf{d}^{F_{0}}\left(\mathbf{a}^{n}, \mathbf{a}^{n+1}\right)=\nabla F_{0}\left(\mathbf{a}^{n+1}\right), \quad \forall\left(\mathbf{a}^{n}, \mathbf{a}^{n+1}\right) \in \mathcal{S}^{2} .
$$

In Sections 3.2.1 and 3.2.2, we respectively prove that the contribution of $F_{0}$ satisfies estimates (2.21) of Theorem 2.9 and (2.26) of Theorem 2.10 when using the implicit discretization (3.4). Note that we need to assume here that: $\Sigma_{i}>0, \forall i \in\{1,2,3\}$, that is the case of partial spreading situations.

In total spreading situations (i.e. when one of the $\Sigma_{i}$ is negative), the proof of existence and convergence theorems when using the implicit discretization (3.4) is still an open problem. In numerical experiments, we observe that, in this case, the Newton linearization method may fail to converge in the resolution of Problem 2.4 (see Tab. 4 in Sect. 5).

\subsubsection{Existence of discrete solution}

We prove here, in the case where all $\Sigma_{i}$ are positive, that assumption (2.21) of Theorem 2.9 holds for the contribution of $F_{0}$ when using the implicit discretization (3.4). The proof makes use of the expression (3.2) of $F_{0}$ (valid on the hyperplane $\mathcal{S}$ ) and of the preliminary remark given in Section 3.1.

Proposition 3.1. Let $\mathbf{c}_{h}^{n} \in \mathcal{V}_{D h, \mathcal{S}}^{\mathbf{c}}$. Assume that: $\forall i \in\{1,2,3\}, \Sigma_{i}>0$. Then, there exists $K_{1}^{\mathbf{c}_{h}^{n}}>0$ possibly depending on $\mathbf{c}_{h}^{n}$ such that:

$$
\int_{\Omega}\left[F_{0}\left(\mathbf{a}_{h}^{n+1}\right)-F_{0}\left(\mathbf{c}_{h}^{n}\right)-\nabla F_{0}\left(\mathbf{a}_{h}^{n+1}\right) \cdot\left(\mathbf{a}_{h}^{n+1}-\mathbf{c}_{h}^{n}\right)\right] \mathrm{d} x \leqslant K_{1}^{\mathbf{c}_{h}^{n}}, \quad \forall \mathbf{a}_{h}^{n+1} \in \mathcal{V}_{D h, \mathcal{S}}^{\mathbf{c}}
$$

Proof. We can readily see that there exists two constants $C_{1}$ and $C_{2}$ such that the function $f$ defined in (3.1) satisfies

$$
f(x)-f(y)-f^{\prime}(x)(x-y) \leqslant C_{1}+C_{2}|y|+|f(y)|, \quad \forall x \in \mathbb{R}, \forall y \in \mathbb{R} .
$$

Then, by combining (3.2) and (3.6), since all $\Sigma_{i}$ are positive, we have, for all $\mathbf{a}_{h}^{n+1} \in \mathcal{V}_{D h, \mathcal{S}}^{\mathbf{c}}$,

$$
\begin{aligned}
& \int_{\Omega} \hat{F}_{0}\left(\mathbf{a}_{h}^{n+1}\right)-\hat{F}_{0}\left(\mathbf{c}_{h}^{n}\right)- \nabla \hat{F}_{0}\left(\mathbf{a}_{h}^{n+1}\right) \cdot\left(\mathbf{a}_{h}^{n+1}-\mathbf{c}_{h}^{n}\right) \mathrm{d} x \\
& \leqslant C|\Omega| \sum_{i=1}^{3} \frac{\Sigma_{i}}{2}+C \sum_{i=1}^{3} \frac{\Sigma_{i}}{2} \int_{\Omega}\left|c_{i h}^{n}\right| \mathrm{d} x+C \sum_{i=1}^{3} \frac{\Sigma_{i}}{2} \int_{\Omega}\left|f\left(c_{i h}^{n}\right)\right| \mathrm{d} x:=K_{1}^{\mathbf{c}_{h}^{n}} .
\end{aligned}
$$

The conclusion is obtained thanks to equality (3.3). 


\subsubsection{Convergence of approximate solution}

The estimate of Proposition 3.1 holds for all time steps but is not sufficient to prove the convergence theorem. In this section we give here another estimate (which corresponds to assumption (2.26) of Thm. 2.10) only available for small enough time steps.

Proposition 3.2. Assume that: $\forall i \in\{1,2,3\}, \Sigma_{i}>0$ and that assumption (1.10) hold. Then, we get

$$
\mathcal{F}_{\boldsymbol{\Sigma}, \varepsilon}^{\mathrm{triph}}\left(\mathbf{c}_{h}^{n+1}\right)-\mathcal{F}_{\boldsymbol{\Sigma}, \varepsilon}^{\mathrm{triph}}\left(\mathbf{c}_{h}^{n}\right)+\frac{\Delta t}{2} \sum_{i=1}^{3} \int_{\Omega} \frac{M_{0 h}^{n+\alpha}}{\Sigma_{i}}\left|\nabla \mu_{i h}^{n+1}\right|^{2} \mathrm{~d} x+\frac{3}{16} \varepsilon(2 \beta-1) \int_{\Omega} \sum_{i=1}^{3} \Sigma_{i}\left|\nabla c_{i h}^{n+1}-\nabla c_{i h}^{n}\right|^{2} \mathrm{~d} x \leqslant 0
$$

as soon as $\Delta t \leqslant \Delta t_{0}=\frac{(2 \beta-1) \varepsilon^{3}}{24 M_{2}}$.

Proof. Considering the function $f$ defined by (3.1) and since $\inf _{\mathbb{R}} f^{\prime \prime}=-1$, we readily obtain

$$
f(x)-f(y)-f^{\prime}(x)(x-y) \leqslant \frac{(x-y)^{2}}{2}, \quad \forall x \in \mathbb{R}, \forall y \in \mathbb{R}
$$

Since all $\Sigma_{i}$ are positive, we get

$$
\begin{aligned}
\hat{F}_{0}\left(\mathbf{c}_{h}^{n+1}\right)-\hat{F}_{0}\left(\mathbf{c}_{h}^{n}\right)-\nabla \hat{F}_{0}\left(\mathbf{c}_{h}^{n+1}\right) \cdot\left(\mathbf{c}_{h}^{n+1}-\mathbf{c}_{h}^{n}\right) & =\sum_{i=1}^{3} \frac{\Sigma_{i}}{2}\left(f\left(c_{i h}^{n+1}\right)-f\left(c_{i h}^{n}\right)-f^{\prime}\left(c_{i h}^{n+1}\right)\left(c_{i h}^{n+1}-c_{i h}^{n}\right)\right) \\
& \leqslant \sum_{i=1}^{3} \frac{\sum_{i}}{4}\left|c_{i h}^{n+1}-c_{i h}^{n}\right|^{2} .
\end{aligned}
$$

Owing to equalities (2.16) and (3.3), we find the estimate

$$
\begin{aligned}
\mathcal{F}_{\boldsymbol{\Sigma}, \varepsilon}^{\text {triph }}\left(\mathbf{c}_{h}^{n+1}\right)-\mathcal{F}_{\mathbf{\Sigma}, \varepsilon}^{\text {triph }}\left(\mathbf{c}_{h}^{n}\right) & +\Delta t \sum_{i=1}^{3} \int_{\Omega} \frac{M_{0 h}^{n+\alpha}}{\Sigma_{i}}\left|\nabla \mu_{i h}^{n+1}\right|^{2} \mathrm{~d} x \\
& \leqslant \frac{3}{\varepsilon} \int_{\Omega} \sum_{i=1}^{3} \Sigma_{i}\left|c_{i h}^{n+1}-c_{i h}^{n}\right|^{2} \mathrm{~d} x-\frac{3}{8} \varepsilon(2 \beta-1) \int_{\Omega} \sum_{i=1}^{3} \Sigma_{i}\left|\nabla c_{i h}^{n+1}-\nabla c_{i h}^{n}\right|^{2} \mathrm{~d} x
\end{aligned}
$$

In order to bound the term $\sum_{i=1}^{3} \Sigma_{i}\left|c_{i h}^{n+1}-c_{i h}^{n}\right|^{2}$, we take $\nu_{h}^{\mu}=\Sigma_{i}\left(c_{i h}^{n+1}-c_{i h}^{n}\right)$ as a test function in the first equation of (2.8) (remark that $\nu_{h}^{\mu} \in \mathcal{V}_{h}^{\mu}$ since $\mathcal{V}_{D h, 0}^{c} \subset \mathcal{V}_{h}^{\mu}$ (assumption (2.7))). Hence, we obtain

$$
\int_{\Omega} \Sigma_{i} \frac{c_{i h}^{n+1}-c_{i h}^{n}}{\Delta t}\left(c_{i h}^{n+1}-c_{i h}^{n}\right) \mathrm{d} x=-\int_{\Omega} \Sigma_{i} \frac{M_{0 h}^{n+\alpha}}{\Sigma_{i}} \nabla \mu_{i h}^{n+1} \cdot \nabla\left(c_{i h}^{n+1}-c_{i h}^{n}\right) \mathrm{d} x .
$$

Adding these equations for $i=1,2,3$, and applying Corollary 1.2, yield

$$
\int_{\Omega} \sum_{i=1}^{3} \Sigma_{i}\left|c_{i h}^{n+1}-c_{i h}^{n}\right|^{2} \mathrm{~d} x \leqslant \frac{\Delta t}{2} \int_{\Omega} M_{0 h}^{n+\alpha}\left[\frac{\varepsilon}{3} \sum_{i=1}^{3} \frac{\left|\nabla \mu_{i h}^{n+1}\right|^{2}}{\Sigma_{i}}+\frac{3}{\varepsilon} \sum_{i=1}^{3} \Sigma_{i}\left|\nabla c_{i h}^{n+1}-\nabla c_{i h}^{n}\right|^{2}\right] \mathrm{d} x .
$$

Using (1.10), we get

$$
\frac{3}{\varepsilon} \int_{\Omega} \sum_{i=1}^{3} \Sigma_{i}\left|c_{i h}^{n+1}-c_{i h}^{n}\right|^{2} \mathrm{~d} x \leqslant \frac{\Delta t}{2} \sum_{i=1}^{3} \int_{\Omega} \frac{M_{0 h}^{n+\alpha}}{\Sigma_{i}}\left|\nabla \mu_{i h}^{n+1}\right|^{2} \mathrm{~d} x+\frac{9 M_{2} \Delta t}{2 \varepsilon^{2}} \sum_{i=1}^{3} \int_{\Omega} \Sigma_{i}\left|\nabla c_{i h}^{n+1}-\nabla c_{i h}^{n}\right|^{2} \mathrm{~d} x
$$


Thus, by combining the inequality (3.8) and (3.9), we finally have

$$
\begin{aligned}
\mathcal{F}_{\Sigma, \varepsilon}^{\text {triph }}\left(\mathbf{c}_{h}^{n+1}\right)-\mathcal{F}_{\Sigma, \varepsilon}^{\text {triph }}\left(\mathbf{c}_{h}^{n}\right)+\Delta t \sum_{i=1}^{3} \int_{\Omega} \frac{M_{0 h}^{n+\alpha}}{\Sigma_{i}}\left|\nabla \mu_{i h}^{n+1}\right|^{2} \mathrm{~d} x \leqslant & \frac{\Delta t}{2} \sum_{i=1}^{3} \int_{\Omega} \frac{M_{0 h}^{n+\alpha}}{\Sigma_{i}}\left|\nabla \mu_{i h}^{n+1}\right|^{2} \mathrm{~d} x \\
& +\frac{9 M_{2} \Delta t}{2 \varepsilon^{2}} \sum_{i=1}^{3} \int_{\Omega} \Sigma_{i}\left|\nabla c_{i h}^{n+1}-\nabla c_{i h}^{n}\right|^{2} \mathrm{~d} x \\
& -\frac{3}{8} \varepsilon(2 \beta-1) \int_{\Omega} \sum_{i=1}^{3} \Sigma_{i}\left|\nabla c_{i h}^{n+1}-\nabla c_{i h}^{n}\right|^{2} \mathrm{~d} x
\end{aligned}
$$

The conclusion is readily obtained by using that $\Delta t \leqslant \frac{(2 \beta-1) \varepsilon^{3}}{24 M_{2}}$.

\subsection{Convex-concave discretization for the contribution of $\boldsymbol{F}_{\mathbf{0}}$}

In Section 3.2, we saw that the implicit scheme (3.4) ensures the decrease of the discrete energy only for small enough time steps. Moreover, the results hold only in the case of partial spreading situations. To overcome these difficulties, in this subsection, we look for a discretization $\mathbf{d}^{F_{0}}$ such that:

$$
F_{0}\left(\mathbf{a}^{n+1}\right)-F_{0}\left(\mathbf{a}^{n}\right)-\mathbf{d}^{F_{0}}\left(\mathbf{a}^{n}, \mathbf{a}^{n+1}\right) \cdot\left(\mathbf{a}^{n+1}-\mathbf{a}^{n}\right) \leqslant 0, \quad \forall\left(\mathbf{a}^{n}, \mathbf{a}^{n+1}\right) \in \mathcal{S}^{2} .
$$

Assume for a moment that $\mathbf{d}^{F_{0}}$ is the implicit discretization (3.4); the inequality (3.10) would hold if the function $F_{0}$ was convex on the hyperplane $\mathcal{S}$. In the same manner, when using the explicit discretization, the inequality (3.10) would hold if the function $F_{0}$ was concave on the hyperplane $\mathcal{S}$. The potential $F_{0}$ is neither convex nor concave, nevertheless these remarks provides a natural way (see the pioneering works [18] for diphasic Cahn-Hilliard equation) to obtain a discretization $\mathbf{d}^{F_{0}}$ which satisfies (3.10) assuming that the function $F_{0}$ is decomposed as the sum of a convex function and a concave one. Indeed, if $F_{0}=F_{0}^{+}+F_{0}^{-}$with $F_{0}^{+}$convex and $F_{0}^{-}$concave then we can define

$$
\mathbf{d}^{F_{0}}\left(\mathbf{a}^{n}, \mathbf{a}^{n+1}\right)=\nabla F_{0}^{+}\left(\mathbf{a}^{n+1}\right)+\nabla F_{0}^{-}\left(\mathbf{a}^{n}\right) .
$$

In our case, the diphasic Cahn-Hilliard potential is naturally written with a convex-concave decomposition:

$$
f(x)=\underbrace{\left(x-\frac{1}{2}\right)^{4}}_{f^{+}(x)}+\underbrace{\frac{1}{16}\left(1-2(2 x-1)^{2}\right)}_{f^{-}(x)} .
$$

This decomposition readily leads to a convex-concave decomposition of $\hat{F}_{0}$ and to the following definitions:

$$
\begin{aligned}
& F_{0}^{+}(\mathbf{c})=\sum_{i=1}^{3} \frac{\Sigma_{i}^{+}}{2} f^{+}\left(c_{i}\right)-\sum_{i=1}^{3} \frac{\Sigma_{i}^{-}}{2} f^{-}\left(c_{i}\right) \\
& F_{0}^{-}(\mathbf{c})=\sum_{i=1}^{3} \frac{\Sigma_{i}^{+}}{2} f^{-}\left(c_{i}\right)-\sum_{i=1}^{3} \frac{\Sigma_{i}^{-}}{2} f^{+}\left(c_{i}\right),
\end{aligned}
$$

where $\Sigma_{i}^{+}=\max \left(\Sigma_{i}, 0\right)$ and $\Sigma_{i}^{-}=-\min \left(\Sigma_{i}, 0\right)$.

Since $F_{0}$ and $\hat{F}_{0}$ coincide on the hyperplane $\mathcal{S}$ (see (3.3)), the inequality (3.10) holds and thus assumptions (2.21) of Theorem 2.9 and (2.26) of Theorem 2.10 hold for the contribution of $F_{0}$ when using the convexconcave discretization (3.11) (see Sect. 3.6 for more details). These assumptions are satisfied for all time steps $\Delta t$ and even in the case of total spreading situations. 


\subsection{Semi-implicit discretization for the contribution of $\boldsymbol{F}_{\mathbf{0}}$}

The convex-concave scheme presented in Section 3.3 ensures the decrease of the energy for all time step and even in total spreading situations. However, it suffers from an important lack of accuracy (see Figs. 3 and 15 in Sect. 5). This is certainly due to the fact that the convex-concave discretization unequally splits the two parts of the Cahn-Hilliard potential which would act together or rather enter in competition. We propose here a more specific semi-implicit discretization built in order to obtain

$$
F_{0}\left(\mathbf{a}^{n+1}\right)-F_{0}\left(\mathbf{a}^{n}\right)-\mathbf{d}^{F_{0}}\left(\mathbf{a}^{n}, \mathbf{a}^{n+1}\right) \cdot\left(\mathbf{a}^{n+1}-\mathbf{a}^{n}\right)=0, \quad \forall\left(\mathbf{a}^{n}, \mathbf{a}^{n+1}\right) \in \mathcal{S}^{2}
$$

In $[24,25]$, the authors give other semi-implicit discretizations obtained thanks to Taylor expansion of the CahnHilliard potential.

In order to simplify notation, we denote $\mathbf{a}:=\mathbf{a}^{n}$ and $\mathbf{b}:=\mathbf{a}^{n+1}$ in the following calculation. We write $F_{0}(\mathbf{b})-F_{0}(\mathbf{a})$ as a sum of terms containing $\delta_{1}, \delta_{2}$ or $\delta_{3}$ in factor where $\delta_{i}=b_{i}-a_{i}$ for $i=1,2,3$. Since $F_{0}\left(c_{1}, c_{2}, c_{3}\right)=\sigma_{12} c_{1}^{2} c_{2}^{2}+\sigma_{13} c_{1}^{2} c_{3}^{2}+\sigma_{23} c_{2}^{2} c_{3}^{2}+c_{1} c_{2} c_{3}\left(\Sigma_{1} c_{1}+\Sigma_{2} c_{2}+\Sigma_{3} c_{3}\right)$, it is sufficient to separately consider terms of the form $b_{i}^{2} b_{j} b_{k}-a_{i}^{2} a_{j} a_{k}$ with $(i, j, k) \in\{1,2,3\}^{3}$. We use the identities $a_{i}^{2}=b_{i}^{2}-\left(a_{i}+b_{i}\right) \delta_{i}$ and $a_{j}=b_{j}-\delta_{j}$ in order to introduce $\delta_{i}, \delta_{j}$ and $\delta_{k}$ in the formula:

$$
\begin{aligned}
b_{i}^{2} b_{j} b_{k}-a_{i}^{2} a_{j} a_{k} & =b_{i}^{2}\left(b_{j} b_{k}-a_{j} a_{k}\right)+\left(a_{i}+b_{i}\right) a_{j} a_{k} \delta_{i} \\
& =b_{i}^{2}\left(b_{j} \delta_{k}+a_{k} \delta_{j}\right)+\left(a_{i}+b_{i}\right) a_{j} a_{k} \delta_{i} \\
& =\left(a_{i}+b_{i}\right) a_{j} a_{k} \delta_{i}+b_{i}^{2} a_{k} \delta_{j}+b_{i}^{2} b_{j} \delta_{k} .
\end{aligned}
$$

We now use this expression to build a symmetric formula in order to obtain, at least formally, a second order convergent discretization. By inverting the roles of $j$ and $k$, we can readily find

$$
b_{i}^{2} b_{j} b_{k}-a_{i}^{2} a_{j} a_{k}=\left(a_{i}+b_{i}\right) a_{j} a_{k} \delta_{i}+\frac{1}{2} b_{i}^{2}\left(a_{k}+b_{k}\right) \delta_{j}+\frac{1}{2} b_{i}^{2}\left(a_{j}+b_{j}\right) \delta_{k},
$$

and finally, by inverting the roles of $\mathbf{a}$ and $\mathbf{b}$, we get

$$
b_{i}^{2} b_{j} b_{k}-a_{i}^{2} a_{j} a_{k}=\frac{1}{2}\left(a_{i}+b_{i}\right)\left(a_{j} a_{k}+b_{j} b_{k}\right) \delta_{i}+\frac{1}{4}\left(a_{i}^{2}+b_{i}^{2}\right)\left(a_{k}+b_{k}\right) \delta_{j}+\frac{1}{4}\left(a_{i}^{2}+b_{i}^{2}\right)\left(a_{j}+b_{j}\right) \delta_{k} .
$$

We obtain a formula for terms of the form $b_{i}^{2} b_{j}^{2}-a_{i}^{2} a_{j}^{2}$ by taking $k=j$ in (3.14):

$$
b_{i}^{2} b_{j}^{2}-a_{i}^{2} a_{j}^{2}=\frac{1}{2}\left(a_{i}+b_{i}\right)\left(a_{j}^{2}+b_{j}^{2}\right) \delta_{i}+\frac{1}{2}\left(a_{i}^{2}+b_{i}^{2}\right)\left(a_{j}+b_{j}\right) \delta_{j}
$$

Hence, we propose to define, for any $i \in\{1,2,3\}$, the following consistent approximation of the non linear terms:

$$
\begin{aligned}
d_{i}^{F_{0}}\left(\mathbf{a}^{n}, \mathbf{a}^{n+1}\right)= & \frac{\Sigma_{i}}{4}\left[a_{i}^{n+1}+a_{i}^{n}\right]\left[\left(a_{j}^{n+1}+a_{k}^{n+1}\right)^{2}+\left(a_{j}^{n}+a_{k}^{n}\right)^{2}\right] \\
& +\frac{\Sigma_{j}}{4}\left[\left(a_{j}^{n+1}\right)^{2}+\left(a_{j}^{n}\right)^{2}\right]\left[a_{i}^{n+1}+a_{k}^{n+1}+a_{i}^{n}+a_{k}^{n}\right] \\
& +\frac{\Sigma_{k}}{4}\left[\left(a_{k}^{n+1}\right)^{2}+\left(a_{k}^{n}\right)^{2}\right]\left[a_{i}^{n+1}+a_{j}^{n+1}+a_{i}^{n}+a_{j}^{n}\right]
\end{aligned}
$$

we can readily deduce from the definition of $F_{0}$ and the formula (3.14) and (3.15) that, for any

$$
F_{0}\left(\mathbf{a}^{n+1}\right)-F_{0}\left(\mathbf{a}^{n}\right)=\sum_{i=1}^{3} d_{i}^{F_{0}}\left(\mathbf{a}^{n}, \mathbf{a}^{n+1}\right)\left(a_{i}^{n+1}-a_{i}^{n}\right), \quad \forall\left(\mathbf{a}^{n}, \mathbf{a}^{n+1}\right) \in \mathcal{S}^{2},
$$


and for any $\mathbf{c} \in \mathcal{S}$,

$$
d_{i}^{F_{0}}(\mathbf{c}, \mathbf{c})=\frac{\partial F_{0}}{\partial c_{i}}(\mathbf{c})
$$

Thus, from equality (3.13), we can deduce that assumptions (2.21) of Theorem 2.9 and (2.26) of Theorem 2.10 hold for the contribution of $F_{0}$ when using the semi-implicit discretization (3.16) (see Sect. 3.6 for more details). These assumptions are satisfied for all time steps $\Delta t$ and even in the case of total spreading situations.

\subsection{Semi-implicit discretization for the contribution of $P$}

Recall the definition of $P$ :

$$
P(\mathbf{c})=3 \Lambda c_{1}^{2} c_{2}^{2} c_{3}^{2}
$$

We only consider a semi-implicit discretization of the contribution of $P$ since numerical experiments from [26] shows the difficulties to use an implicit discretization for this term (non convergence of the Newton linearization method in the resolution of Problem 2.4). Moreover, we do not have a natural convex-concave decomposition of $P$. Hence, in order to obtain an energy estimate, we look for functions $d_{1}^{P}, d_{2}^{P}$ and $d_{3}^{P}$ such that $d_{i}^{P}(\mathbf{c}, \mathbf{c})=$ $\frac{\partial P}{\partial c_{i}}(\mathbf{c}), \forall \mathbf{c} \in \mathcal{S}$ and

$$
P\left(\mathbf{a}^{n+1}\right)-P\left(\mathbf{a}^{n}\right)-\mathbf{d}^{P}\left(\mathbf{a}^{n}, \mathbf{a}^{n+1}\right) \cdot\left(\mathbf{a}^{n+1}-\mathbf{a}^{n}\right)=0, \quad \forall\left(\mathbf{a}^{n}, \mathbf{a}^{n+1}\right) \in \mathcal{S}^{2} .
$$

As in the previous subsection, we define, for $i \in\{1,2,3\}, \delta_{i}=b_{i}-a_{i}$ and we use the identity $a_{i}^{2}=b_{i}^{2}-\left(a_{i}+b_{i}\right) \delta_{i}$ and then the equality (3.15) in order to introduce $\delta_{i}, \delta_{j}$ and $\delta_{k}$ in the term $b_{i}^{2} b_{j}^{2} b_{k}^{2},(i, j, k) \in\{1,2,3\}^{3}$ :

$$
\begin{aligned}
b_{i}^{2} b_{j}^{2} b_{k}^{2}-a_{i}^{2} a_{j}^{2} a_{k}^{2} & =b_{i}^{2}\left(b_{j}^{2} b_{k}^{2}-a_{j}^{2} a_{k}^{2}\right)+\left(a_{i}+b_{i}\right) a_{j}^{2} a_{k}^{2} \delta_{i} \\
& =\left(a_{i}+b_{i}\right) a_{j}^{2} a_{k}^{2} \delta_{i}+\frac{1}{2} b_{i}^{2}\left(a_{j}+b_{j}\right)\left(a_{k}^{2}+b_{k}^{2}\right) \delta_{j}+\frac{1}{2} b_{i}^{2}\left(a_{j}^{2}+b_{j}^{2}\right)\left(a_{k}+b_{k}\right) \delta_{k} .
\end{aligned}
$$

Adding the three formulas given by (3.18) with $(i, j, k)=(1,2,3),(2,1,3)$ and $(3,1,2)$ yields to

$$
\begin{aligned}
b_{1}^{2} b_{2}^{2} b_{3}^{2}-a_{1}^{2} a_{2}^{2} a_{3}^{2}= & \frac{1}{3}\left[a_{2}^{2} a_{3}^{2}+\frac{1}{2} b_{2}^{2} a_{3}^{2}+\frac{1}{2} a_{2}^{2} b_{3}^{2}+b_{2}^{2} b_{3}^{2}\right]\left(a_{1}+b_{1}\right) \delta_{1} \\
& +\frac{1}{3}\left[a_{1}^{2} a_{3}^{2}+\frac{1}{2} b_{1}^{2} a_{3}^{2}+\frac{1}{2} a_{1}^{2} b_{3}^{2}+b_{1}^{2} b_{3}^{2}\right]\left(a_{2}+b_{2}\right) \delta_{2} \\
& +\frac{1}{3}\left[a_{1}^{2} a_{2}^{2}+\frac{1}{2} b_{1}^{2} a_{2}^{2}+\frac{1}{2} a_{1}^{2} b_{2}^{2}+b_{1}^{2} b_{2}^{2}\right]\left(a_{3}+b_{3}\right) \delta_{3} .
\end{aligned}
$$

Thus, by defining

$$
d_{i}^{P}\left(\mathbf{a}^{n}, \mathbf{a}^{n+1}\right)=\Lambda\left(a_{i}^{n}+a_{i}^{n+1}\right)\left[\left(a_{j}^{n}\right)^{2}\left(a_{k}^{n}\right)^{2}+\frac{1}{2}\left(a_{j}^{n+1}\right)^{2}\left(a_{k}^{n}\right)^{2}+\frac{1}{2}\left(a_{j}^{n}\right)^{2}\left(a_{k}^{n+1}\right)^{2}+\left(a_{j}^{n+1}\right)^{2}\left(a_{k}^{n+1}\right)^{2}\right]
$$

we get property (3.17) and for any $\mathbf{c} \in \mathcal{S}$,

$$
d_{i}^{P}(\mathbf{c}, \mathbf{c})=\frac{\partial P}{\partial c_{i}}(\mathbf{c})
$$

Thus, as in previous subsection, from inequality (3.17), we can deduced that assumptions (2.21) of Theorem 2.9 and (2.26) of Theorem 2.10 hold for the contribution of $P$ when using the semi-implicit discretization (3.19) (see Sect. 3.6 for more details). 
TABLE 1. Summary of theoretical results.

\begin{tabular}{|c|c|c|c|c|c|}
\hline & \multicolumn{2}{|c|}{ Schemes } & Implicit & Convex-concave & Semi-implicit \\
\hline & \multirow{2}{*}{ Def. } & $\mathbf{d}^{P}$ & \multicolumn{3}{|c|}{ Semi-implicit (3.19) } \\
\hline & & $\mathbf{d}^{F_{0}}$ & Implicit (3.4) & Convex-concave (3.11) & Semi-implicit (3.16) \\
\hline$\forall i, \Sigma_{i}>0$ & \multicolumn{2}{|c|}{$\begin{array}{c}\mathbf{d}^{F}=\mathbf{d}^{F_{0}}+\mathbf{d}^{P} \\
\Lambda \geqslant 0\end{array}$} & $\begin{array}{c}\text { Decrease of energy } \Delta t \leqslant \Delta t_{0} \\
\text { Existence } \forall \Delta t \\
\text { Convergence }(\beta>1 / 2)\end{array}$ & \multicolumn{2}{|c|}{$\begin{array}{c}\text { Decrease of energy } \forall \Delta t \\
\text { Existence } \forall \Delta t \\
\text { Convergence }(\beta>1 / 2)\end{array}$} \\
\hline $\begin{array}{l}\quad \exists i, \Sigma_{i}<0 \\
\text { s.t. (1.5) holds }\end{array}$ & \multicolumn{2}{|c|}{$\begin{array}{c}\mathbf{d}^{F}=\mathbf{d}^{F_{0}}+\mathbf{d}^{P} \\
\Lambda \geqslant \Lambda_{0}\end{array}$} & Open problems & \multicolumn{2}{|c|}{$\begin{array}{c}\text { Decrease of energy } \forall \Delta t \\
\text { Existence } \forall \Delta t \\
\text { Convergence }(\beta>1 / 2)\end{array}$} \\
\hline
\end{tabular}

\subsection{Summary}

In previous Sections 3.2-3.5, we separately presented several discretizations $\mathbf{d}^{F_{0}}$ for the contribution of $F_{0}$ and a discretization $\mathbf{d}^{P}$ for the contribution of $P$ (recall that the Cahn-Hilliard potential $F$ is defined by $F=F_{0}+P$ ). For the contribution of $P$, we only consider the semi-implicit discretization (3.19) which is then combined with three possible discretizations for the contribution of $F_{0}$ : when we use the discretization (3.4), resp. (3.11), resp. (3.16), we refer to the scheme we obtained as the implicit, resp. convex-concave, resp. semi-implicit one.

We can now state existence and convergence theorems thanks to general Theorems 2.9 and 2.10 and to estimates (3.5), (3.7), (3.10) and (3.13) valid for particular discretizations. First of all, recall that the CahnHilliard potential $F=F_{0}+P$ satisfies assumption (1.11) (in fact only non negativity is not trivial, see Prop. 1.6) provided that:

- $\Lambda \geqslant 0$ when $\Sigma_{i}>0$ for all $i \in\{1,2,3\}$;

- $\Lambda \geqslant \Lambda_{0}$ when assumption (1.5) is satisfied (it allows the existence of at most one negative $\Sigma_{i}$ ).

In the first case, existence and convergence theorems are proven for the three schemes (implicit, convex-concave and semi-implicit one) whereas when one of the $\Sigma_{i}$ is negative, existence and convergence theorems are proven only for convex-concave and semi-implicit schemes, the existence of a solution for the implicit scheme being still an open problem. Note that in this last case, we observe, in some numerical experiments (see Sect. 5), a non convergence of the Newton linearization method in the resolution of Problem 2.4. In the case where all $\Sigma_{i}$ are positive, we can also remark that the implicit scheme ensures the decrease of the energy only for small enough time step (see Prop. 3.2) whereas convex-concave or semi-implicit scheme guarantees the decrease of energy for all time step. All these results are stated in Propositions 3.3 and 3.4 and summarized in Table 1.

Proposition 3.3 (partial spreading). Assume that: $\forall i \in\{1,2,3\}, \Sigma_{i}>0$, that $F=F_{0}+P$ with $\Lambda \geqslant 0$ and that the mobility satisfies (1.10) Then, there exists solutions of Problem 2.2 where $\mathbf{d}^{F}$ corresponds to the implicit, convex-concave or semi-implicit scheme. Moreover, these solutions satisfies the conclusions of Theorem 2.10 provided that $\frac{1}{2}<\beta \leqslant 1$.

Proposition 3.4 (total spreading). Assume that the triple of coefficients $\boldsymbol{\Sigma}$ satisfy (1.5), that $F=F_{0}+P$ with $\Lambda \geqslant \Lambda_{0}$ (see Prop. 1.6) and that the mobility satisfies (1.10). Then, there exists solutions of Problem 2.2 where $\mathbf{d}^{F}$ corresponds to the convex-concave or semi-implicit scheme. Moreover, these solutions satisfies the conclusions of Theorem 2.10 provided that $\frac{1}{2}<\beta \leqslant 1$.

\subsection{Corresponding schemes in the diphasic case}

Consider a system with two components (denoted below with underscripts 1 and 2 respectively) and assume that the evolution of the associated order parameters $c_{i}(i=1,2)$, and the associated chemical potentials $\tilde{\mu}_{i}$ 
$(i=1,2)$ of these two phases is governed by the diphasic Cahn-Hilliard model:

$$
\left\{\begin{aligned}
\frac{\partial c_{i}}{\partial t} & =\nabla \cdot\left(M\left(c_{1}, c_{2}\right) \nabla \tilde{\mu}_{i}\right), & & \text { for } i=1,2, \\
\tilde{\mu}_{i} & =\frac{12}{\varepsilon} \sigma_{12} f^{\prime}\left(c_{i}\right)-\frac{3}{2} \varepsilon \sigma_{12} \Delta c_{i}, & & \text { for } i=1,2,
\end{aligned}\right.
$$

where $\varepsilon$ stands for the interface thickness, $M\left(c_{1}, c_{2}\right)$ is a diffusion coefficient called mobility and $\sigma_{12}$ is the surface tension between the two components. The unknowns are linked by the following relationship $c_{1}+c_{2}=1$ and $\tilde{\mu_{1}}+\tilde{\mu_{2}}=0$.

The algebraic consistency (see Sect. 1.2) ensures that the triple $\left(c_{1}, c_{2}=1-c_{1}, c_{3}=0\right)$ is a particular solution of the triphasic Cahn-Hilliard model (1.3) (with $M_{0}(\mathbf{c})=2 \sigma_{12} M\left(c_{1}, c_{2}\right)$ ) for any choice of the surface tensions $\sigma_{13}$ and $\sigma_{23}$ involving the third component. In this case, the ternary chemical potentials are given by $\mu_{i}=\frac{\Sigma_{i}}{2 \sigma_{12}} \tilde{\mu}_{i}$ for $i=1,2$ and $\mu_{3}=0$.

The same kind of results can be obtained for the full discrete system and we can identify the following corresponding schemes for the diphasic model (3.20): Given $\left(c_{i h}^{n}, \mu_{i h}^{n}\right) \in \mathcal{V}_{D h}^{c_{i}} \times \mathcal{V}_{h}^{\mu}$,

- Implicit scheme in the diphasic case: for $i=1,2$, find $\left(c_{i h}^{n+1}, \mu_{i h}^{n+1}\right) \in \mathcal{V}_{D h}^{c_{i}} \times \mathcal{V}_{h}^{\mu}$ s.t.

$$
\left\{\begin{aligned}
\int_{\Omega} \frac{c_{i h}^{n+1}-c_{i h}^{n}}{\Delta t} \nu_{h}^{\mu} \mathrm{d} x & =-\int_{\Omega} M\left(c_{1 h}^{n+\alpha}, c_{2 h}^{n+\alpha}\right) \nabla \tilde{\mu}_{i h}^{n+1} \nabla \nu_{h}^{\mu} \mathrm{d} x, \quad \forall \nu_{h}^{\mu} \in \mathcal{V}_{h}^{\mu}, \\
\int_{\Omega} \tilde{\mu}_{i h}^{n+1} \nu_{h}^{c} \mathrm{~d} x & =\frac{12}{\varepsilon} \sigma_{12} \int_{\Omega} f^{\prime}\left(c_{i h}^{n+1}\right) \nu_{h}^{c} \mathrm{~d} x+\frac{3}{2} \varepsilon \sigma_{12} \int_{\Omega} \nabla c_{i h}^{n+\beta} \nabla \nu_{h}^{c} \mathrm{~d} x, \quad \forall \nu_{h}^{c} \in \mathcal{V}_{D h, 0}^{c} .
\end{aligned}\right.
$$

- Convex-concave scheme in the diphasic case: for $i=1,2$, find $\left(c_{i h}^{n+1}, \mu_{i h}^{n+1}\right) \in \mathcal{V}_{D h}^{c_{i}} \times \mathcal{V}_{h}^{\mu}$ s.t.

$$
\left\{\begin{aligned}
\int_{\Omega} \frac{c_{i h}^{n+1}-c_{i h}^{n}}{\Delta t} \nu_{h}^{\mu} \mathrm{d} x= & -\int_{\Omega} M\left(c_{1 h}^{n+\alpha}, c_{2 h}^{n+\alpha}\right) \nabla \tilde{\mu}_{i h}^{n+1} \nabla \nu_{h}^{\mu} \mathrm{d} x, \quad \forall \nu_{h}^{\mu} \in \mathcal{V}_{h}^{\mu} \\
\int_{\Omega} \tilde{\mu}_{i h}^{n+1} \nu_{h}^{c} \mathrm{~d} x= & \frac{12}{\varepsilon} \sigma_{12} \int_{\Omega}\left[\left(f^{+}\right)^{\prime}\left(c_{i h}^{n+1}\right)+\left(f^{-}\right)^{\prime}\left(c_{i h}^{n}\right)\right] \nu_{h}^{c} \mathrm{~d} x \\
& +\frac{3}{2} \varepsilon \sigma_{12} \int_{\Omega} \nabla c_{i h}^{n+\beta} \nabla \nu_{h}^{c} \mathrm{~d} x, \quad \forall \nu_{h}^{c} \in \mathcal{V}_{D h, 0}^{c}
\end{aligned}\right.
$$

where $f=f^{+}+f^{-}$is the convex-concave decomposition of $f$ given in (3.12).

- Semi-implicit scheme in the diphasic case: for $i=1,2$, find $\left(c_{i h}^{n+1}, \mu_{i h}^{n+1}\right) \in \mathcal{V}_{D h}^{c_{i}} \times \mathcal{V}_{h}^{\mu}$ s.t.

$$
\left\{\begin{aligned}
\int_{\Omega} \frac{c_{i h}^{n+1}-c_{i h}^{n}}{\Delta t} \nu_{h}^{\mu} \mathrm{d} x= & -\int_{\Omega} M\left(c_{1 h}^{n+\alpha}, c_{2 h}^{n+\alpha}\right) \nabla \tilde{\mu}_{i h}^{n+1} \nabla \nu_{h}^{\mu} \mathrm{d} x, \quad \forall \nu_{h}^{\mu} \in \mathcal{V}_{h}^{\mu}, \\
\int_{\Omega} \tilde{\mu}_{i h}^{n+1} \nu_{h}^{c} \mathrm{~d} x= & \frac{12}{\varepsilon} \sigma_{12} \int_{\Omega}\left[f^{\prime}\left(\frac{c_{i h}^{n}+c_{i h}^{n+1}}{2}\right)-\frac{1}{2}\left(1-c_{i h}^{n}-c_{i h}^{n+1}\right)\left(c_{i h}^{n+1}-c_{i h}^{n}\right)^{2}\right] \nu_{h}^{c} \mathrm{~d} x \\
& +\frac{3}{2} \varepsilon \sigma_{12} \int_{\Omega} \nabla c_{i}^{n+\beta} \nabla \nu_{h}^{c} \mathrm{~d} x, \quad \forall \nu_{h}^{c} \in \mathcal{V}_{D h, 0}^{c} .
\end{aligned}\right.
$$

Proposition 3.5. The above diphasic schemes (3.21)-(3.23) have at least one solution. Moreover, defining $M_{0}=2 \sigma_{12} M, \mu_{i h}^{n+1}=\frac{\Sigma_{i}}{2 \sigma_{12}} \tilde{\mu}_{i h}^{n+1}$ for $i=1,2$ and $\mu_{3 h}^{n+1}=0$, we have that if $\left(\left(c_{1 h}^{n+1}, \tilde{\mu}_{1 h}^{n+1}\right),\left(c_{2 h}^{n+1}, \tilde{\mu}_{2 h}^{n+1}\right)\right)$ is a solution of (3.21), (3.22) or (3.23) respectively then $\left(\left(c_{1 h}^{n+1}, \mu_{1 h}^{n+1}\right),\left(c_{2 h}^{n+1}, \mu_{2 h}^{n+1}\right),(0,0)\right)$ is a solution of the corresponding three phase discrete problem (2.2) where $\mathbf{d}^{F}$ is given by (3.4), (3.11) or (3.16) respectively. 
Remark 3.6. The expression of the ternary chemical potential $\mu_{i}$ differs from the two phase chemical potential $\tilde{\mu}_{i}$ but the quantities of interest in our application are the order parameters which give the position of phases and the capillary forces $f_{\text {ca }}$ which are typically used for the coupling with Navier-Stokes equation in a complete diffuse-interface ternary flows modelling (see [10]). In the triphasic model, we use the expression $f_{\text {ca }}=\sum_{i=1}^{3} \mu_{i} \nabla c_{i}$. The key point is that in the case where $c_{3}=0$, we have

$$
\begin{aligned}
f_{\mathrm{ca}} & =\mu_{1} \nabla c_{1}+\mu_{2} \nabla c_{2} \\
& =\frac{\Sigma_{1}}{2 \sigma_{12}} \tilde{\mu_{1}} \nabla c_{1}+\frac{\Sigma_{2}}{2 \sigma_{12}}\left(-\tilde{\mu_{1}}\right) \nabla\left(1-c_{1}\right) \\
& =\tilde{\mu_{1} \nabla c_{1}},
\end{aligned}
$$

which is the classical expression of capillary forces in the diphasic case.

Remark 3.7. In [24], the authors propose a semi-implicit scheme for the discretization of the two phase CahnHilliard model based on a Taylor expansion. It is interesting to note that, when only two phases are present, the semi-implicit scheme presented in the present work is very close but not identical to the scheme given in [24]. Indeed, we have the following relationship:

$$
\begin{aligned}
{\left[f^{\prime}\left(\frac{c_{h}^{n}+c_{h}^{n+1}}{2}\right)-\frac{1}{2}(1-\right.} & \left.\left.c_{h}^{n}-c_{h}^{n+1}\right)\left(c_{h}^{n+1}-c_{h}^{n}\right)^{2}\right] \\
- & {\left[f^{\prime}\left(c_{h}^{n+1}\right)-\frac{f^{\prime \prime}\left(c_{h}^{n+1}\right)}{2}\left(c_{h}^{n+1}-c_{h}^{n}\right)+\frac{f^{\prime \prime \prime}\left(c_{h}^{n+1}\right)}{6}\left(c_{h}^{n+1}-c_{h}^{n}\right)^{2}\right]=\left(c_{h}^{n+1}-c_{h}^{n}\right)^{3}, }
\end{aligned}
$$

where the first term of the left hand side is the discretization of $f^{\prime}(c)$ proposed here and the second one is the discretization proposed in [24].

\section{Proofs of existence And CONVERGEnce of Approximate solutions}

Let us recall the following Poincaré like result which will be very useful in the sequel. We denote by $m(\theta)$ the mean value of any function $\theta \in \mathrm{L}^{1}(\Omega): m(\theta)=\frac{1}{|\Omega|} \int_{\Omega} \theta \mathrm{d} x$.

Lemma 4.1 (Poincaré inequality). Let $\theta$ be a given function in $\mathrm{H}^{1}(\Omega)$ such that $m(\theta) \neq 0$. There exists a constant $C_{p, \theta}>0$ such that

$$
\forall \nu \in \mathrm{H}^{1}(\Omega),|\nu|_{\mathrm{H}^{1}(\Omega)} \leqslant C_{p, \theta}\left[|\nabla \nu|_{\mathrm{L}^{2}(\Omega)}+|m(\nu \theta)|\right] .
$$

\subsection{Proof of Theorem 2.9}

We are going to prove the existence of the solution of problem (2.8). The key points are the a priori estimates given by the discrete energy estimate and the topological degree theory [11].

We introduce a parameter $\delta \in[0,1]$ and the following problem: Find $\left(c_{1 h}^{n+1}, c_{2 h}^{n+1}, \mu_{1 h}^{n+1}, \mu_{2 h}^{n+1}\right) \in \mathcal{V}_{D h}^{c_{1}} \times \mathcal{V}_{D h}^{c_{2}} \times$ $\left(\mathcal{V}_{h}^{\mu}\right)^{2}$ such that $\forall \nu_{h}^{c} \in \mathcal{V}_{D h, 0}^{c}, \forall \nu_{h}^{\mu} \in \mathcal{V}_{h}^{\mu}$, we have, for $i=1$ and 2 ,

$$
\left\{\begin{array}{l}
\int_{\Omega} \frac{c_{i h}^{n+1}-c_{i h}^{n}}{\Delta t} \nu_{h}^{\mu} \mathrm{d} x=-\int_{\Omega} \frac{M_{0 h \delta}^{n+\alpha}}{\Sigma_{i}} \nabla \mu_{i h}^{n+1} \cdot \nabla \nu_{h}^{\mu} \mathrm{d} x, \\
\int_{\Omega} \mu_{i h}^{n+1} \nu_{h}^{c} \mathrm{~d} x=\int_{\Omega} \delta D_{i}^{F}\left(\mathbf{c}_{h}^{n}, \mathbf{c}_{h}^{n+1}\right) \nu_{h}^{c} \mathrm{~d} x+\int_{\Omega} \frac{3}{4} \Sigma_{i} \varepsilon \nabla c_{i h}^{n+\beta} \cdot \nabla \nu_{h}^{c} \mathrm{~d} x,
\end{array}\right.
$$

with $M_{0 h \delta}^{n+\alpha}=M_{0}\left((1-\delta \alpha) \mathbf{c}_{h}^{n}+\delta \alpha \mathbf{c}_{h}^{n+1}\right)$. 
The equations (4.2) continuously depend on the parameter $\delta$ and reduces to the initial problem (2.10) when $\delta=1$. Since the problem is finite dimensional (the spatial approximation spaces are fixed here), it is then enough to show that the problem is well-posed for $\delta=0$ and that we have an a priori estimate for the solutions of (4.2) which is uniform with respect to $\delta$.

- Let us first obtain the a priori estimate. Problem (4.2) is very similar to (2.10) with $\delta F$ instead of $F$, $\delta \mathbf{d}^{F}\left(\mathbf{c}^{n}, \mathbf{c}^{n+1}\right)$ as a choice of the discretization of non linear terms and a slightly modified mobility. It is thus possible to apply Theorem 2.7(the modification of the mobility $M_{0 h}$ do not change the calculation) and to obtain the following equality:

$$
\begin{aligned}
& \mathcal{F}_{\mathbf{\Sigma}, \varepsilon, \delta}^{\text {triph }}\left(\mathbf{c}_{h}^{n+1}\right)-\mathcal{F}_{\mathbf{\Sigma}, \varepsilon, \delta}^{\text {triph }}\left(\mathbf{c}_{h}^{n}\right)+\Delta t \sum_{i=1}^{3} \int_{\Omega} \frac{M_{0 h \delta}^{n+\alpha}}{\Sigma_{i}}\left|\nabla \mu_{i h}^{n+1}\right|^{2} \mathrm{~d} x \\
& \quad+\frac{3}{8}(2 \beta-1) \varepsilon \int_{\Omega} \sum_{i=1}^{3} \Sigma_{i}\left|\nabla c_{i h}^{n+1}-\nabla c_{i h}^{n}\right|^{2} \mathrm{~d} x=\frac{12}{\varepsilon} \delta \int_{\Omega}\left[F\left(\mathbf{c}_{h}^{n+1}\right)-F\left(\mathbf{c}_{h}^{n}\right)-\mathbf{d}^{F}\left(\mathbf{c}_{h}^{n}, \mathbf{c}_{h}^{n+1}\right) \cdot\left(\mathbf{c}_{h}^{n+1}-\mathbf{c}_{h}^{n}\right)\right] \mathrm{d} x
\end{aligned}
$$

with $\mathcal{F}_{\boldsymbol{\Sigma}, \varepsilon, \delta}^{\text {triph }}\left(\mathbf{c}_{h}^{k}\right)=\int_{\Omega} \delta \frac{12}{\varepsilon} F\left(\mathbf{c}_{h}^{k}\right)+\sum_{i=1}^{3} \frac{3}{8} \varepsilon \Sigma_{i}\left|\nabla c_{i h}^{k}\right|^{2} \mathrm{~d} x$. By using the assumption (2.21) and Remark 2.8, we get

$$
\mathcal{F}_{\Sigma, \varepsilon, \delta}^{\text {triph }}\left(\mathbf{c}_{h}^{n+1}\right)+\Delta t \int_{\Omega} M_{0 h \delta}^{n+\alpha} \sum_{i=1}^{3} \frac{\left|\nabla \mu_{i h}^{n+1}\right|^{2}}{\Sigma_{i}} \mathrm{~d} x \leqslant \mathcal{F}_{\mathbf{\Sigma}, \varepsilon, \delta}^{\operatorname{triph}}\left(\mathbf{c}_{h}^{n}\right)+\delta \frac{12}{\varepsilon} K_{1}^{\mathbf{c}_{h}^{n}} .
$$

Since the mobility is bounded from below (assumption (1.10)) and thanks to Remark 2.8, the second term of the left-hand side of (4.3) is bounded from below:

$$
\int_{\Omega} M_{1} \underline{\Sigma} \sum_{i=1}^{3} \frac{\left|\nabla \mu_{i h}^{n+1}\right|^{2}}{\Sigma_{i}^{2}} \mathrm{~d} x \leqslant \int_{\Omega} M_{0 h \delta}^{n+\alpha} \sum_{i=1}^{3} \frac{\left|\nabla \mu_{i h}^{n+1}\right|^{2}}{\Sigma_{i}} \mathrm{~d} x
$$

Furthermore, since $F \geqslant 0$ and $\delta \leqslant 1$, we have

$$
\mathcal{F}_{\boldsymbol{\Sigma}, \varepsilon, \delta}^{\mathrm{triph}}\left(\mathbf{c}_{h}^{k}\right) \leqslant \mathcal{F}_{\boldsymbol{\Sigma}, \varepsilon}^{\mathrm{triph}}\left(\mathbf{c}_{h}^{k}\right)
$$

and then, owing to $(4.3),(4.4),(4.5)$ and Proposition 1.1, there exists a constant $K_{2}^{\mathbf{c}_{h}^{n}}=\mathcal{F}_{\boldsymbol{\Sigma}, \varepsilon}^{\text {triph }}\left(\mathbf{c}_{h}^{n}\right)+$ $\frac{12}{\varepsilon} K_{1}^{\mathbf{c}_{h}^{n}}>0$ independent of $\delta$ and $\mathbf{c}_{h}^{n+1}$ such that

$$
\int_{\Omega} \delta \frac{12}{\varepsilon} F\left(\mathbf{c}_{h}^{n+1}\right)+\frac{3}{8} \varepsilon \underline{\Sigma} \sum_{i=1}^{3}\left|\nabla c_{i h}^{n+1}\right|^{2} \mathrm{~d} x+\Delta t \int_{\Omega} M_{1} \underline{\Sigma} \sum_{i=1}^{3} \frac{\left|\nabla \mu_{i h}^{n+1}\right|^{2}}{\Sigma_{i}^{2}} \mathrm{~d} x \leqslant K_{2}^{\mathbf{c}_{h}^{n}} .
$$

Since $F$ is positive and $\delta \geqslant 0$, we obtained the following bound for the second and third terms of the left-hand side of (4.6): for $i=1,2,3$,

$$
\left|\nabla c_{i h}^{n+1}\right|_{L^{2}} \leq \sqrt{\frac{8}{3} \frac{K_{2}^{\mathbf{c}_{h}^{n}}}{\varepsilon \underline{\Sigma}}}:=K_{3}^{\mathbf{c}_{h}^{n}} \quad \text { and } \quad\left|\nabla \mu_{i h}^{n+1}\right|_{\mathrm{L}^{2}} \leq \max _{i=1,2,3}\left(\left|\Sigma_{i}\right|\right) \sqrt{\frac{K_{2}^{\mathbf{c}_{h}^{n}}}{M_{1} \underline{\Sigma} \Delta t}}:=K_{4}^{\mathbf{c}_{h}^{n}}
$$


We now use the discrete form of the volume conservation (2.9): $m\left(c_{i h}^{n+1}\right)=m\left(c_{i h}^{n}\right)$. Thus, thanks to the Poincaré inequality (4.1) (with $\theta \equiv 1$ ), there exists a positive constant $\mathrm{C}_{\mathrm{p}}$ such that

$$
\left|c_{i h}^{n+1}\right|_{\mathrm{H}^{1}(\Omega)} \leqslant \mathrm{C}_{\mathrm{p}}\left(\left|\nabla c_{i h}^{n+1}\right|_{\mathrm{L}^{2}}+m\left(c_{i h}^{n+1}\right)\right)=\mathrm{C}_{\mathrm{p}}\left(\left|\nabla c_{i h}^{n+1}\right|_{\mathrm{L}^{2}}+m\left(c_{i h}^{n}\right)\right),
$$

and then there exists a positive constant $K_{5}^{\mathbf{c}_{h}^{n}}=\mathrm{C}_{\mathrm{p}}\left(K_{3}^{\mathbf{c}_{h}^{n}}+m\left(c_{i h}^{n}\right)\right)$ independent of $\delta$ and $\mathbf{c}_{h}^{n+1}$ such that

$$
\left|c_{i h}^{n+1}\right|_{\mathrm{H}^{1}(\Omega)} \leq K_{5}^{\mathbf{c}_{h}^{n}} .
$$

It remains to bound the average value $m\left(\mu_{i h}^{n+1}\right)$. Because of Dirichlet boundary conditions on $\mathbf{c}$, constants do not belong to $\mathcal{V}_{D h, 0}^{c}$. Hence, we take a fixed function $\theta_{h}$ of $\mathcal{V}_{D h, 0}^{c}$ such that $m\left(\theta_{h}\right) \neq 0$. Since $\mathcal{R}_{\delta}^{c_{i}}=0$, we have

$$
m\left(\mu_{i h}^{n+1} \theta_{h}\right)=\int_{\Omega} \delta D_{i}^{F}\left(\mathbf{c}_{h}^{n+1}, \mathbf{c}_{h}^{n}\right) \theta_{h} \mathrm{~d} x+\int_{\Omega} \frac{3}{4} \Sigma_{i} \varepsilon \nabla c_{i h}^{n+\beta} \cdot \nabla \theta_{h} \mathrm{~d} x .
$$

This can be controlled by $\left|\mathbf{c}_{h}^{n+1}\right|_{\mathrm{H}^{1}(\Omega)}$ and $\left|\mathbf{c}_{h^{n}}^{n}\right|_{\mathrm{H}^{1}(\Omega)}$ under the assumption (2.20). Indeed, the polynomial growth (2.20) of $d_{i}^{F}$ implies that there exists a positive constant $C_{1}=\frac{16 \Sigma_{T}}{3 \Sigma_{m}} B_{1}$ such that

$$
\left|D_{i}^{F}\left(\mathbf{c}_{h}^{n+1}, \mathbf{c}_{h}^{n}\right)\right| \leqslant C_{1}\left(1+\left|\mathbf{c}_{h}^{n+1}\right|^{p-1}+\left|\mathbf{c}_{h}^{n}\right|^{p-1}\right) .
$$

Thus, since $\delta \leqslant 1$, and by using (4.7),

$$
\begin{aligned}
m\left(\mu_{i h}^{n+1} \theta_{h}\right) & \leqslant C_{1}\left|\theta_{h}\right|_{L^{\infty}(\Omega)}\left(|\Omega|+\left|\mathbf{c}_{h}^{n+1}\right|_{L^{p}-1}^{p-1}+\left|\mathbf{c}_{h^{n}}^{n}\right|_{\mathrm{L}^{p}-1}^{p-1}\right)+\frac{3}{4} \Sigma_{M} \varepsilon\left(\left|\nabla c_{i h}^{n}\right|_{\mathrm{L}^{2}}+\left|\nabla c_{i h}^{n+1}\right|_{\mathrm{L}^{2}}\right)\left|\nabla \theta_{h}\right|_{\mathrm{L}^{2}} \\
& \leqslant C_{1}\left|\theta_{h}\right|_{\mathrm{L}^{\infty}(\Omega)}\left(|\Omega|+\left(K_{5}^{\mathbf{c}_{h}^{n}}\right)^{p-1}+\left|\mathbf{c}_{h}^{n}\right|_{\mathrm{H}^{1}}^{p-1}\right)+\frac{3}{4} \Sigma_{M} \varepsilon\left(\left|c_{i h}^{n}\right|_{\mathrm{H}^{1}}+K_{5}^{\mathbf{c}_{h}^{n}}\right)\left|\theta_{h}\right|_{\mathrm{H}^{1}}:=K_{6}^{h, \mathbf{c}_{h}^{n}}
\end{aligned}
$$

Thanks to the Poincaré inequality (4.1), there exists a constant $\mathrm{C}_{\mathrm{p}, \theta_{h}}$ such that

$$
\left|\mu_{i h}^{n+1}\right|_{\mathrm{H}^{1}(\Omega)} \leqslant \mathrm{C}_{\mathrm{p}, \theta_{h}}\left(\left|\nabla \mu_{i h}^{n+1}\right|_{\mathrm{L}^{2}}+m\left(\mu_{i h}^{n+1} \theta_{h}\right)\right) \leqslant \mathrm{C}_{\mathrm{p}, \theta_{h}}\left(K_{4}^{\mathbf{c}_{h}^{n}}+K_{6}^{h, \mathbf{c}_{h}^{n}}\right) .
$$

Finally, collecting (4.7) and (4.8), we get an a priori estimate which is uniform with respect to $\delta \in[0,1]$. - Let us now show that problem (4.2) is well-posed for $\delta=0$. In that case, the problem is linear, and the equations for $i=1,2,3$ are decoupled. Thus it is enough to show that, for $i \in\{1, \ldots, 3\}$, $\left(\tilde{c}_{i h}^{n+1}, \mu_{i h}^{n+1}\right)=(0,0)$ is the unique solution to the homogeneous problem

$$
\int_{\Omega}\left[\tilde{c}_{i h}^{n+1} \nu_{h}^{\mu}+\frac{M_{0 h}^{n}}{\Sigma_{i}} \Delta t \nabla \mu_{i h}^{n+1} \cdot \nabla \nu_{h}^{\mu}\right] \mathrm{d} x+\int_{\Omega}\left[\frac{3}{4} \Sigma_{i} \varepsilon \beta \nabla \tilde{c}_{i h}^{n+1} \cdot \nabla \nu_{h}^{c}-\mu_{i h}^{n+1} \nu_{h}^{c}\right] \mathrm{d} x, \quad \forall \nu_{h}^{\mu} \in \mathcal{V}_{h}^{\mu}, \forall \nu_{h}^{c} \in \mathcal{V}_{D h, 0}^{c} .
$$

To this end, we take $\left(\nu_{h}^{c}, \nu_{h}^{\mu}\right)=\left(\tilde{c}_{i h}^{n+1}, \mu_{i h}^{n+1}\right)$ in $(4.9)$ and get:

$$
\int_{\Omega} \tilde{c}_{i h}^{n+1} \mu_{i h}^{n+1} \mathrm{~d} x+\int_{\Omega} \frac{M_{0 h}^{n}}{\Sigma_{i}} \Delta t\left|\nabla \mu_{i h}^{n+1}\right|^{2} \mathrm{~d} x+\frac{3}{4} \Sigma_{i} \varepsilon \beta \int_{\Omega}\left|\nabla \tilde{c}_{i h}^{n+1}\right|^{2} \mathrm{~d} x-\int_{\Omega} \mu_{i h}^{n+1} \tilde{c}_{i h}^{n+1} \mathrm{~d} x=0 .
$$

This is equivalent to:

$$
\int_{\Omega} M_{0 h}^{n} \Delta t\left|\nabla \mu_{i h}^{n+1}\right|^{2} \mathrm{~d} x+\frac{3}{4} \Sigma_{i}^{2} \varepsilon \beta \int_{\Omega}\left|\nabla \tilde{c}_{i h}^{n+1}\right|^{2} \mathrm{~d} x=0
$$


Since the mobility satisfies (1.10), we get: $\nabla \mu_{i h}^{n+1}=\nabla \tilde{c}_{i h}^{n+1}=0$. Hence, $\tilde{c}_{i h}^{n+1}$ and $\mu_{i h}^{n+1}$ are constant. Putting these constants in (4.9) readily leads to $\left(\tilde{c}_{i h}^{n+1}, \mu_{i h}^{n+1}\right)=(0,0)$ and gives the claim.

\subsection{Proof of Theorem $\mathbf{2 . 1 0}$}

\subsubsection{Bounds on discrete solutions}

Inequality (2.26) enables to obtain bounds on the discrete solutions: we can prove a bound in the discrete $\mathrm{L}^{\infty}\left(0, t_{f}, \mathrm{H}^{1}(\Omega)\right)$ norm for the order parameter, in the discrete $\mathrm{L}^{2}\left(0, t_{f}, \mathrm{H}^{1}(\Omega)\right)$ norm for the chemical potentials and in the discrete $\mathrm{L}^{2}\left(0, t_{f},\left(\mathrm{H}^{1}(\Omega)\right)^{\prime}\right)$ norm for the discrete time derivative of the order parameters. Moreover, the presence of numerical diffusion terms in the estimate (2.26) enables to prove that the discrete time derivatives of the order parameters grow at most as $\frac{1}{\sqrt{\Delta t}}$ in the $\mathrm{L}^{2}\left(0, t_{f}, \mathrm{H}^{1}(\Omega)\right)$ norm.

Proposition 4.2. Assume that assumptions of the existence Theorem 2.9 are satisfied. Then, there exists $h_{0}>0$ and positive constants $K_{1}, K_{2}$, independent of $\Delta t$ and $h$ such that, for all $h \leqslant h_{0}$, we have

$$
\begin{aligned}
& \left(\sup _{n \leqslant N}\left|\mathbf{c}_{h}^{n}\right|_{\left(\mathrm{H}^{1}(\Omega)\right)^{3}}\right)+\left(\sum_{n=0}^{N-1} \Delta t \sum_{i=1}^{3}\left|\mu_{i h}^{n+1}\right|_{\mathrm{H}^{1}(\Omega)}^{2}\right) \leqslant K_{1}, \\
& \left(\sum_{n=0}^{N-1} \Delta t \sum_{i=1}^{3}\left|\frac{c_{i h}^{n+1}-c_{i h}^{n}}{\Delta t}\right|_{\left(\mathrm{H}^{1}(\Omega)\right)^{\prime}}^{2}\right)+\Delta t\left(\sum_{n=0}^{N-1} \Delta t \sum_{i=1}^{3}\left|\frac{c_{i h}^{n+1}-c_{i h}^{n}}{\Delta t}\right|_{\mathrm{H}^{1}(\Omega)}^{2}\right) \leqslant K_{2} .
\end{aligned}
$$

Proof. Let $\Sigma_{m}=\min _{i=1,2,3}\left|\Sigma_{i}\right|$ and $\Sigma_{M}=\max _{i=1,2,3}\left|\Sigma_{i}\right|$.

(i) The discrete energy estimate (2.26), gives in particular an uniform bound on the discrete total energy:

$$
\forall n \in \llbracket 0, N \rrbracket, \mathcal{F}_{\boldsymbol{\Sigma}, \varepsilon}^{\text {triph }}\left(\mathbf{c}_{h}^{n}\right) \leqslant \mathcal{F}_{\boldsymbol{\Sigma}, \varepsilon}^{\text {triph }}\left(\mathbf{c}_{h}^{0}\right)
$$

Furthermore, thanks to the polynomial growth assumption (1.11) of $F$, the initial energy $\mathcal{F}_{\boldsymbol{\Sigma}, \varepsilon}^{\text {triph }}\left(\mathbf{c}_{h}^{0}\right)$ can be bounded independently of $h$ :

$$
\mathcal{F}_{\Sigma, \varepsilon}^{\operatorname{triph}}\left(\mathbf{c}_{h}^{0}\right) \leqslant B_{1}\left(|\Omega|+\left|\mathbf{c}_{h}^{0}\right|_{L^{p}}^{p}\right)+\Sigma_{M}\left|\mathbf{c}_{h}^{0}\right|_{\mathrm{H}^{1}}^{2} \leqslant B_{1}\left(|\Omega|+\left|\mathbf{c}^{0}\right|_{\mathrm{H}^{1}}^{p}\right)+\Sigma_{M}\left|\mathbf{c}^{0}\right|_{\mathrm{H}^{1}}^{2}:=K_{0} .
$$

Since $F$ is non negative and by using Proposition 1.1, the bound (4.10) gives in particular,

$$
\forall n \in \llbracket 0, N \rrbracket, \quad \int_{\Omega} \sum_{i=1}^{3}\left|\nabla c_{i h}^{n}\right|^{2} \mathrm{~d} x \leqslant \frac{8}{3 \varepsilon \underline{\Sigma}} K_{0} .
$$

Moreover, the discrete form of the conservation of the volume (2.9) leads to

$$
\forall n \in \mathbb{N}, \quad\left|m\left(c_{i h}^{n}\right)\right| \leqslant|\Omega|^{-\frac{1}{2}}\left|c_{i h}^{0}\right|_{\mathrm{L}^{2}} \leqslant|\Omega|^{-\frac{1}{2}}\left|c_{i}^{0}\right|_{\mathrm{H}^{1}} .
$$

Hence, using (4.12), (4.13) and the Poincaré inequality (4.1), we find that

$$
\forall n \in \llbracket 0, N \rrbracket, \quad\left|\mathbf{c}_{h}^{n}\right|_{\mathrm{H}^{1}(\Omega)} \leqslant C_{p}\left(\frac{16}{3 \varepsilon \Sigma_{m}} K_{0}+\frac{2}{|\Omega|} \sum_{i=1}^{3}\left|c_{i}^{0}\right|_{\mathrm{H}^{1}}^{2}\right)^{\frac{1}{2}}:=K_{1}^{\prime} .
$$


(ii) Now we add the equations (2.26) for $n$ between 0 and $N-1$ :

$$
\begin{aligned}
& \mathcal{F}_{\Sigma, \varepsilon}^{\text {triph }}\left(\mathbf{c}_{h}^{N}\right)-\mathcal{F}_{\boldsymbol{\Sigma}, \varepsilon}^{\text {triph }}\left(\mathbf{c}_{h}^{0}\right) \\
&+C\left[\sum_{n=0}^{N-1} \Delta t \sum_{i=1}^{3} \int_{\Omega} \frac{M_{0 h}^{n+\alpha}}{\Sigma_{i}}\left|\nabla \mu_{i h}^{n+1}\right|^{2} \mathrm{~d} x+\frac{3}{8}(2 \beta-1) \varepsilon \int_{\Omega} \sum_{n=0}^{N-1} \sum_{i=1}^{3} \Sigma_{i}\left|\nabla c_{i h}^{n+1}-\nabla c_{i h}^{n}\right|^{2} \mathrm{~d} x\right] \leqslant 0 .
\end{aligned}
$$

Since $F$ is non negative and the mobility is bounded from below, (4.15) gives in particular

$$
\sum_{n=0}^{N-1} \Delta t \sum_{i=1}^{3} \int_{\Omega}\left|\nabla \mu_{i h}^{n+1}\right|^{2} \mathrm{~d} x \leqslant \frac{2 \Sigma_{M}}{M_{1}} K_{0} .
$$

Let $\theta$ be a non negative given function in $\mathrm{H}^{1}(\Omega)$ with compact support in $\Omega$. We denote by $\theta_{h}$ its $\mathrm{H}^{1}$-projection on $\mathcal{V}_{h D, 0}^{c}$ and we take $\nu_{h}^{c}=\theta_{h}$ as a test function in the second equation of (2.8). We get

$$
|\Omega| m\left(\mu_{i h}^{n+1} \theta_{h}\right)=\int_{\Omega} D_{i}^{F}\left(\mathbf{c}_{h}^{n}, \mathbf{c}_{h}^{n+1}\right) \theta_{h} \mathrm{~d} x+\int_{\Omega} \frac{3}{4} \Sigma_{i} \varepsilon\left[(1-\beta) \nabla c_{i h}^{n}+\beta \nabla c_{i h}^{n+1}\right] \cdot \nabla \theta_{h} \mathrm{~d} x .
$$

Hence, we deduce that

$$
\begin{aligned}
|\Omega|\left|m\left(\mu_{i h}^{n+1} \theta_{h}\right)\right| \leqslant & \frac{4 \Sigma_{T}}{\varepsilon} \sum_{j \neq i}\left(\frac{1}{\left|\Sigma_{j}\right|}\left(\int_{\Omega}\left|d_{i}^{F}\left(\mathbf{c}_{h}^{n}, \mathbf{c}_{h}^{n+1}\right)\right|\left|\theta_{h}\right| \mathrm{d} x+\int_{\Omega}\left|d_{j}^{F}\left(\mathbf{c}_{h}^{n}, \mathbf{c}_{h}^{n+1}\right)\right|\left|\theta_{h}\right| \mathrm{d} x\right)\right) \\
& +\frac{3}{4}\left|\Sigma_{i}\right| \varepsilon\left[(1-\beta) \int_{\Omega}\left|\nabla c_{i h}^{n}\right|\left|\nabla \theta_{h}\right| \mathrm{d} x+\beta \int_{\Omega}\left|\nabla c_{i h}^{n+1}\right|\left|\nabla \theta_{h}\right| \mathrm{d} x\right] .
\end{aligned}
$$

The first term can be bounded as follows (by using (4.14)):

$$
\begin{aligned}
\int_{\Omega}\left|d_{k}^{F}\left(\mathbf{c}_{h}^{n}, \mathbf{c}_{h}^{n+1}\right)\right|\left|\theta_{h}\right| \mathrm{d} x & \leqslant B_{1}\left(1+\left|\mathbf{c}_{h}^{n+1}\right|_{\mathrm{L}^{6}(\Omega)}^{p-1}+\left|\mathbf{c}_{h}^{n}\right|_{\mathrm{L}^{6}(\Omega)}^{p-1}\right)\left|\theta_{h}\right|_{\mathrm{L}^{\frac{6}{7-p}}(\Omega)} \\
& \leqslant B_{1} C_{S, 6}^{2}\left(1+\left|\mathbf{c}_{h}^{n+1}\right|_{\mathrm{H}^{1}(\Omega)}^{p-1}+\left|\mathbf{c}_{h}^{n}\right|_{\mathrm{H}^{1}(\Omega)}^{p-1}\right)\left|\theta_{h}\right|_{\mathrm{H}^{1}(\Omega)} \\
& \leqslant 2 B_{1} C_{S, 6}^{2}\left(K_{1}^{\prime}\right)^{p-1}|\theta|_{\mathrm{H}^{1}(\Omega)},
\end{aligned}
$$

and we get

$$
\begin{aligned}
\left|m\left(\mu_{i h}^{n+1} \theta_{h}\right)\right| \leqslant \frac{1}{|\Omega|} & \frac{16 \Sigma_{T}}{\varepsilon\left|\Sigma_{j}\right|}\left(2 B_{1} C_{S, 6}^{2}\left(K_{1}^{\prime}\right)^{p-1}|\theta|_{\mathrm{H}^{1}(\Omega)}\right) \\
& +\frac{3}{4}\left|\Sigma_{i}\right| \varepsilon\left[(1-\beta)\left|c_{i h}^{n}\right|_{\mathrm{H}^{1}(\Omega)}\left|\theta_{h}\right|_{\mathrm{H}^{1}(\Omega)}+\beta\left|c_{i h}^{n+1}\right|_{\mathrm{H}^{1}(\Omega)}\left|\theta_{h}\right|_{\mathrm{H}^{1}(\Omega)}\right]:=M_{1}^{\theta} .
\end{aligned}
$$

Finally, we readily find

$$
\left|m\left(\mu_{i h}^{n+1} \theta\right)\right| \leqslant \frac{1}{|\Omega|} \int_{\Omega}\left|\mu_{i h}^{n+1}\right|\left|\theta-\theta_{h}\right| \mathrm{d} x+\left|m\left(\mu_{i h}^{n+1} \theta_{h}\right)\right| \leqslant \frac{1}{|\Omega|}\left|\mu_{i h}^{n+1}\right|_{\mathrm{H}^{1}(\Omega)}\left|\theta-\theta_{h}\right|_{\mathrm{L}^{2}(\Omega)}+M_{1}^{\theta},
$$

and the Poincaré inequality (4.1) yields to

$$
\left[1-\frac{C_{p, \theta}}{|\Omega|}\left|\theta-\theta_{h}\right|_{\mathrm{L}^{2}(\Omega)}\right]\left|\mu_{i h}^{n+1}\right|_{\mathrm{H}^{1}(\Omega)} \leqslant C_{p, \theta}\left[\left|\nabla \mu_{i h}^{n+1}\right|_{\mathrm{L}^{2}(\Omega)}+M_{1}^{\theta}\right] .
$$


Owing to (2.5), we can take $h_{0}$ such that for all $h \leqslant h_{0}$, we have $C_{p, \theta}\left|\theta-\theta_{h}\right|_{\mathrm{L}^{2}(\Omega)} \leqslant \frac{1}{2}|\Omega|$. We can conclude by using (4.16) that, for all $h \leqslant h_{0}$,

$$
\sum_{n=0}^{N-1} \Delta t \sum_{i=1}^{3}\left|\mu_{i h}^{n+1}\right|_{\mathrm{H}^{1}(\Omega)}^{2} \leqslant 8 C_{p, \theta}^{2}\left[\frac{2 \Sigma_{M}}{M_{1}} K_{0}+\left(M_{1}^{\theta}\right)^{2}\right]:=K_{1}^{\prime \prime}
$$

(iv) From (4.11) and (4.15), we obtain

$$
\frac{3}{8}(2 \beta-1) C \varepsilon \int_{\Omega} \sum_{n=0}^{N-1} \sum_{i=1}^{3} \Sigma_{i}\left|\nabla c_{i h}^{n+1}-\nabla c_{i h}^{n}\right|^{2} \mathrm{~d} x \leqslant K_{0} .
$$

Defining $K_{2}^{\prime}=\frac{8 C_{p}^{2}}{3(2 \beta-1) \underline{\Sigma} \varepsilon} K_{0}$, using Proposition 1.1, the Poincaré inequality and the volume conservation property (4.13), we finally get

$$
\sum_{n=0}^{N-1} \Delta t \sum_{i=1}^{3}\left|\frac{c_{i h}^{n+1}-c_{i h}^{n}}{\Delta t}\right|_{\mathrm{H}^{1}(\Omega)}^{2} \leqslant \frac{K_{2}^{\prime}}{\Delta t} .
$$

(v) Let $\nu \in \mathrm{H}^{1}(\Omega)$. Denote by $\nu_{h}^{\mu}$ the $\mathrm{L}^{2}$-projection of $\nu$ in $\mathcal{V}_{h}^{\mu}$. Owing to (2.6), we have $\left|\nu_{h}^{\mu}\right|_{\mathrm{H}^{1}(\Omega)} \leqslant$ $C|\nu|_{\mathrm{H}^{1}(\Omega)}$. By using the first equation of (2.8), we obtain

$$
\int_{\Omega} \frac{c_{i h}^{n+1}-c_{i h}^{n}}{\Delta t} \nu_{h}^{\mu} \mathrm{d} x=-\int_{\Omega} \frac{M_{0 h}^{n+\alpha}}{\Sigma_{i}} \nabla \mu_{i h}^{n+1} \cdot \nabla \nu_{h}^{\mu} \mathrm{d} x .
$$

Hence, we find

$$
\left|\left(\frac{c_{i h}^{n+1}-c_{i h}^{n}}{\Delta t}, \nu\right)_{\mathrm{L}^{2}(\Omega)}\right|=\left|\left(\frac{c_{i h}^{n+1}-c_{i h}^{n}}{\Delta t}, \nu_{h}^{\mu}\right)_{\mathrm{L}^{2}(\Omega)}\right| \leqslant \frac{M_{2} C}{\Sigma_{m}}\left|\nabla \mu_{i h}^{n+1}\right|_{\mathrm{L}^{2}(\Omega)}|\nu|_{\mathrm{H}^{1}(\Omega)} .
$$

Since this inequality holds for all $\nu \in \mathrm{H}^{1}(\Omega)$, we have

$$
\left|\frac{c_{i h}^{n+1}-c_{i h}^{n}}{\Delta t}\right|_{\left(\mathrm{H}^{1}(\Omega)\right)^{\prime}}=\sup _{\nu \in \mathrm{H}^{1}(\Omega)} \frac{\left|\left(\frac{c_{i h}^{n+1}-c_{i h}^{n}}{\Delta t}, \nu\right)_{\mathrm{L}^{2}(\Omega)}\right|}{|\nu|_{\mathrm{H}^{1}(\Omega)}} \leqslant \frac{M_{2} C}{\Sigma_{m}}\left|\nabla \mu_{i h}^{n+1}\right|_{\mathrm{L}^{2}(\Omega)},
$$

and thus,

$$
\sum_{n=0}^{N-1} \Delta t \sum_{i=1}^{3}\left|\frac{c_{i h}^{n+1}-c_{i h}^{n}}{\Delta t}\right|_{\left(\mathrm{H}^{1}(\Omega)\right)^{\prime}}^{2} \leqslant\left(\frac{M_{2} C}{\Sigma_{m}}\right)^{2} K_{1}^{\prime \prime}:=K_{2}^{\prime \prime} .
$$

\subsubsection{Estimates of remainders}

The bounds established in Proposition 4.2 and compactness arguments enable to extract convergent subsequences from a given sequence of approximate solutions. Then, it remains to prove that the limit we obtain is a weak solution of the three-phase Cahn-Hilliard model (1.3). Thus, the first step is to specify the link between equations satisfied by the approximate solutions and those satisfied by the weak solution of (1.3).

The following proposition gives estimates on the remainder terms due to the time discretization. 
Proposition 4.3. Let $\tau \in \mathrm{C}_{0}^{\infty}(] 0, t_{f}[), \nu_{h}^{c} \in \mathcal{V}_{D h, 0}^{c}$ and $\nu_{h}^{\mu} \in \mathcal{V}_{h}^{\mu}$. The sequences $\left(\mathbf{c}_{h}^{N}\right)_{N \in \mathbb{N}}$ and $\left(\boldsymbol{\mu}_{h}^{N}\right)_{N \in \mathbb{N}}$ satisfy the following equations,

$$
\left\{\begin{array}{c}
\int_{0}^{t_{f}}\left(\int_{\Omega} \frac{\mathrm{d} c_{i h}^{N}}{\mathrm{~d} t}(t, x) \nu_{h}^{\mu}(x) \mathrm{d} x\right) \tau(t) \mathrm{d} t=-\int_{0}^{t_{f}}\left(\int_{\Omega} \frac{M_{0 h}^{N+\alpha}}{\Sigma_{i}} \nabla \mu_{i h}^{N}(t, x) \cdot \nabla \nu_{h}^{\mu}(x) \mathrm{d} x\right) \tau(t) \mathrm{d} t \\
\int_{0}^{t_{f}}\left(\int_{\Omega} \mu_{i h}^{N}(t, x) \nu_{h}^{c}(x) \mathrm{d} x\right) \tau(t) \mathrm{d} t=\int_{0}^{t_{f}}\left(\int_{\Omega} f_{i}^{F}\left(\mathbf{c}_{h}^{N}(t, x)\right) \nu_{h}^{c}(x) \mathrm{d} x\right) \tau(t) \mathrm{d} t \\
+\int_{0}^{t_{f}}\left(\int_{\Omega} \frac{3}{4} \Sigma_{i} \varepsilon \nabla c_{i h}^{N}(t, x) \cdot \nabla \nu_{h}^{c}(x) \mathrm{d} x\right) \tau(t) \mathrm{d} t+R_{i 1}\left(\nabla \nu_{h}^{c}, \Delta t\right)+R_{i 2}\left(\nu_{h}^{c}, \Delta t\right)
\end{array}\right.
$$

where $M_{0 h}^{N+\alpha}=M_{0}\left((1-\alpha) \underline{\mathbf{c}}_{h}^{N}+\alpha \overline{\mathbf{c}}_{h}^{N}\right)$ and the remainder terms $R_{i 1}$ and $R_{i 2}$ satisfy the following estimates: there exists two constants $K_{3}$ and $K_{4}$ independent of $h$ and $\Delta t$ such that, for all $i \in\{1,2,3\}$,

$$
\begin{aligned}
\left|R_{i 1}\left(\nu_{h}^{c}, \Delta t\right)\right| & \leqslant K_{3}\left|\nu_{h}^{c}\right|_{\mathrm{H}^{1}(\Omega)} \sqrt{\Delta t}, \\
\left|R_{i 2}\left(\nabla \nu_{h}^{c}, \Delta t\right)\right| & \leqslant K_{4}\left|\nabla \nu_{h}^{c}\right|_{\mathrm{L}^{2}(\Omega)} \Delta t .
\end{aligned}
$$

Proof. We extend the function $\tau$ on $\mathbb{R}$ by 0 . The first equation of (4.17) is readily obtained from the first equation of (2.8) by using Definitions (2.24), (2.23), (2.22), (2.25) of $\mathbf{c}_{h}^{N}, \overline{\mathbf{c}}_{h}^{N}, \underline{\mathbf{c}}_{h}^{N}$ and $\boldsymbol{\mu}_{h}^{N}$. Furthermore, multiplying the second equation of $(2.8)$ by the function $\tau$ and integrating on the interval $\left[0, t_{f}\right]$ yields the second equation of (4.17) with

$$
\begin{aligned}
& R_{i 1}=\sum_{n=0}^{N-1} \int_{t_{n}}^{t_{n+1}}\left(\int_{\Omega}\left[D_{i}^{F}\left(\mathbf{c}_{h}^{n}(x), \mathbf{c}_{h}^{n+1}(x)\right)-D_{i}^{F}\left(\mathbf{c}_{h}^{N}(t, x), \mathbf{c}_{h}^{N}(t, x)\right)\right] \nu_{h}^{c}(x) \mathrm{d} x\right) \tau(t) \mathrm{d} t, \\
& R_{i 2}=\sum_{n=0}^{N-1} \int_{t_{n}}^{t_{n+1}}\left(\int_{\Omega} \frac{3}{4} \Sigma_{i} \varepsilon\left[(1-\beta) \nabla c_{i h}^{n}(x)+\beta \nabla c_{i h}^{n+1}(x)-\nabla c_{i h}^{N}(t, x)\right] \cdot \nabla \nu_{h}^{c}(x) \mathrm{d} x\right) \tau(t) \mathrm{d} t .
\end{aligned}
$$

Notice that we use here the consistency assumption (2.3) which implies that

$$
D_{i}^{F}\left(\mathbf{c}_{h}^{N}(t, x), \mathbf{c}_{h}^{N}(t, x)\right)=f_{i}^{F}\left(\mathbf{c}_{h}^{N}(t, x)\right) .
$$

It remains to prove that $R_{i 1}$ and $R_{i 2}$ satisfy the bounds (4.18) and (4.19).

(i) The bound for $R_{i 1}$ is based on the assumptions (2.20) which implies that there exists a constant $T_{1}$ such that for all $(\mathbf{a}, \mathbf{b}) \in \mathcal{S}^{2}$, for all $\lambda \in[0,1]$,

$$
\left|d_{k}(\mathbf{a}, \mathbf{b})-\partial_{k} F(\lambda \mathbf{a}+(1-\lambda) \mathbf{b})\right| \leqslant T_{1}|\mathbf{b}-\mathbf{a}|\left(1+|\mathbf{b}|^{p-2}+|\mathbf{a}|^{p-2}\right) .
$$

Thus, since $2 \leqslant p \leqslant 6$, we have $1 \leqslant \frac{6}{7-p} \leqslant 6, \frac{6}{p-2} \geqslant 0$ and $\frac{7-p}{6}+\frac{p-2}{6}+\frac{1}{6}=1$ and we can apply the Hölder inequality, to obtain that there exists a constant $T_{2}$ such that

$$
\left|\int_{\Omega}\left(d_{k}^{F}\left(\mathbf{c}_{h}^{n}(x), \mathbf{c}_{h}^{n+1}(x)\right)-\partial_{k} F\left(\mathbf{c}_{h}^{N}(t, x)\right)\right) \nu_{h}^{c}(x) \mathrm{d} x\right| \leqslant T_{2}\left(1+2 K_{1}^{p-2}\right)\left|\nu_{h}^{c}\right|_{\mathrm{H}^{1}(\Omega)}\left|\mathbf{c}_{h}^{n+1}-\mathbf{c}_{h}^{n}\right|_{\mathrm{H}^{1}(\Omega)} .
$$

Since $R_{i 1}$ can be written as follows

$$
\begin{aligned}
R_{i 1}=\frac{4 \Sigma_{T}}{\varepsilon} \sum_{j \neq i} \frac{1}{\Sigma_{j}} \sum_{n=0}^{N-1} \int_{t_{n}}^{t_{n+1}} \int_{\Omega} & {\left[\left(d_{i}^{F}\left(\mathbf{c}_{h}^{n}(x), \mathbf{c}_{h}^{n+1}(x)\right)-\partial_{i} F\left(\mathbf{c}_{h}^{N}(t, x)\right)\right)\right.} \\
& \left.-\left(d_{j}^{F}\left(\mathbf{c}_{h}^{n}(x), \mathbf{c}_{h}^{n+1}(x)\right)-\partial_{j} F\left(\mathbf{c}_{h}^{N}(t, x)\right)\right)\right] \nu_{h}^{c}(x) \mathrm{d} x \tau(t) \mathrm{d} t ;
\end{aligned}
$$


we have

$$
\begin{aligned}
& \left|\sum_{n=0}^{N-1} \int_{t_{n}}^{t_{n+1}} \int_{\Omega}\left(d_{k}^{F}\left(\mathbf{c}_{h}^{n}(x), \mathbf{c}_{h}^{n+1}(x)\right)-\partial_{k} F\left(\mathbf{c}_{h}^{N}(t, x)\right)\right) \nu_{h}^{c}(x) \mathrm{d} x \tau(t) \mathrm{d} t\right| \\
& \quad \leqslant T_{2}\left(1+2 K_{1}^{p-2}\right)\left|\nu_{h}^{c}\right|_{\mathrm{H}^{1}(\Omega)}\left(\sup _{t \in\left[0, t_{f}\right]}|\tau(t)|\right) \Delta t\left(\sum_{n=0}^{N-1} \Delta t\left|\frac{\mathbf{c}_{h}^{n+1}-\mathbf{c}_{h}^{n}}{\Delta t}\right|_{\mathrm{H}^{1}(\Omega)}^{2}\right)^{\frac{1}{2}} .
\end{aligned}
$$

In conclusion, using the third bound of Theorem 4.2, we get

$$
\left|R_{i 1}\right| \leqslant T_{2} K_{2}\left(1+2 K_{1}^{p-2}\right)|\tau|_{L^{\infty}\left(\left[0, t_{f}\right]\right)}\left|\nu_{h}^{c}\right|_{\mathrm{H}^{1}(\Omega)} \Delta t^{\frac{1}{2}}
$$

Hence, estimate (4.18) holds with $K_{3}:=T_{2} K_{2}\left(1+2 K_{1}^{p-2}\right)|\tau|_{L^{\infty}\left(\left[0, t_{f}\right]\right)}$.

(ii) A renumbering of the terms yields

$$
\begin{aligned}
& R_{i 2}=\sum_{n=0}^{N-1} \int_{t_{n}}^{t_{n+1}}\left(\int_{\Omega} \frac{3}{4} \Sigma_{i} \varepsilon\left[\left(\beta-\frac{t-t_{n}}{\Delta t}\right)\left(\nabla c_{i h}^{n+1}(x)-\nabla c_{i h}^{n}(x)\right)\right] \cdot \nabla \nu_{h}^{c}(x) \mathrm{d} x\right) \tau(t) \mathrm{d} t \\
& =\frac{3}{4} \Sigma_{i} \varepsilon \sum_{n=0}^{N-1} \int_{0}^{1}\left(\int_{\Omega} \Delta t\left[(\beta-u)\left(\nabla c_{i h}^{n+1}(x)-\nabla c_{i h}^{n}(x)\right)\right] \cdot \nabla \nu_{h}^{c}(x) \mathrm{d} x\right) \tau((n+u) \Delta t) \mathrm{d} u \\
& =\frac{3}{4} \Sigma_{i} \varepsilon \sum_{n=0}^{N} \Delta t\left(\int_{\Omega} \nabla c_{i h}^{n}(x) \cdot \nabla \nu_{h}^{c}(x) \mathrm{d} x\right)(\int_{0}^{1}(\beta-u) \underbrace{(\tau((n-1+u) \Delta t)-\tau((n+u) \Delta t))}_{\leqslant\left.\Delta t \tau^{\prime}\right|_{L^{\infty}(\mathbb{R})}} \mathrm{d} u)
\end{aligned}
$$

and by Theorem 4.2 , we obtain

$$
\left|R_{i 2}\right| \leqslant \frac{3}{4} \Sigma_{M} \varepsilon(N+1) \Delta t K_{1}\left|\nabla \nu_{h}^{c}(x)\right|_{L^{2}(\Omega)} \Delta t\left|\tau^{\prime}\right|_{L^{\infty}(\mathbb{R})} \leqslant \frac{3}{4} \Sigma_{M} \varepsilon 2 t_{f}\left|\nabla \nu_{h}^{c}(x)\right|_{L^{2}(\Omega)} \Delta t\left|\tau^{\prime}\right|_{L^{\infty}(\mathbb{R})} .
$$

Hence, estimate (4.19) holds with $K_{4}=\frac{3}{2} K_{1} t_{f} \Sigma_{M} \varepsilon\left|\tau^{\prime}\right|_{L^{\infty}(\mathbb{R})}$.

In order to be able to show the convergence when the time step and the mesh size tend to zero, we have also to estimate the remainders due to the space discretization.

Proposition 4.4. Let $\tau \in \mathrm{C}_{0}^{\infty}(] 0, t_{f}[), \nu^{c} \in \mathcal{V}_{D, 0}^{c}$ and $\nu^{\mu} \in \mathcal{V}^{\mu}$. The sequences $\left(\mathbf{c}_{h}^{N}\right)_{N \in \mathbb{N}}$ and $\left(\boldsymbol{\mu}_{h}^{N}\right)_{N \in \mathbb{N}}$ satisfy the following equations,

$$
\begin{gathered}
\int_{0}^{t_{f}}\left(\int_{\Omega} \frac{\mathrm{d} c_{i h}^{N}}{\mathrm{~d} t}(t, x) \nu^{\mu}(x) \mathrm{d} x\right) \tau(t) \mathrm{d} t=-\int_{0}^{t_{f}}\left(\int_{\Omega} \frac{M_{0 h}^{N+\alpha}}{\Sigma_{i}} \nabla \mu_{i h}^{N}(t, x) \cdot \nabla \nu^{\mu}(x) \mathrm{d} x\right) \tau(t) \mathrm{d} t+R_{i 3}(h, \Delta t) \\
\int_{0}^{t_{f}}\left(\int_{\Omega} \mu_{i h}^{N}(t, x) \nu^{c}(x) \mathrm{d} x\right) \tau(t) \mathrm{d} t=\int_{0}^{t_{f}}\left(\int_{\Omega} f_{i}^{F}\left(\mathbf{c}_{h}^{N}(t, x)\right) \nu^{c}(x) \mathrm{d} x\right) \tau(t) \mathrm{d} t \\
\quad+\int_{0}^{t_{f}}\left(\int_{\Omega} \frac{3}{4} \Sigma_{i} \varepsilon \nabla c_{i h}^{N}(t, x) \cdot \nabla \nu^{c}(x) \mathrm{d} x\right) \tau(t) \mathrm{d} t+R_{i 1}(h, \Delta t)+R_{i 2}(h, \Delta t)+R_{i 4}(h, \Delta t)
\end{gathered}
$$


where $R_{i 1}, R_{i 2}, R_{i 3}$ and $R_{i 4}$ satisfy the following estimates: there exist four constants $K_{5}, K_{6}, K_{7}$ and $K_{8}$ independent of $h$ and $\Delta t$ such that,

$$
\begin{aligned}
\left|R_{i 1}(h, \Delta t)\right| & \leqslant K_{5} \Delta t \\
\left|R_{i 2}(h, \Delta t)\right| & \leqslant K_{6} \sqrt{\Delta t} \\
\left|R_{i 3}(h, \Delta t)\right| & \leqslant K_{7} \inf _{\nu_{h}^{\mu} \in \mathcal{V}_{h}^{\mu}}\left|\nu^{\mu}-\nu_{h}^{\mu}\right|_{\mathrm{H}^{1}(\Omega)}, \\
\left|R_{i 4}(h, \Delta t)\right| & \leqslant K_{8} \inf _{\nu_{h}^{c} \in \mathcal{V}_{D h, 0}^{c}}\left|\nu^{c}-\nu_{h}^{c}\right|_{\mathrm{H}^{1}(\Omega)}
\end{aligned}
$$

Proof. Let $\nu_{h}^{c}\left(\right.$ resp. $\left.\nu_{h}^{\mu}\right)$, be the $\mathrm{H}^{1}$-projection of $\nu^{c}\left(\right.$ resp. $\left.\nu^{\mu}\right)$, on $\mathcal{V}_{h D, 0}^{c}\left(\right.$ resp. $\left.\mathcal{V}_{h}^{\mu}\right)$. By using Theorem 4.3 and then denoting by $R_{i 1}(h, \Delta t)$ and $R_{i 2}(h, \Delta t)$ the terms $R_{i 1}\left(\nu_{h}^{c}, \Delta t\right)$ and $R_{i 2}\left(\nabla \nu_{h}^{c}, \Delta t\right)$, we see that the remainder terms $R_{i 3}$ and $R_{i 4}$ are given by

$$
\begin{aligned}
R_{i 3}(h, \Delta t)=\int_{0}^{t_{f}}\left(\int_{\Omega} \frac{\mathrm{d} c_{i h}^{N}}{\mathrm{~d} t}(t, x)\left(\nu^{\mu}(x)-\nu_{h}^{\mu}(x)\right) \mathrm{d} x\right) \tau(t) \mathrm{d} t & \\
& +\int_{0}^{t_{f}}\left(\int_{\Omega} \frac{M_{0 h}^{N+\alpha}}{\Sigma_{i}} \nabla \mu_{i h}^{N}(t, x) \cdot \nabla\left(\nu^{\mu}(x)-\nu_{h}^{\mu}(x)\right) \mathrm{d} x\right) \tau(t) \mathrm{d} t,
\end{aligned}
$$

and

$$
\begin{gathered}
R_{i 4}(h, \Delta t)=\int_{0}^{t_{f}}\left(\int_{\Omega} \mu_{i h}^{N}(t, x)\left(\nu^{c}(x)-\nu_{h}^{c}(x)\right) \mathrm{d} x\right) \tau(t) \mathrm{d} t-\int_{0}^{t_{f}}\left(\int_{\Omega} f_{i}^{F}\left(\mathbf{c}_{h}^{N}(t, x)\right)\left(\nu^{c}(x)-\nu_{h}^{c}(x)\right) \mathrm{d} x\right) \tau(t) \mathrm{d} t \\
-\int_{0}^{t_{f}}\left(\int_{\Omega} \frac{3}{4} \Sigma_{i} \varepsilon \nabla c_{i h}^{N}(t, x) \cdot \nabla\left(\nu^{c}(x)-\nu_{h}^{c}(x)\right) \mathrm{d} x\right) \tau(t) \mathrm{d} t .
\end{gathered}
$$

The bound for $R_{i 1}$ and $R_{i 2}$ readily follows from $\left|\nu_{h}^{c}\right|_{\mathrm{H}^{1}(\Omega)} \leqslant\left|\nu^{c}\right|_{\mathrm{H}^{1}(\Omega)}$. The bound for $R_{i 3}$ is obtained as follows:

$$
\begin{aligned}
\left|R_{i 3}\right| & \leqslant\left|\frac{\mathrm{d} c_{i h}^{N}}{\mathrm{~d} t}\right|_{\mathrm{L}^{2}\left(0, t_{f},\left(\mathrm{H}^{1}(\Omega)\right)^{\prime}\right)}|\tau|_{\mathrm{L}^{2}\left(0, t_{f}\right)}\left|\nu^{\mu}-\nu_{h}^{\mu}\right|_{\mathrm{H}^{1}(\Omega)}+\frac{M_{2}}{\Sigma_{m}}\left|\mu_{i h}^{N}\right|_{\mathrm{L}^{2}\left(0, t_{f}, \mathrm{H}^{1}(\Omega)\right)}|\tau|_{\mathrm{L}^{2}\left(0, t_{f}\right)}\left|\nu^{\mu}-\nu_{h}^{\mu}\right|_{\mathrm{H}^{1}(\Omega)} \\
& \leqslant K_{7}\left|\nu^{\mu}-\nu_{h}^{\mu}\right|_{\mathrm{H}^{1}(\Omega)},
\end{aligned}
$$

with $K_{7}:=\left(\frac{M_{2}}{\Sigma_{m}} \sqrt{K_{1}}+K_{2}\right)|\tau|_{L^{2}\left(0, t_{f}\right)}$, and the bound for $R_{4}$ is deduced from the following inequalities:

$$
\begin{aligned}
\left|R_{i 4}\right| \leqslant & \left|\mu_{i h}^{N}\right|_{\mathrm{L}^{2}\left(0, t_{f}, \mathrm{~L}^{2}(\Omega)\right)}|\tau|_{\mathrm{L}^{2}\left(0, t_{f}\right)}\left|\nu^{c}-\nu_{h}^{c}\right|_{\mathrm{L}^{2}(\Omega)} \\
& +\frac{24 \Sigma_{T}}{\varepsilon \Sigma_{m}} \int_{0}^{t_{f}} B_{1}\left(\left|c_{i h}^{N}(t, \cdot)\right|_{\mathrm{L}^{6}(\Omega)}^{p-1}\left|\nu^{c}-\nu_{h}^{c}\right|_{\mathrm{L}^{\frac{6}{7-p}}(\Omega)}+|\Omega|^{\frac{1}{2}}\left|\nu^{c}-\nu_{h}^{c}\right|_{\mathrm{L}^{2}(\Omega)}\right) \tau(t) \mathrm{d} t \\
& +\frac{3}{4} \Sigma_{M} \varepsilon t_{f}\left|c_{i h}^{N}\right|_{\mathrm{L}^{\infty}\left(0, t_{f}, \mathrm{H}^{1}(\Omega)\right)}|\tau|_{\mathrm{L}^{\infty}\left(0, t_{f}\right)}\left|\nu^{c}-\nu_{h}^{c}\right|_{\mathrm{H}^{1}(\Omega)} \\
\leqslant & \underbrace{\left[\sqrt{K_{1}}|\tau|_{\mathrm{L}^{2}\left(0, t_{f}\right)}+\frac{24 \Sigma_{T}}{\varepsilon \Sigma_{m}} t_{f}|\tau|_{\mathrm{L}^{\infty}\left(0, t_{f}\right)} B_{1}\left(K_{1}^{p-1}+|\Omega|^{\frac{1}{2}}\right)+\frac{3}{4} \Sigma_{M} \varepsilon K_{1}|\tau|_{\mathrm{L}^{\infty}\left(0, t_{f}\right)} t_{f}\right]}_{:=K_{8}}\left|\nu^{c}-\nu_{h}^{c}\right|_{\mathrm{H}^{1}(\Omega)} .
\end{aligned}
$$




\subsubsection{Proof of Theorem 2.10}

Theorem 4.2 readily yields to the following bounds:

$$
\begin{gathered}
\left|\mathbf{c}_{h_{K}}^{N}\right|_{\mathrm{L}^{\infty}\left(0, t_{f},\left(\mathrm{H}^{1}(\Omega)\right)^{3}\right)}+\left|\boldsymbol{\mu}_{h_{K}}^{N}\right|_{\mathrm{L}^{2}\left(0, t_{f},\left(\mathrm{H}^{1}(\Omega)\right)^{3}\right)}^{2}+\left|\frac{\partial \mathbf{c}_{h_{K}}^{N}}{\partial t}\right|_{\mathrm{L}^{2}\left(0, t_{f},\left(\mathrm{H}^{1}(\Omega)\right)^{\prime}\right)}^{2} \leqslant K_{1}+K_{2}, \\
\left|\mathbf{c}_{h_{K}}^{N}-\underline{\mathbf{c}}_{h_{K}}^{N}\right|_{\mathrm{L}^{2}\left(0, t_{f},\left(\mathrm{H}^{1}(\Omega)\right)^{3}\right)}+\left|\mathbf{c}_{h_{K}}^{N}-\overline{\mathbf{c}}_{h_{K}}^{N}\right|_{\mathrm{L}^{2}\left(0, t_{f},\left(\mathrm{H}^{1}(\Omega)\right)^{3}\right)} \leqslant 2 \sqrt{K_{2} \Delta t} .
\end{gathered}
$$

By using estimates (4.22a), we can extract subsequences of $\left(\mathbf{c}_{h_{K}}^{N}\right)_{(N, K)}$ and $\left(\boldsymbol{\mu}_{h_{K}}^{N}\right)_{(N, K)}$ (still denoted by $\left(\mathbf{c}_{h_{K}}^{N}\right)_{(N, K)}$ and $\left.\left(\boldsymbol{\mu}_{h_{K}}^{N}\right)_{(N, K)}\right)$ such that

$$
\begin{array}{cc}
\mathbf{c}_{h_{K}}^{N} \rightarrow \mathbf{c} & \text { in } \mathrm{L}^{\infty}\left(0, t_{f},\left(\mathrm{H}^{1}(\Omega)\right)^{3}\right) \text { weak-*, } \\
\boldsymbol{\mu}_{h_{K}}^{N} \rightarrow \boldsymbol{\mu} & \text { in } \mathrm{L}^{2}\left(0, t_{f},\left(\mathrm{H}^{1}(\Omega)\right)^{3}\right) \text { weak, } \\
\frac{\partial \mathbf{c}_{h_{K}}^{N}}{\partial t} \rightarrow \frac{\partial \mathbf{c}}{\partial t} & \text { in } \mathrm{L}^{2}\left(0, t_{f},\left(\mathrm{H}^{1}(\Omega)\right)^{\prime}\right) \text { weak. }
\end{array}
$$

From estimate (4.22a), we can use the Aubin-Lions-Simon's compactness theorem [32] to obtain, up to a subsequence,

$$
\mathbf{c}_{h_{K}}^{N} \rightarrow \mathbf{c} \quad \text { in } \mathrm{C}^{0}\left(0, t_{f},\left(\mathrm{~L}^{q}(\Omega)\right)^{3}\right) \text { strong, for all } 1 \leqslant q<+\infty \text { if } d=2 \text {, or } 1 \leqslant q<6 \text { if } d=3 .
$$

In particular, (4.26) implies that

$$
\mathbf{c}_{h_{K}}^{N} \rightarrow \mathbf{c} \quad \text { in } \mathrm{L}^{2}\left(0, t_{f},\left(\mathrm{~L}^{2}(\Omega)\right)^{3}\right) \text { strong, }
$$

and then estimate $(4.22 \mathrm{~b})$ leads to

$$
\begin{aligned}
& \underline{\mathbf{c}}_{h_{K}}^{N} \rightarrow \mathbf{c} \quad \text { in } \mathrm{L}^{2}\left(0, t_{f},\left(\mathrm{~L}^{2}(\Omega)\right)^{3}\right) \text { strong } \\
& \overline{\mathbf{c}}_{h_{K}}^{N} \rightarrow \mathbf{c} \quad \text { in } \mathrm{L}^{2}\left(0, t_{f},\left(\mathrm{~L}^{2}(\Omega)\right)^{3}\right) \text { strong. }
\end{aligned}
$$

Let $\tau \in \mathrm{C}_{0}^{\infty}(] 0, t_{f}[), \nu^{c} \in \mathcal{V}_{D, 0}^{c}$ and $\nu^{\mu} \in \mathcal{V}^{\mu}$. We can apply Theorem 4.4 and pass to the limit in (4.21):

(i) Convergences (4.25), (4.24) and (4.23) allow to pass to the limit in the linear terms.

(ii) The terms $R_{i 1}, R_{i 2}, R_{i 3}$ and $R_{i 4}$ tend to 0 thanks to assumptions (2.5).

(iii) Using the fact that the space $\mathrm{C}^{\infty}(\bar{\Omega})$ is dense in $\mathcal{V}^{\mu}=\mathrm{H}^{1}(\Omega)$, that $M_{0}(\mathbf{c}) \in \mathrm{L}^{\infty}(] 0, t_{f}\left[, \mathrm{~L}^{\infty}(\Omega)\right)$ and owing to the convergences (4.24), (4.28) and (4.29), we obtain that for any $\nu^{\mu} \in \mathcal{V}^{\mu}$,

$$
\int_{0}^{t_{f}}\left(\int_{\Omega} \frac{M_{0 h_{K}}^{N+\alpha}}{\Sigma_{i}} \nabla \mu_{i h_{K}}^{N}(t, x) \cdot \nabla \nu^{\mu}(x) \mathrm{d} x\right) \tau(t) \mathrm{d} t \longrightarrow \int_{0}^{t_{f}}\left(\int_{\Omega} \frac{M_{0}(\mathbf{c})}{\Sigma_{i}} \nabla \mu_{i}(t, x) \cdot \nabla \nu^{\mu}(x) \mathrm{d} x\right) \tau(t) \mathrm{d} t .
$$

(iv) The convergence (4.27) and the Lebesgue theorem allow to prove that, up to a subsequence,

$$
\int_{0}^{t_{f}}\left(\int_{\Omega} f_{i}^{F}\left(\mathbf{c}_{h_{K}}^{N}(t, x)\right) \nu^{c}(x) \mathrm{d} x\right) \tau(t) \mathrm{d} t \rightarrow \int_{0}^{t_{f}}\left(\int_{\Omega} f_{i}^{F}(\mathbf{c}(t, x)) \nu^{c}(x) \mathrm{d} x\right) \tau(t) \mathrm{d} t
$$

This shows the existence of a weak solution $(\mathbf{c}, \boldsymbol{\mu})$ to problem (1.3) and the convergences (2.27) and (2.28). 


\section{NUMERICAL EXPERIMENTS}

In this section, we present some numerical experiments in one dimension and two dimensions in order to compare the different time discretizations of the nonlinear terms presented in Section 3. The practical implementation has been performed using the software object-oriented component library PELICANS [29], developed at the "Institut de Radioprotection et de Sûreté Nucléaire (IRSN)" and distributed under the CeCILL-C license agreement (an adaptation of LGPL to the French law).

We use the following notation for the schemes:

- Impl. stands for the implicit discretization (3.4) for the contribution of $F_{0}$, the semi-implicit discretization (3.19) for the contribution of $P$ and $\beta=1$;

- CC. stands for the convex-concave discretization (3.11) for the contribution of $F_{0}$, the semi-implicit discretization (3.19) for the contribution of $P$ and $\beta=1$;

- SImpl. $(\beta)$ stands for the semi-implicit discretization (3.16) for the contribution of $F_{0}$, the semi-implicit discretization (3.19) for the contribution of $P$ and the given value of $\beta$;

- SImpl. stands for $\operatorname{SImpl}(1)$.

In one dimensional test cases, the spatial discretization is performed by using an uniform grid and piecewise linear finite element functions. In two dimensions, in order to limit the computational cost, we use $\mathbb{Q}_{1}$ Lagrange finite element on square local adaptive refined meshes. The adaptation procedures are based on conforming multilevel finite element approximation spaces which are built by refinement or unrefinement of the finite element basis functions instead of cells. All the details about this method and also various examples (in particular, simulations using the Cahn-Hilliard model considered in this article) are described in [9].

The refinement criterion used in those (un-)refinement procedures imposes the value of the smaller diameter $h_{\min }$ of a cell and ensures that refined areas are located in the neighborhood of the interfaces (i.e. where no order parameter is equal to one). In practice, we take $h_{\min }=\frac{\varepsilon}{2}$ to ensure the presence of at least three or four cells in interfaces. We observe that this criterion enables to obtain a sufficient resolution in the interface without increasing too much the computational cost: we performed numerical computations with $h_{\min }=\frac{\varepsilon}{4}$ and we observed very similar results. We do not give more details on spatial discretization issues here since the main goal of this article is to investigate the properties of various time discretization schemes. However, we give some comparison elements in Remark 5.1 between the computations with or without the adaptive refinement algorithm.

In all of the two dimensional test cases, the approximate solutions are visualized through the isolines of the function:

$$
\left(c_{1}, c_{2}, c_{3}\right) \mapsto\left(1-c_{1}\right)\left(1-c_{2}\right)\left(1-c_{3}\right)
$$

which is non zero only in the interface; and figures which represent approximate solutions also show the refined mesh used for the corresponding computation.

For convergence studies, for each scheme, different approximate solutions $\mathbf{c}^{\Delta t_{j}}$ are computed using several time steps $\Delta t_{j}$. Since non trivial analytic solutions of Cahn-Hilliard system (1.3) are not known, we use an approximate solution $\mathbf{c}^{\Delta t_{\text {ref }}}$ obtained with a reference time step $\Delta t_{\text {ref }}$ as a reference solution. Obviously, $\Delta t_{\text {ref }}$ is assumed to be small enough compared to $\Delta t_{j}$. Although the refinement criterion is the same for all computations, the refined grids can slightly differ from a computation to another since time steps are different. However the $\mathrm{L}^{2}$ norm of the error

$$
e_{j}(t)=\left|\mathbf{c}^{\Delta t_{j}}(t, \cdot)-\mathbf{c}^{\Delta t_{\text {ref }}}(t, \cdot)\right|_{\left(\mathrm{L}^{2}(\Omega)\right)^{3}},
$$

at a fixed time $t$, is exactly computed on the uniform grid of size $h_{\min }$ during a post-processing step.

\subsection{Two-phase test cases}

In this subsection, the schemes are compared on two-phase test cases. In other words, the three-phase Problem 2.4 is numerically solved but the third order parameter $c_{3}$ is initialized to zero on the whole domain 


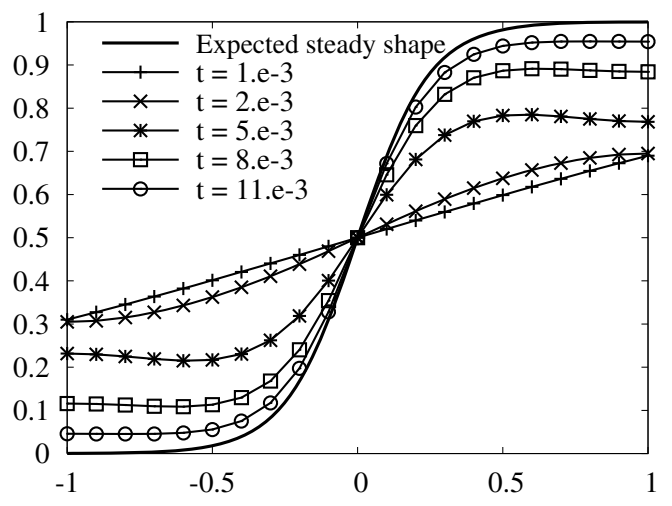

FIGURE 1. Evolution of the first parameter order $c_{1}$ using the Impl. scheme with $\Delta t=10^{-3}$.

so that the two phases in presence are described by the order parameters $c_{1}$ and $c_{2}=1-c_{1}$. The consistency property (see Sects. 1.2 and 3.7) ensures that the order parameter $c_{3}$ will stay at zero all along the simulations and, consequently, the schemes we actually compare are the ones presented in Section 3.7.

Two test cases are given in order to illustrate the two different behaviors of the Cahn-Hilliard system: the first one is the stability of the observed interface thickness close to $\varepsilon$ and the second one is the motion of the interface governed by surface tensions.

\subsubsection{Dynamics of one interface in $1 D$}

The first experiment is performed on the space domain $[-1,1]$ with the following parameters: the interface thickness $\varepsilon=0.5$, a constant mobility $M=8$ and a surface tension between the two present phases $\sigma=1$. We impose Neumann boundary conditions for both order parameters and chemical potentials. The initial data is given by:

$$
c_{1}^{0}(x)=\frac{1}{2}\left(1+\tanh \left(\frac{2 x}{10 \varepsilon}\right)\right), \quad \text { and } \quad c_{2}^{0}(x)=0, \quad \forall x \in[-1,1] .
$$

Figure 1 shows the evolution of the order parameter $c_{1}$ towards the equilibrium shape. We also represent, in this figure, an approximation of the steady solution:

$$
c_{0}(x)=\frac{1}{2}\left(1+\tanh \left(\frac{2 x}{\varepsilon}\right)\right), \quad \forall x \in \mathbb{R},
$$

which is obtained by exactly solving the following interface profile problem on an infinite domain:

$$
\left\{\begin{array}{l}
-\frac{3}{2} \sigma \varepsilon c_{0}^{\prime \prime}(x)+12 \frac{\sigma}{\varepsilon} f^{\prime}\left(c_{0}(x)\right)=0, \quad \forall x \in \mathbb{R}, \\
\lim _{+\infty} c_{0}=1, \quad \lim _{-\infty} c_{0}=0, \quad c_{0}(0)=\frac{1}{2},
\end{array}\right.
$$

where $f$ is the function defined in (3.1).

Figure 2 presents the convergence study. The reference solution is computed using the SImpl.(0.5) scheme with $\Delta t_{\text {ref }}=10^{-8}$. We perform several computations using the different schemes and for each of the following time steps $\Delta t_{j}: 2 \times 10^{-4}, 5 \times 10^{-4}, 10^{-4}, 2 \times 10^{-5}, 5 \times 10^{-5}, 10^{-5}, 10^{-6}$. The $\mathrm{L}^{2}$-norm of the corresponding errors $e_{j}(t)$ at time $t=0.01$ are represented in the picture on the left and the convergence rates of each scheme are given in the table on the right. We observe a first order convergence for the Impl., CC., SImpl. schemes and a (almost) second order convergence for the SImpl.(0.5) scheme. Note also that the CC. scheme is less accurate than the other ones, whereas the SImpl. scheme enables to achieve the same accuracy as the Impl. scheme. 


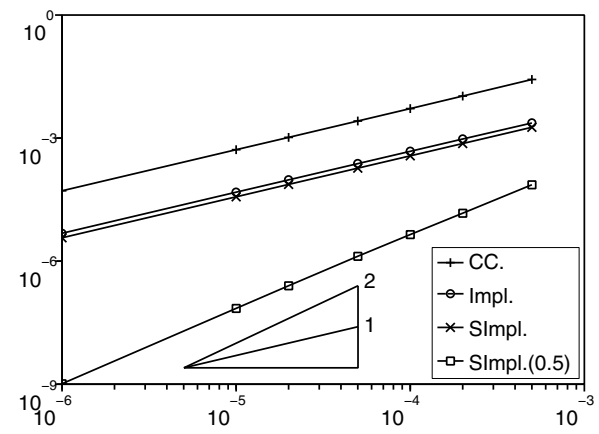

\begin{tabular}{||l|c||}
\hline Scheme & Convergence rate \\
\hline \hline CC. & 1.0 \\
\hline SImpl. & 1.0 \\
\hline Impl. & 1.0 \\
\hline SImpl.(0.5) & 1.8 \\
\hline
\end{tabular}

Figure 2. Errors $e_{j}(t)=\left|\mathbf{c}^{\Delta t_{j}}(t, \cdot)-\mathbf{c}^{\Delta t_{\text {ref }}}(t, \cdot)\right|_{\left(\mathrm{L}^{2}(\Omega)\right)^{3}}$ at time $t=0.01$ as a function of the time step $\Delta t_{j}$ (left) and convergence rates (right) obtained for the different schemes.
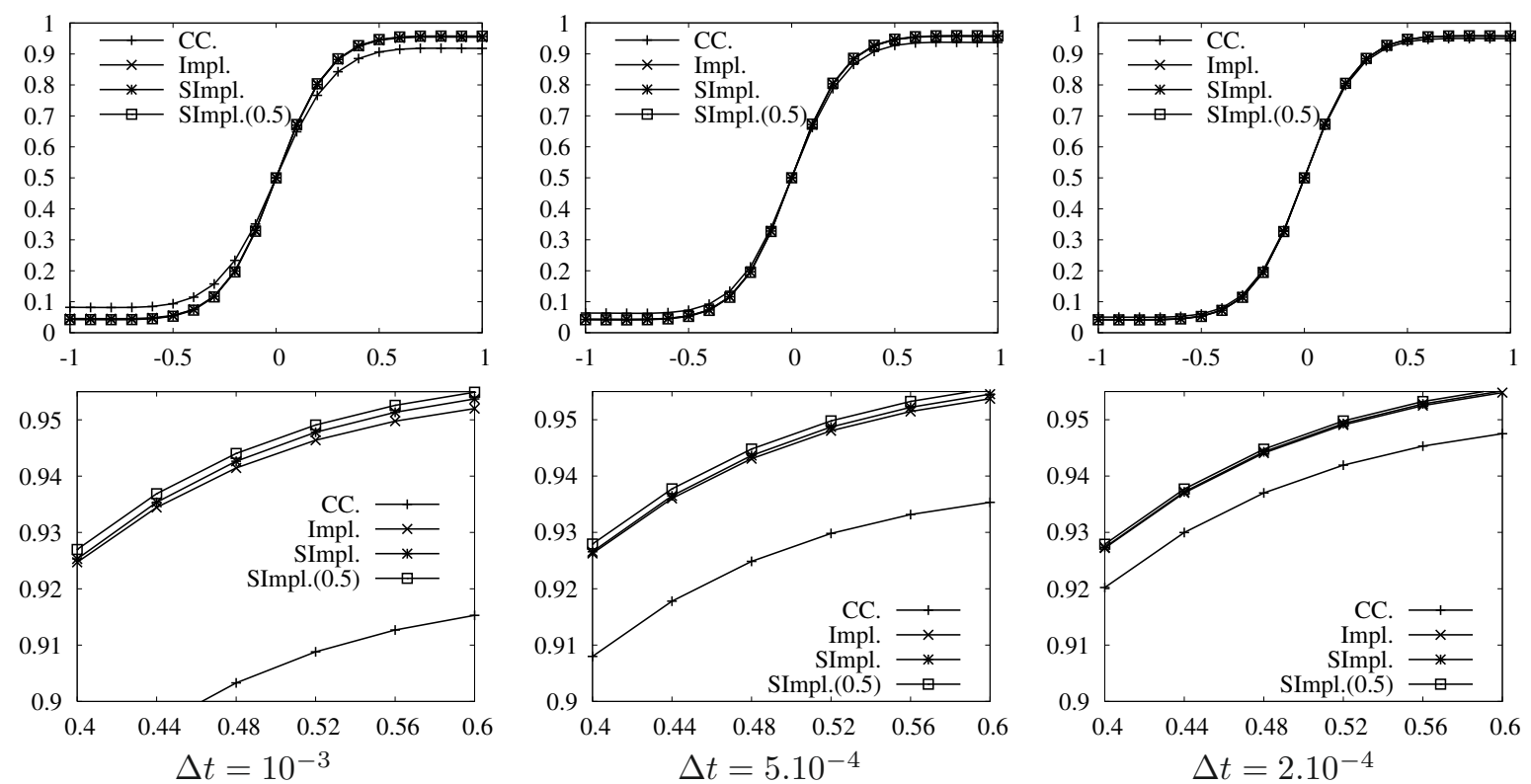

FiguRE 3. First order parameter $c_{1}$ as a function of the space variable $x$ at time $t=0.01$. Top: $x \in[-1,1]$; bottom: $x \in[0.4,0.6]$ (zoom).

The influence of the different schemes on the shape of the solution is illustrated in Figure 3. We represent, for different time steps, the first order parameter $c_{1}$ as a function of the space variable on the whole domain (at the top) and on a zoomed part (at the bottom). The Impl., SImpl, SImpl.(0.5) schemes give very close results whereas the CC. scheme gives a significantly different profile.

\subsubsection{Ellipsoidal bubble - Neumann boundary conditions}

This experiment is performed on the space domain $[-0.2,0.2]^{2}$ with the following parameters: the interface thickness $\varepsilon=0.01$, a constant mobility $M=10^{-4}$ and a surface tension between the two present phases $\sigma=1$. We impose Neumann boundary conditions for both order parameters and chemical potentials. The initial data 

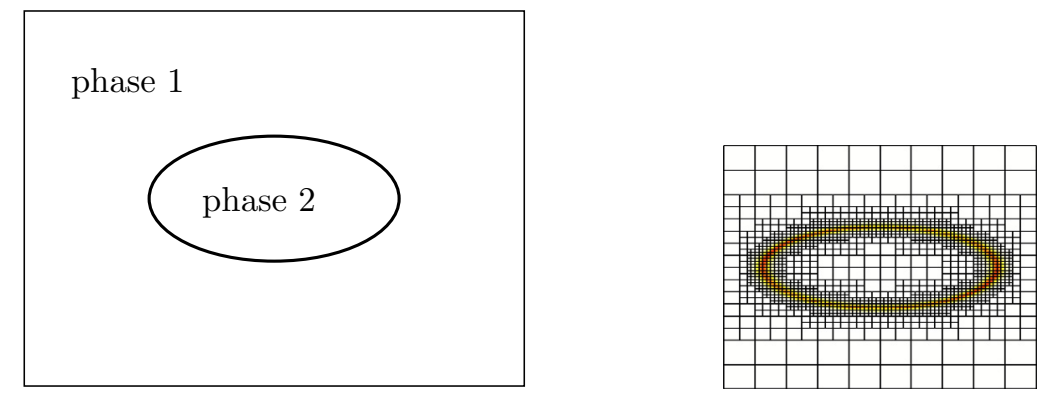

Figure 4. Configuration of test case (left) and initial position of interface (right). (Figure in color available online at http://www.esaim-m2an.org/.)

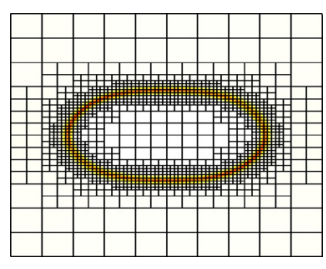

$t=0.8$

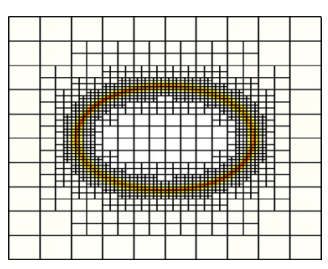

$t=1.8$

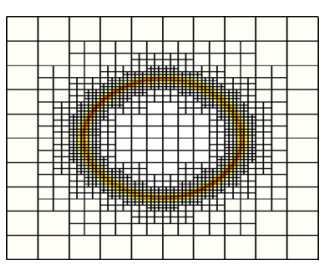

$t=4.8$

Figure 5. Evolution of the interface position using the Impl. scheme with $\Delta t=5 \times 10^{-4}$.

(Figure in color available online at http://www.esaim-m2an.org/.)

is given by:

$$
c_{1}^{0}(x, y)=\frac{1}{2}\left[1+\tanh \left(\frac{2}{\varepsilon}\left[\left(\frac{x^{2}}{a^{2}}+a^{2} y^{2}\right)^{\frac{1}{2}}-0.1\right]\right)\right], \quad c_{2}^{0}(x, y)=0, \quad \forall(x, y) \in[-0.2,0.2]^{2},
$$

where $a=1.5$.

Figure 4 shows the initial configuration on the left and the position of the interfaces and meshes at the initial time on the right. Recall that the representation of interfaces is performed thanks to the isolines of the function defined by (5.1).

Figure 5 shows the evolution of the interface position. The system tends to a position which minimizes the length of the interface while conserving the volume of phases, that is a circular interface. Note that the actual steady state is not yet achieved at the end of our computation $(t=4.8)$.

Figure 6 presents the convergence study. The reference solution is computed using the SImpl.(0.5) scheme with $\Delta t_{\text {ref }}=5 \times 10^{-4}$. Several computations are performed using the different schemes and for each of the following time steps $\Delta t_{j}: 10^{-1}, 5 \times 10^{-2}, 10^{-2}, 5 \times 10^{-3}, 10^{-3}$. The $\mathrm{L}^{2}$-norm of the corresponding errors $e_{j}(t)$ at time $t=3.8$ are represented in the picture on the left and convergence rates are presented in the table on the right. We essentially obtain the same results as in one dimension, that is a first order convergence for the Impl., CC., SImpl. schemes and a (almost) second order convergence for the SImpl.(0.5) scheme. The CC. scheme is still less accurate than the other ones.

\subsubsection{Ellipsoidal bubble - Dirichlet boundary conditions}

This experiment is performed on the space domain $[-0.1,0.1] \times[0,0.2]$ with the following parameters: the interface thickness $\varepsilon=6 \times 10^{-3}$, a constant mobility $M=10^{-4}$ and a surface tension between the two present 


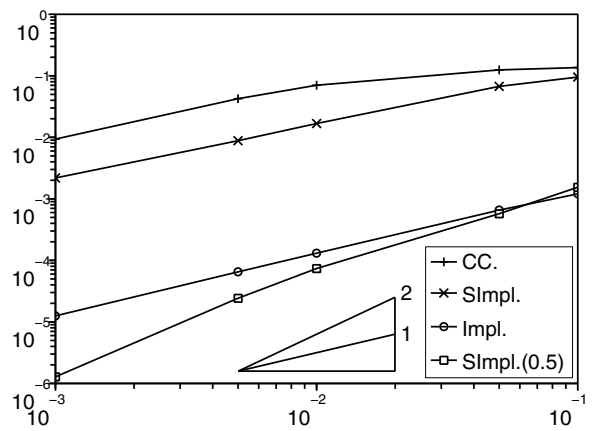

\begin{tabular}{||c|c||c|c|c|c||}
\hline \multirow{2}{*}{$j$} & \multirow{2}{*}{$\Delta t_{j}$} & \multicolumn{4}{c||}{$\frac{\ln \left(e_{j+1} / e_{j}\right)}{\ln \left(\Delta t_{j+1} / \Delta t_{j}\right)}$} \\
\cline { 3 - 6 } & & CC. & SImpl. & SImpl.(0.5) & Impl. \\
\hline \hline 1 & $10^{-1}$ & 0.1 & 0.5 & 1.4 & 0.9 \\
\hline 2 & $5 \times 10^{-2}$ & 0.4 & 0.9 & 1.3 & 1.0 \\
\hline 3 & $10^{-2}$ & 0.7 & 0.9 & 1.6 & 1.0 \\
\hline 4 & $5 \times 10^{-3}$ & 0.9 & 0.9 & 1.8 & 1.0 \\
\hline 5 & $10^{-3}$ & - & - & - & - \\
\hline
\end{tabular}

FiguRE 6. Errors $e_{j}(t)=\left|\mathbf{c}^{\Delta t_{j}}(t, \cdot)-\mathbf{c}^{\Delta t_{\text {ref }}}(t, \cdot)\right|_{\left(\mathrm{L}^{2}(\Omega)\right)^{3}}$ at time $t=3.8$ as a function of the time step $\Delta t_{j}$ (left) and convergence rates (right) obtained for the different schemes.
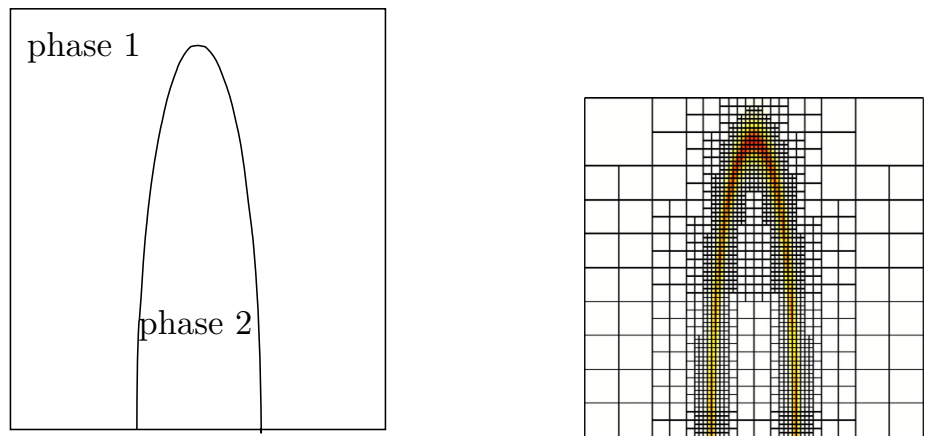

Figure 7. Configuration of test case (left) and initial position of interface (right). (Figure in color available online at http://www . esaim-m2an.org/.)

phases $\sigma=1$. The initial data is given by:

$$
c_{1}^{0}(x, y)=\frac{1}{2}\left[1+\tanh \left(\frac{2}{\varepsilon}\left(4 x^{2}+\frac{y^{2}}{12.25}\right)^{\frac{1}{2}}-0.05\right)\right], \quad c_{2}^{0}(x, y)=0,
$$

for all $(x, y) \in[-0.1,0.1] \times[0,0.2]$.

Figure 7 shows the initial configuration on the left and the position of the interfaces and meshes at the initial time on the right. We impose Neumann boundary conditions for both order parameters and chemical potentials except for the bottom part of the domain, that is $[-0.1,0.1] \times\{0\}$, where Dirichlet boundary conditions for the order parameters are imposed. Recall that the representation of interfaces is performed thanks to the isolines of the function defined by (5.1).

Figure 8 shows the evolution of the interface position. The system tends to a position which minimizes the length of the interface while conserving the volume of phases, the interface describes an arc of a circle since the value of the order parameter is imposed on the bottom part of the domain.

Figure 9 presents the convergence study. The reference solution is computed using the SImpl.(0.5) scheme with $\Delta t_{\text {ref }}=10^{-5}$. Several computations are performed using the different schemes and for each of the following time steps $\Delta t_{j}: 5 \times 10^{-3}, 10^{-3}, 5 \times 10^{-4}, 2 \times 10^{-4}, 10^{-4}, 5 \times 10^{-5}$. The $\mathrm{L}^{2}$-norm of the corresponding errors $e_{j}(t)$ at time $t=1.5$ are represented in the picture on the left and convergence rates are presented in the table on the right. We obtain a first order convergence for CC., Impl. and SImpl. schemes and a second order convergence 


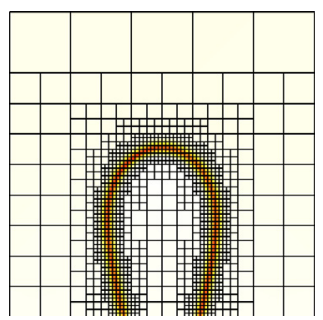

$t=0.5$

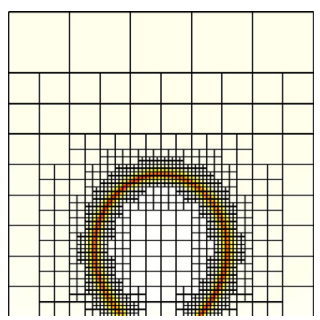

$t=1$.

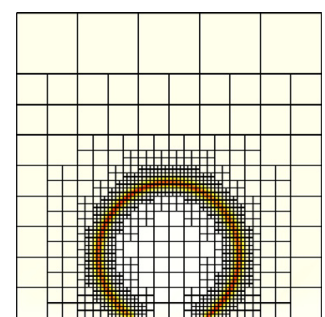

$t=1.5$

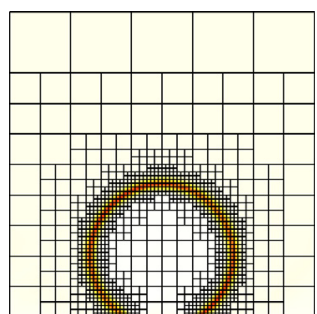

$t=5$.

Figure 8. Evolution of the interface position using the Impl. scheme with $\Delta t=5 \times 10^{-5}$.

(Figure in color available online at http://www.esaim-m2an.org/.)

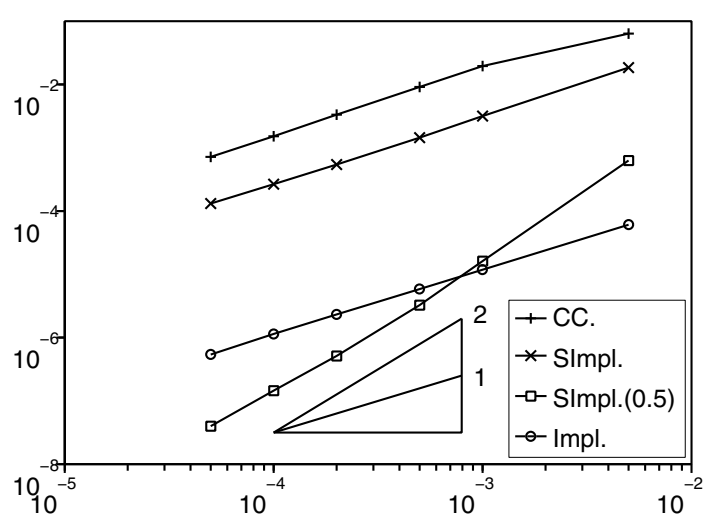

\begin{tabular}{||c|c||c|c|c|c||}
\hline \multirow{2}{*}{$j$} & \multicolumn{1}{|c||}{$\Delta t_{j}$} & \multicolumn{4}{|c||}{$\frac{\ln \left(e_{j+1} / e_{j}\right)}{\ln \left(\Delta t_{j+1} / \Delta t_{j}\right)}$} \\
\cline { 3 - 6 } & & CC. & SImpl. & SImpl.(0.5) & Impl. \\
\hline \hline 1 & $5 \times 10^{-3}$ & 0.7 & 1.1 & 2.3 & 1.0 \\
\hline 2 & $10^{-3}$ & 1.1 & 1.1 & 2.3 & 1.0 \\
\hline 3 & $5 \times 10^{-4}$ & 1.1 & 1.1 & 2.0 & 1.0 \\
\hline 4 & $2 \times 10^{-4}$ & 1.1 & 1.0 & 1.8 & 1.0 \\
\hline 5 & $10^{-4}$ & 1.1 & 1.0 & 1.9 & 1.1 \\
\hline 6 & $5 \times 10^{-5}$ & - & - & - & - \\
\hline
\end{tabular}

Figure 9. Errors $e_{j}(t)=\left|\mathbf{c}^{\Delta t_{j}}(t, \cdot)-\mathbf{c}^{\Delta t_{\text {ref }}}(t, \cdot)\right|_{\left(\mathrm{L}^{2}(\Omega)\right)^{3}}$ at time $t=1.5$ as a function of the time step $\Delta t_{j}$ (left) and convergence rates (right) obtained for the different schemes.

TABLE 2. Parameters values for the three phase test case in partial spreading situation.

\begin{tabular}{|c|c|c|c|c|c|c|c|c|c|}
\hline$\Omega$ & $\varepsilon$ & $M_{0}$ & $\sigma_{12}$ & $\sigma_{13}$ & $\sigma_{23}$ & $\Sigma_{1}$ & $\Sigma_{2}$ & $\Sigma_{3}$ & $\Lambda$ \\
\hline$[-0.3 ; 0.3] \times[-0.15 ; 0.15]$ & $10^{-2}$ & $10^{-4}$ & 1 & 0.8 & 1.4 & 0.4 & 1.6 & 1.2 & 0 \\
\hline
\end{tabular}

for the SImpl.(0.5) scheme. Remark that SImpl.(0.5) and Impl. schemes give significantly more accurate results than the CC. one.

\subsection{Three phase test cases}

In this section, we illustrate the properties of the different schemes with the spreading of a liquid lens between two stratified phases in two dimensions. In the following test cases, the initial solution is less smooth than it was in the previous two-phase test cases. Hence, we avoid to take the value 0.5 for the parameter $\beta$, since this value corresponds to the limit of unconditional stability of the Crank-Nicolson time stepping method for this problem. Moreover, for the same reason, we use the value $\beta=1$ (that is the implicit discretization of the diffusion term) for the first iteration even for the SImpl. $(\beta)$ scheme.

\subsubsection{Partial spreading situation}

The values of parameters are given in Table 2 . Note that in this case, all of the $\Sigma_{i}, i=1,2,3$, are positive. Hence, we take $\Lambda=0$ (see Sect. 3.6), so that the Cahn-Hilliard potential is $F=F_{0}$. 

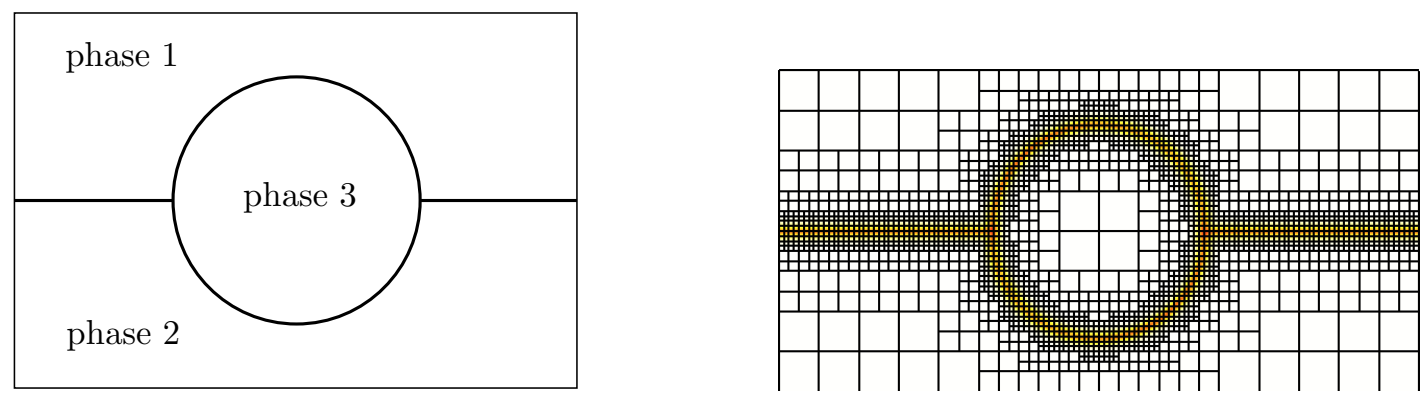

Figure 10. Configuration of test case (left) and initial position of interface (right). (Figure in color available online at http://www. esaim-m2an.org/.)

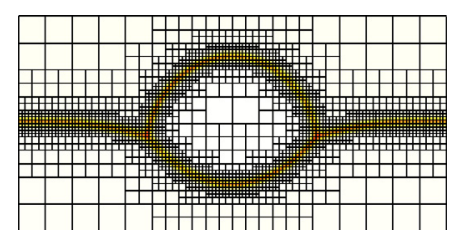

$t=0.2$

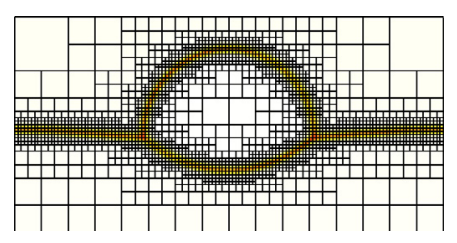

$t=2$.

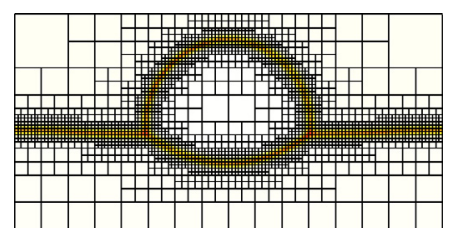

$t=5$.

Figure 11. Evolution of the interface position for $\Delta t=10^{-4}$ using the Impl. scheme. (Figure in color available online at http://www. esaim-m2an.org/.)

$$
\frac{\sin \theta_{1}}{\sigma_{23}}=\frac{\sin \theta_{2}}{\sigma_{13}}=\frac{\sin \theta_{3}}{\sigma_{12}}
$$

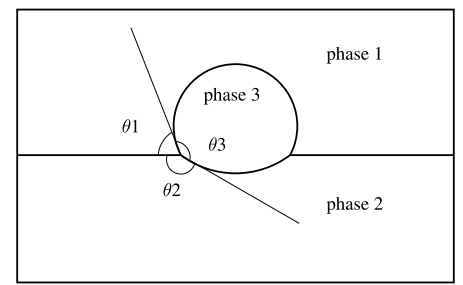

FIgURE 12. Theoretical shape of the lens at the equilibrium.

The initial data $\mathbf{c}^{0}$ is given by

$$
\begin{aligned}
c_{1}^{0}(\boldsymbol{x}) & =\frac{1}{2}\left[1+\tanh \left(\frac{2}{\varepsilon} \min (|\boldsymbol{x}|-0.1, y)\right)\right], \\
c_{2}^{0}(\boldsymbol{x}) & =\frac{1}{2}\left[1-\tanh \left(\frac{2}{\varepsilon} \max (-|\boldsymbol{x}|+0.1, y)\right)\right], \\
c_{3}^{0}(\boldsymbol{x}) & =1-c_{1}(\boldsymbol{x})-c_{2}(\boldsymbol{x}),
\end{aligned}
$$

where $\boldsymbol{x}=(x, y) \in \Omega$. This corresponds (Fig. 10) to an initial spherical captive bubble of phase 3 between the two stratified phases 1 and 2. Recall that the representation of interfaces is performed thanks to the function defined by (5.1).

Figure 11 shows the evolution of the interface position. At equilibrium, the expected shape of the lens is the intersection of two spherical caps whose contact angles depend on the three surface tensions through the Young relations, as shown in Figure 12.

Figure 13 presents the convergence study. The reference solution is computed using the Impl. scheme with $\Delta t_{\text {ref }}=10^{-4}$. Several computations are performed using the different schemes and for each of the following 


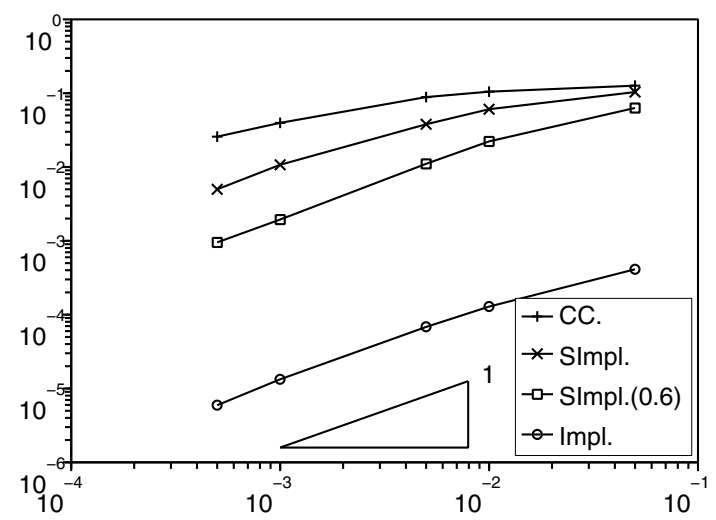

\begin{tabular}{||c|c||c|c|c|c||}
\hline \multirow{2}{*}{$j$} & \multicolumn{2}{|c||}{$\Delta t_{j}$} & \multicolumn{4}{|c||}{$\frac{\ln \left(e_{j+1} / e_{j}\right)}{\ln \left(\Delta t_{j+1} / \Delta t_{j}\right)}$} \\
\cline { 3 - 6 } & & CC. & SImpl. & SImpl.(0.6) & Impl. \\
\hline \hline 1 & $5 \times 10^{-2}$ & 0.1 & 0.3 & 0.6 & 0.7 \\
\hline 2 & $10^{-2}$ & 0.2 & 0.7 & 1.0 & 0.9 \\
\hline 3 & $5 \times 10^{-3}$ & 0.5 & 0.8 & 1.1 & 1.0 \\
\hline 4 & $10^{-3}$ & 0.6 & 1.1 & 1.0 & 1.2 \\
\hline 5 & $5 \times 10^{-4}$ & - & - & - & - \\
\hline
\end{tabular}

Figure 13. Errors $e_{j}(t)=\left|\mathbf{c}^{\Delta t_{j}}(t, \cdot)-\mathbf{c}^{\Delta t_{\mathrm{ref}}(t, \cdot)}\right|_{\left(\mathrm{L}^{2}(\Omega)\right)^{3}}$ at time $t=2$. as a function of the time step $\Delta t_{j}$ (left) and convergence rates (right) obtained for the different schemes.

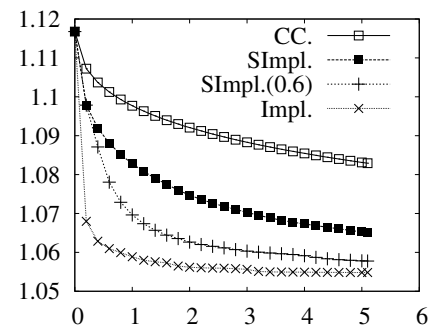

(a) $\Delta t=10^{-1}$

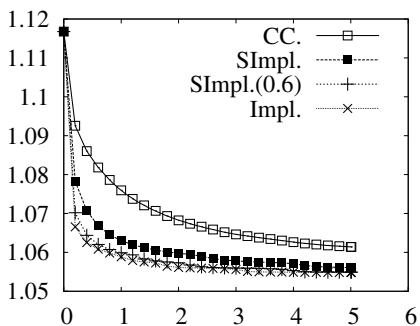

(b) $\Delta t=10^{-2}$

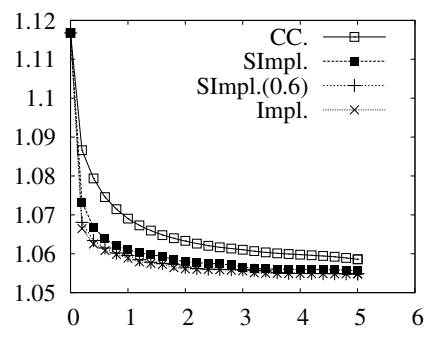

(c) $\Delta t=5 \times 10^{-3}$

FiguRE 14. Time evolution of energy in a partial spreading situation.

time steps $\Delta t_{j}: 5 \times 10^{-2}, 10^{-2}, 5 \times 10^{-3}, 10^{-3}, 5 \times 10^{-4}$. The $\mathrm{L}^{2}$-norm of the corresponding errors $e_{j}(t)$ at time $t=2$. are represented in the picture on the left and the convergence rates are presented in the table on the right. As expected, we obtain a first order convergence for the four schemes. Nevertheless, the Impl. scheme is clearly the more accurate. We observe in particular a three order of magnitude ratio in the error compared to the CC. scheme.

In Figure 14, we show the discrete energy $\mathcal{F}_{\Sigma, \varepsilon}^{\text {triph }}\left(\mathbf{c}_{h}^{n}\right)$ as a function of time $t_{n} \in\left[0, t_{f}\right]$. For each of the four schemes, we performed three simulations with $\Delta t=10^{-1}, 10^{-2}$ and $5 \times 10^{-3}$. Figure 14 shows a comparison between the four schemes, using the same time step. SImpl. and SImpl.(0.6) schemes give significantly more accurate results than the CC. one.

Figure 15 shows the influence of the schemes on the bubble shape at the time $t=2$. With the Impl. scheme, the same shape is obtained for the three time steps. For large time steps, the CC. scheme does not give the bubble shape which is expected. This phenomenon is significantly reduced by the use of the SImpl. or SImpl.(0.6) schemes.

Remark 5.1. In the computations presented in this section, the total number of degrees of freedom is $\sim 8500$ (the actual value varies a little at each iteration due to the adaptation procedure). To achieve the same accuracy (that is the same mesh size inside the interfaces) with a uniform grid, we should have used 33540 degrees of freedom. This shows the importance of using an adaptive refinement method for such models.

The case $\beta=\frac{1}{2}$ is a limit stability case in the analysis proposed above since most of our results hold only for $\beta>\frac{1}{2}$. We illustrate here that instabilities can actually occur for $\beta=\frac{1}{2}$. In Figure 16, we compare the results for the same set of physical parameters $\left(\sigma_{12}=\sigma_{13}=0.07\right.$ and $\left.\sigma_{23}=0.05\right)$ and the same value of the time step 

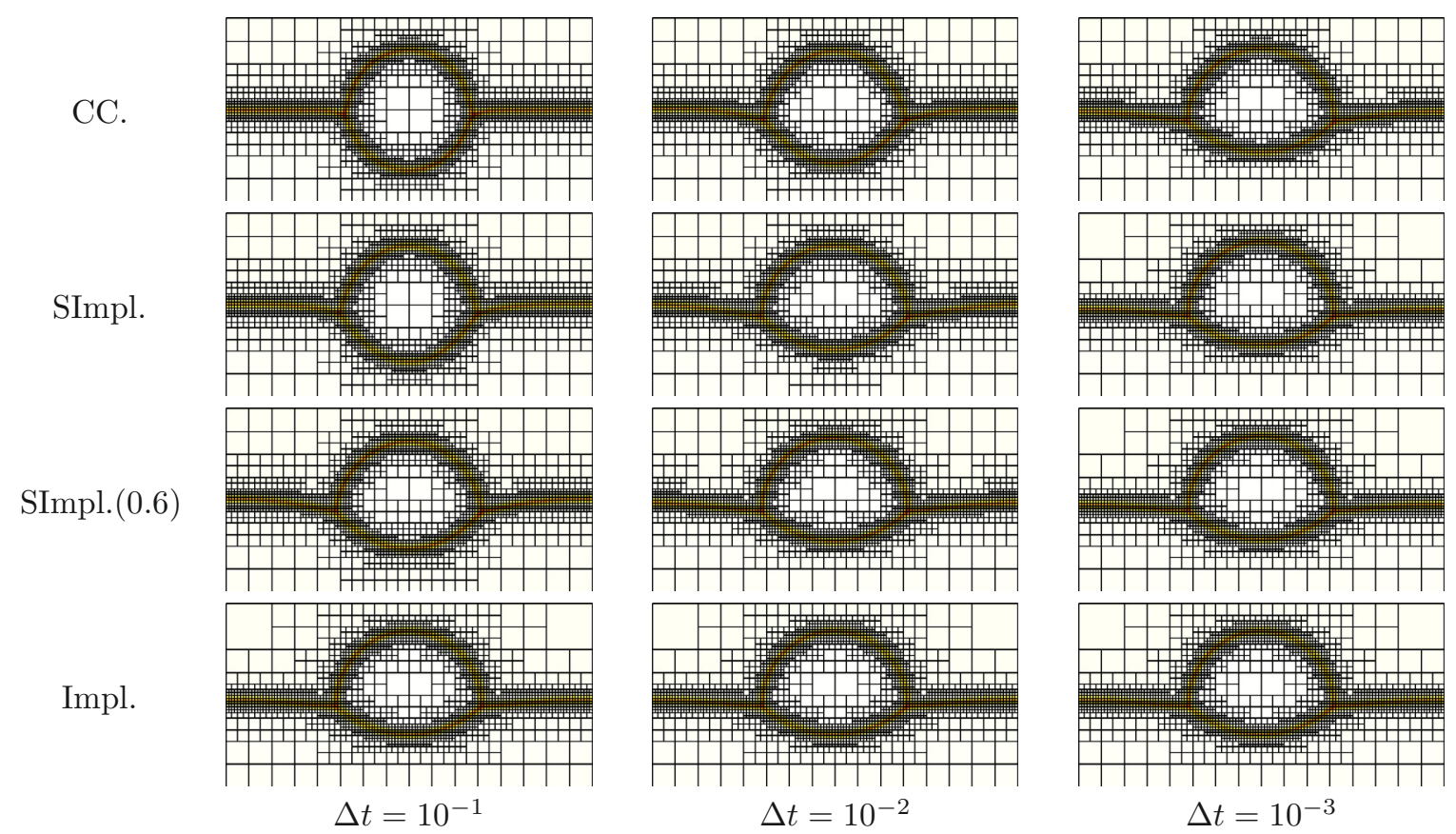

$$
\Delta t=10^{-3}
$$

FiguRE 15. Influence of schemes on bubble shape at $t=2$. (Figure in color available online at http://www.esaim-m2an.org/.)

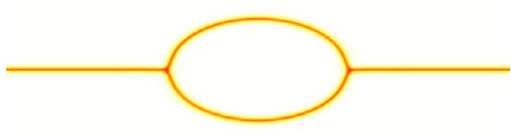

Implicit Euler : $\beta=1$

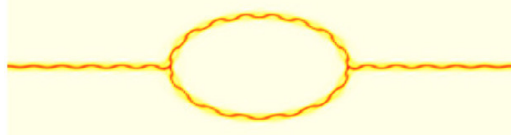

Crank-Nicolson : $\beta=\frac{1}{2}$

FiguRE 16. Influence of the parameter $\beta$. (Figure in color available online at http://www . esaim-m2an.org/.)

TABLE 3. Parameters values for the three phase test case in partial spreading situation.

\begin{tabular}{|c|c|c|c|c|c|c|c|c|c|}
\hline$\Omega$ & $\varepsilon$ & $M_{0}$ & $\sigma_{12}$ & $\sigma_{13}$ & $\sigma_{23}$ & $\Sigma_{1}$ & $\Sigma_{2}$ & $\Sigma_{3}$ & $\Lambda$ \\
\hline$[-0.3 ; 0.3] \times[-0.3 ; 0.2]$ & $10^{-2}$ & $10^{-4}$ & 1 & 1 & 3 & -1 & 3 & 3 & $7 / 3$ \\
\hline
\end{tabular}

$\left(\Delta t=10^{-3}\right)$. We show the position of the interface at the same computational time $t=3 \times 10^{-3}$. We observe interface instabilities in the case $\beta=\frac{1}{2}$ which can be also observed in Figure 17 where we show the evolution of the energy during the computation. As predicted by Proposition 3.2, the energy decreases for $\beta=1$ but does not remain bounded for $\beta=\frac{1}{2}$. Indeed, note that in this last case the limit time step $\Delta t_{0}$ given in this proposition vanishes.

\subsubsection{Total spreading situation}

The values of parameters are given in Table 3 . 


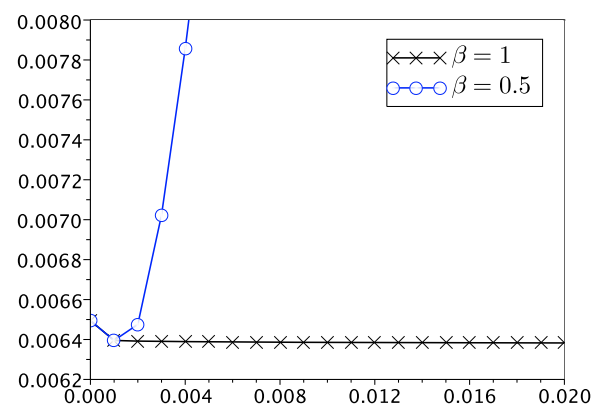

Figure 17. Evolution in time of the energy for $\beta=\frac{1}{2}$ and $\beta=1$.
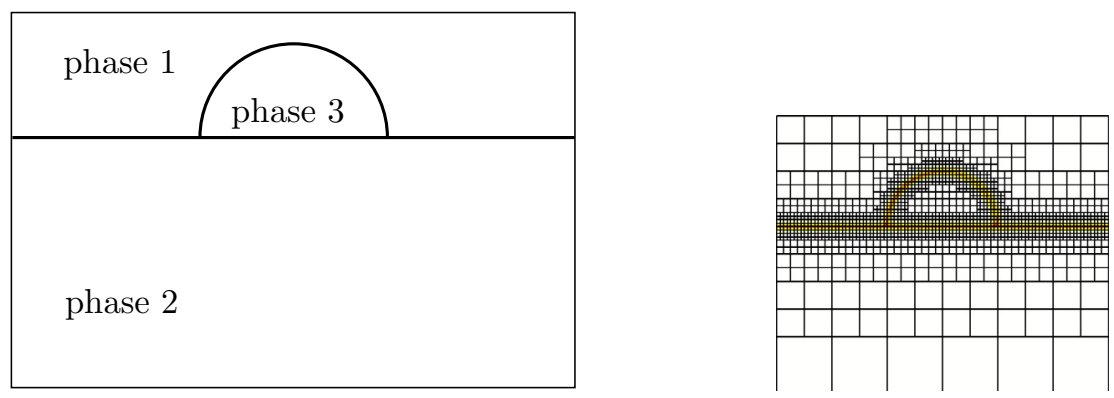

Figure 18. Configuration of test case (left) and initial position of interface (right). (Figure in color available online at http://www.esaim-m2an.org/.)

The initial data $\mathbf{c}^{0}$ is given by

$$
\begin{aligned}
c_{1}^{0}(\boldsymbol{x}) & =\frac{1}{2}\left[1+\tanh \left(\frac{2}{\varepsilon} \min \left(\sqrt{x^{2}+y^{2}}-0.1, y\right)\right)\right] \\
c_{2}^{0}(\boldsymbol{x}) & =\frac{1}{2}\left[1-\tanh \left(\frac{2}{\varepsilon} y\right)\right], \\
c_{3}^{0}(\boldsymbol{x}) & =1-c_{1}(\boldsymbol{x})-c_{2}(\boldsymbol{x}),
\end{aligned}
$$

where $\boldsymbol{x}=(x, y) \in \Omega$.

This corresponds (Fig. 18) to an initial bubble of phase 3 put on the interface between the two stratified phases 1 and 2 .

In this case, $\Sigma_{1}$ is negative but condition (1.5) holds. It corresponds to the case of the extraction of the bubble (Fig. 19): at the steady state the bubble is entirely within one of the other phases. We have to take $\Lambda$ large enough to ensure the positivity of the Cahn-Hilliard potential $F$ (see Sect. 3.6). We take here $\Lambda=7 / 3$.

Figure 20 shows that the corresponding potential $F$ has the expected shape: $F$ is non negative and has only three minima which correspond to pure phases. The potential $F$ is represented on the hyperplane $\mathcal{S}$ using barycentric coordinates.

We perform simulations using the different schemes with time steps $\Delta t: 10^{-1}, 5 \times 10^{-2}, 10^{-2}, 5 \times 10^{-3}$, $10^{-3}, 5 \times 10^{-4}, 10^{-4}$. We observe that the Newton linearization method fails to converge when using the Impl. scheme unless the time step is smaller than $10^{-4}$. Table 4 shows the maximum of the number of iterations in the Newton linearization method over all the time iterations of the simulation. The CC. scheme appears as the more robust one because the computation nicely runs for any value of the time step. SImpl. and SImpl.(0.6) schemes work for a large range of time steps. 


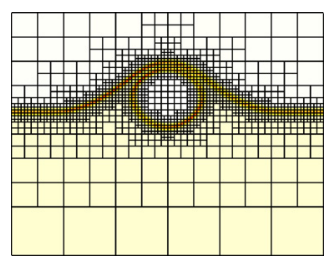

$t=2$.

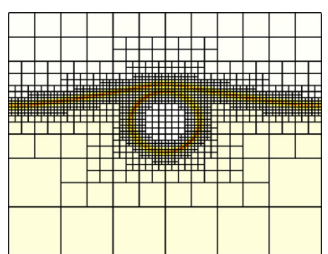

$t=30$.

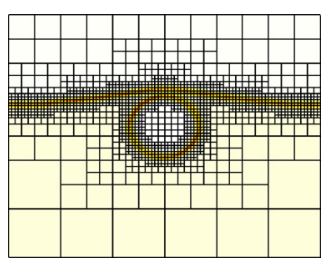

$t=300$.

FIgure 19. Evolution of the interface position for $\Delta t=10^{-3}$ using the SImpl. scheme. (Figure in color available online at http: //www.esaim-m2an.org/.)
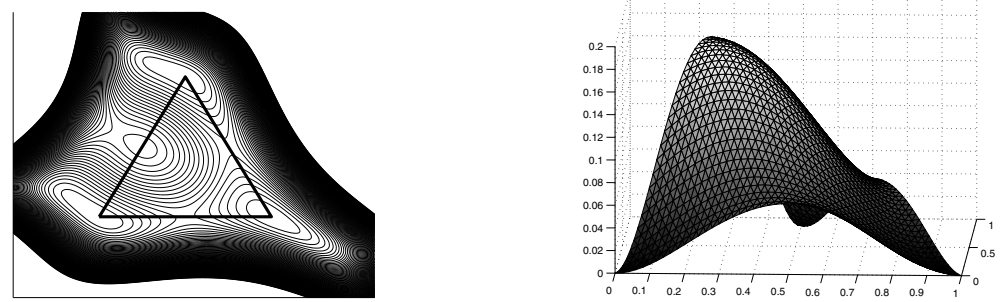

Figure 20. Cahn-Hilliard potential $F$ using barycentric coordinates.

TABLE 4. Number of iterations in the Newton linearization method. The symbol "_" means that there is no convergence.

\begin{tabular}{|c||c|c|c|c|c|c|c|}
\hline Scheme & $10^{-1}$ & $5 \times 10^{-2}$ & $10^{-2}$ & $5 \times 10^{-3}$ & $10^{-3}$ & $5 \times 10^{-4}$ & $10^{-4}$ \\
\hline \hline CC. & 5 & 5 & 5 & 5 & 5 & 5 & 4 \\
\hline SImpl. & - & - & 9 & 9 & 6 & 6 & 5 \\
\hline SImpl.(0.6) & - & - & 29 & - & 7 & 6 & 5 \\
\hline Impl. & - & - & - & - & - & - & 7 \\
\hline \hline CPU time & 5 min & 9 min & 40 min & $1 \mathrm{~h} 10$ & $5 \mathrm{~h} 45$ & $11 \mathrm{~h}$ & $53 \mathrm{~h}$ \\
\hline
\end{tabular}

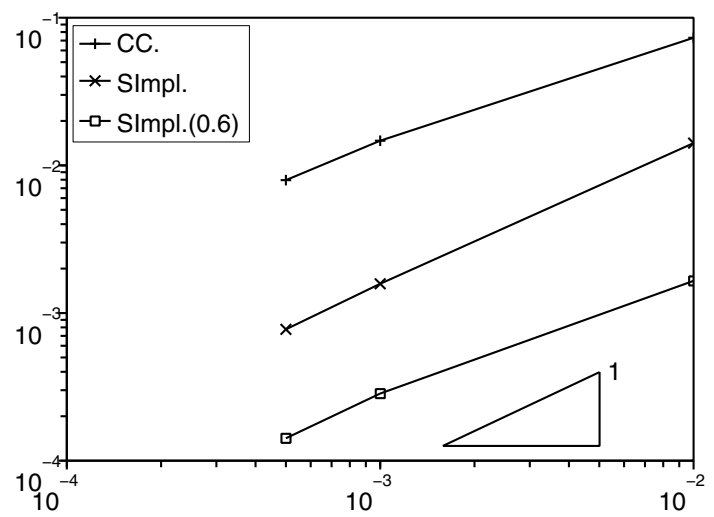

\begin{tabular}{||c|c||c|c|c||}
\hline \multirow{2}{*}{$j$} & \multicolumn{1}{|c||}{$\Delta t_{j}$} & \multicolumn{3}{c||}{$\frac{\ln \left(e_{j+1} / e_{j}\right)}{\ln \left(\Delta t_{j+1} / \Delta t_{j}\right)}$} \\
\cline { 3 - 5 } & & CC. & SImpl. & SImpl.(0.6) \\
\hline \hline 1 & $10^{-2}$ & 0.7 & 1.0 & 0.8 \\
\hline 2 & $10^{-3}$ & 0.9 & 1.0 & 1.0 \\
\hline 3 & $5 \times 10^{-4}$ & - & - & - \\
\hline
\end{tabular}

Figure 21. Errors $e_{j}(t)=\left|\mathbf{c}^{\Delta t_{j}}(t, \cdot)-\mathbf{c}^{\Delta t_{\mathrm{ref}}}(t, \cdot)\right|_{\left(\mathrm{L}^{2}(\Omega)\right)^{3}}$ at time $t=3.8$ as a function of the time step $\Delta t_{j}$ (left) and convergence rates (right) obtained for the different schemes. 


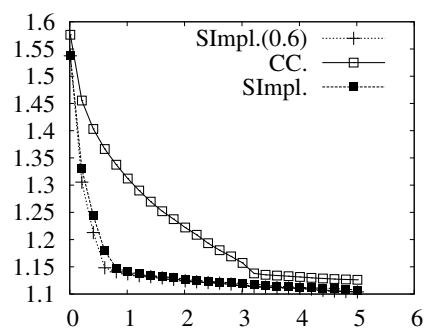

(a) $\Delta t=10^{-2}$

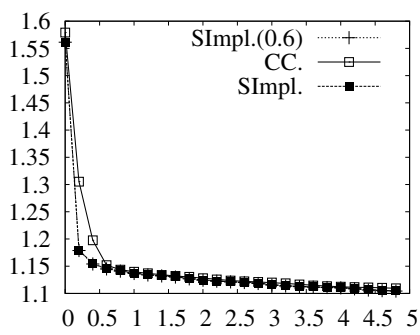

(b) $\Delta t=10^{-3}$

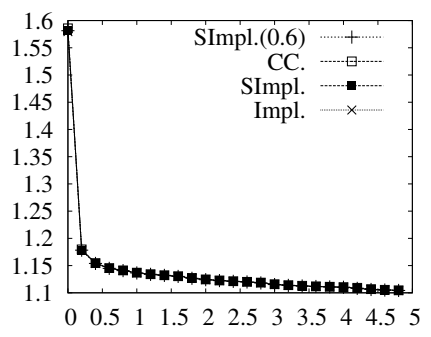

(c) $\Delta t=10^{-4}$

FiguRE 22. Time evolution of energy in a partial spreading situation.

These results have to be balanced by the convergence rates presented in Figure 21. Indeed, The CC. scheme appears to be the less accurate compared to the SImpl. and SImpl.(0.6) ones, even though the three schemes are first order convergent. We can also visualize the difference between schemes thanks to Figure 22 which shows how the discrete energy decrease when using the different schemes. We performed simulations for $\Delta t=10^{-2}$, $10^{-3}, 10^{-4}$ and we observed that SImpl. and SImpl.(0.6) schemes give significantly more accurate results than the CC. one.

\section{Conclusion}

We proposed here a full discretization of the ternary Cahn-Hilliard model taken from [8]. Different time discretizations are compared with the objective to get an accurate and robust algorithm for a wide range of situations including partial and total spreading situations.

At the theoretical level, for the implicit scheme, we are able to show the convergence of the discrete solution only in the case of partial spreading situation. Moreover, even in these situations, the Impl. scheme ensures the decrease of discrete energy only for small enough time steps. Convex-concave and semi-implicit schemes enable to show the convergence even for total spreading cases (provided that the condition (1.5) holds) and ensure the decrease of the discrete energy for all time steps. In practice, for partial spreading situation, the implicit scheme is the more accurate and the semi-implicit one enables to reduce the truncation error compared with the convex-concave one. For total spreading situations, we observe in some numerical computations that the implicit scheme can be ill-posed if the time step is not small enough whereas we can prove that the semiimplicit scheme is well-posed. Using the implicit scheme requires smaller time step, thus leading to a much higher computational cost.

In summary, we can say that the semi-implicit discretization of the non linear terms we proposed is a good compromise between robustness and accuracy, compared to the other more classical possible discretizations.

Acknowledgements. The authors want to express their gratitude to Celine Lapuerta and Bruno Piar for their support during the preparation of this work and to thank the referees for their careful reading of the paper and their valuable remarks.

\section{REFERENCES}

[1] J.W. Barrett and J.F. Blowey, An improved error bound for a finite element approximation of a model for phase separation of a multi-component alloy. IMA J. Numer. Anal. 19 (1999) 147-168.

[2] J.W. Barrett and J.F. Blowey, Finite element approximation of an Allen-Cahn/Cahn-Hilliard system. IMA J. Numer. Anal. 22 (2002) 11-71.

[3] J.W. Barrett, J.F. Blowey and H. Garcke, Finite element approximation of the Cahn-Hilliard equation with degenerate mobility. SIAM J. Numer. Anal. 37 (1999) 286-318.

[4] J.W. Barrett, J.F. Blowey and H. Garcke, On fully practical finite element approximations of degenerate Cahn-Hilliard systems. ESAIM: M2AN 35 (2001) 713-748. 
[5] J.F. Blowey and C.M. Elliott, The Cahn-Hilliard gradient theory for phase separation with nonsmooth free energy. II. Numerical analysis. Eur. J. Appl. Math. 3 (1992) 147-179.

[6] J.F. Blowey, M.I.M. Copetti and C.M. Elliott, Numerical analysis of a model for phase separation of a multi-component alloy. IMA J. Numer. Anal. 16 (1996) 111-139.

[7] F. Boyer, A theoretical and numerical model for the study of incompressible mixture flows. Comput. Fluids 31 (2002) 41-68.

[8] F. Boyer and C. Lapuerta, Study of a three component Cahn-Hilliard flow model. ESAIM: M2AN 40 (2006) 653-687.

[9] F. Boyer, C. Lapuerta, S. Minjeaud and B. Piar, A local adaptive refinement method with multigrid preconditioning illustrated by multiphase flows simulations, in CANUM 2008, ESAIM Proc. 27, EDP Sciences, Les Ulis (2009) 15-53.

[10] F. Boyer, C. Lapuerta, S. Minjeaud, B. Piar and M. Quintard, Cahn-Hilliard/Navier-Stokes model for the simulation of three-phase flows. Transp. Porous Media 82 (2010) 463-483.

[11] K. Deimling, Nonlinear functional analysis. Springer-Verlag (1985).

[12] Q. Du and R.A. Nicolaides, Numerical analysis of a continuum model of phase transition. SIAM J. Numer. Anal. 28 (1991) $1310-1322$.

[13] C.M. Elliott, The Cahn-Hilliard model for the kinetics of phase separation, in Mathematical models for phase change problems, Óbidos, 1988, Internat. Ser. Numer. Math. 88, Birkhäuser, Basel (1989) 35-73.

[14] C.M. Elliott and H. Garcke, Diffusional phase transitions in multicomponent systems with a concentration dependent mobility matrix. Physica D 109 (1997) 242-256.

[15] C.M. Elliott and S. Luckhaus, A generalised diffusion equation for phase separation of a multi-component mixture with interfacial free energy. IMA Preprint Series \# 887 (1991).

[16] C.M. Elliott and A.M. Stuart, The global dynamics of discrete semilinear parabolic equations. SIAM J. Numer. Anal. 30 (1993) 1622-1663.

[17] A. Ern and J.-L. Guermond, Theory and Pratice of Finite Elements, Applied Mathematical Sciences 159. Springer (2004).

[18] D.J. Eyre, Unconditionally gradient stable time marching the Cahn-Hilliard equation, in Computational and mathematical models of microstructural evolution, San Francisco, CA, 1998, Mater. Res. Soc. Sympos. Proc. 529, MRS, Warrendale, PA (1998) 39-46.

[19] X. Feng, Fully discrete finite element approximations of the Navier-Stokes-Cahn-Hilliard diffuse interface model for two-phase fluid flows. SIAM J. Numer. Anal. 44 (2006) 1049-1072.

[20] X. Feng, Y. He and C. Liu, Analysis of finite element approximations of a phase field model for two-phase fluids. Math. Comp. 76 (2007) 539-571.

[21] H. Garcke and B. Stinner, Second order phase field asymptotics for multi-component systems. Interface Free Boundaries 8 (2006) 131-157.

[22] H. Garcke, B. Nestler and B. Stoth, A multiphase field concept: numerical simulations of moving phase boundaries and multiple junctions. SIAM J. Appl. Math. 60 (2000) 295-315.

[23] J. Kim and J. Lowengrub, Phase field modeling and simulation of three-phase flows. Interfaces Free Boundaries 7 (2005) $435-466$.

[24] J. Kim, K. Kang and J. Lowengrub, Conservative multigrid methods for Cahn-Hilliard fluids. J. Comput. Phys. 193 (2004) $511-543$.

[25] J. Kim, K. Kang and J. Lowengrub, Conservative multigrid methods for ternary Cahn-Hilliard systems. Commun. Math. Sci. 2 (2004) 53-77.

[26] C. Lapuerta, Échanges de masse et de chaleur entre deux phases liquides stratifiées dans un écoulement à bulles. Mathématiques appliquées, Université de Provence, France (2006).

[27] H.G. Lee and J. Kim, A second-order accurate non-linear difference scheme for the n-component Cahn-Hilliard system. Physica A 387 (2008) 4787-4799.

[28] B. Nestler, H. Garcke and B. Stinner, Multicomponent alloy solidification: Phase-field modeling and simulations. Phys. Rev. E 71 (2005) 041609.

[29] PELICANS, Collaborative Development environment, https://gforge.irsn.fr/gf/project/pelicans/.

[30] J.S. Rowlinson and B. Widom, Molecular theory of capillarity. Clarendon Press (1982).

[31] J.M. Seiler and K. Froment, Material effects on multiphase phenomena in late phases of severe accidents of nuclear reactors. Multiph. Sci. Technol. 12 (2000) 117-257.

[32] J. Simon, Compact sets in the space $L^{p}(0, T ; B)$. Ann. Mat. Pura Appl. 146 (1987) 65-96. 